quatrième série-tome 45 fascicule 6 novembre-décembre 2012

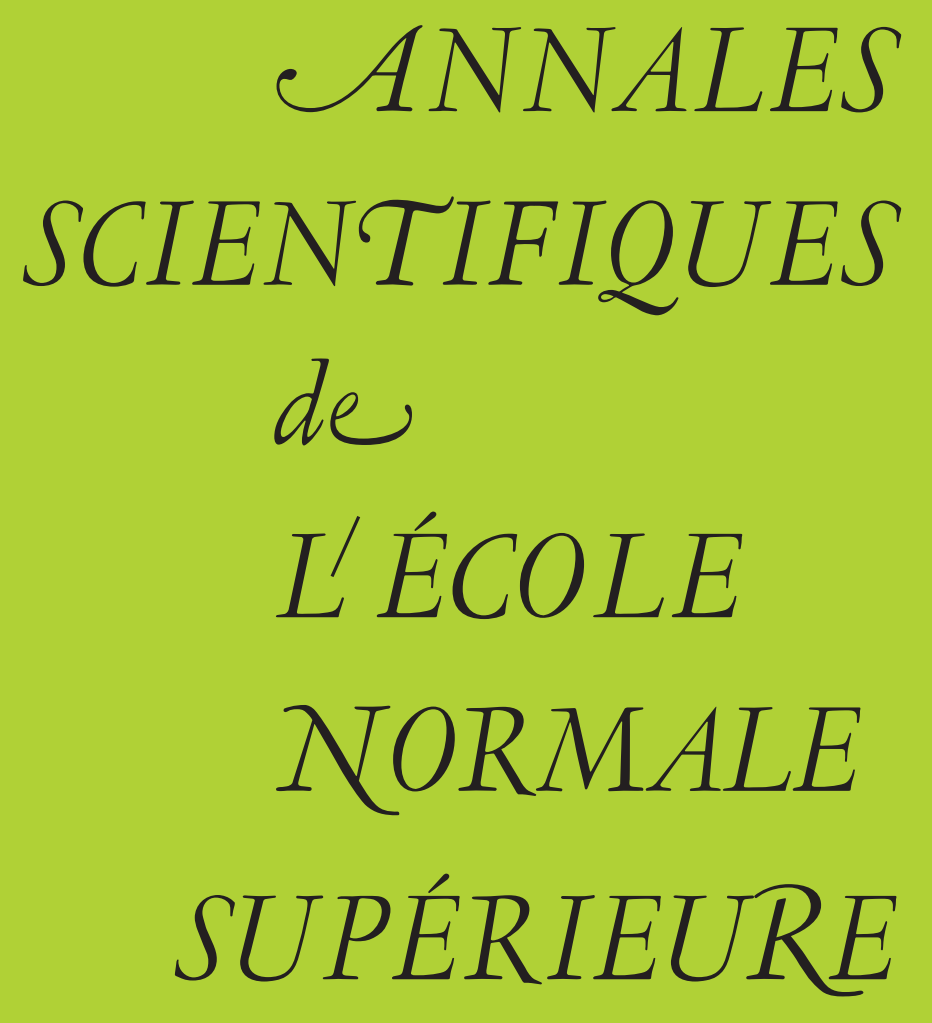

Viviane BALADI \& Daniel SMANIA

Linear response for smooth deformations of generic nonuniformly byperbolic unimodal maps 


\title{
LINEAR RESPONSE FOR SMOOTH DEFORMATIONS OF GENERIC NONUNIFORMLY HYPERBOLIC UNIMODAL MAPS
}

\author{
BY Viviane BALADI and Daniel SMANIA
}

\begin{abstract}
We consider $C^{2}$ families $t \mapsto f_{t}$ of $C^{4}$ unimodal maps $f_{t}$ whose critical point is slowly recurrent, and we show that the unique absolutely continuous invariant measure $\mu_{t}$ of $f_{t}$ depends differentiably on $t$, as a distribution of order 1 . The proof uses transfer operators on towers whose level boundaries are mollified via smooth cutoff functions, in order to avoid artificial discontinuities. We give a new representation of $\mu_{t}$ for a Benedicks-Carleson map $f_{t}$, in terms of a single smooth function and the inverse branches of $f_{t}$ along the postcritical orbit. Along the way, we prove that the twisted cohomological equation $v=\alpha \circ f-f^{\prime} \alpha$ has a continuous solution $\alpha$, if $f$ is Benedicks-Carleson and $v$ is horizontal for $f$.

RÉsumé. - Nous considérons des familles $t \mapsto f_{t}$ d'applications unimodales $C^{4}$, de récurrence postcritique lente, avec une dépendance $C^{2}$ en fonction du paramètre $t$. Nous montrons que l'unique mesure invariante $\mu_{t}$ de $f_{t}$ est différentiable en fonction de $t$, en tant que distribution d'ordre 1 . La preuve utilise des opérateurs de transfert sur des tours dont les bords sont mollifiés avec des fonctions de troncation lisses, pour éviter l'introduction de discontinuités artificielles. Nous donnons de plus une représentation de $\mu_{t}$ dépendant d'une unique fonction lisse et des branches inverses de $f_{t}$ le long de l'orbite postcritique. Nous prouvons enfin que l'équation cohomologique tordue $v=\alpha \circ f-f^{\prime} \alpha$ admet une solution continue $\alpha$, si $f$ est Benedicks-Carleson et $v$ est horizontal pour $f$.
\end{abstract}

\section{Introduction}

The linear response problem for discrete-time dynamical systems can be posed in the following way. Suppose that for each parameter $t$ (or many parameters $t$ ) in a smooth family

\footnotetext{
We thank D. Ruelle for conversations and e-mails, D. Schnellmann for pointing out many typos in a previous version of this text, and the anonymous referees for helpful comments. This work was started while D. Smania was visiting the DMA of École Normale Supérieure in 2008-09 and finished while V. Baladi participated in a semester at Institut Mittag-Leffler (Djursholm, Sweden) in 2010. We gratefully acknowledge the hospitality of both institutions. V.B. is partially supported by GDRE GREFI-MEFI and ANRBLAN08-2_313375, DynNonHyp. D.S. is partially supported by FAPESP 2008/02841-4 and 2010/08654-1, CAPES 1364-08-1, CNPq 310964/2006-7 and 303669/2009-8.
} 
of maps $t \mapsto f_{t}$ with $f_{t}: M \rightarrow M$, ( $M$ a compact Riemann manifold, say) there exists a unique physical (or SRB) measure $\mu_{t}$. (See [63] for a discussion of SRB measures.) One can ask for conditions which ensure the differentiability, possibly in the sense of Whitney, of the function $\mu_{t}$ in a weak sense (in the weak $*$-topology, i.e., as a distribution of order 0 , or possibly as a distribution of higher order). Ruelle has discussed this problem in several survey papers [46], [48], [50], to which we refer for motivation.

The case of smooth hyperbolic dynamics has been settled over a decade ago ([25], [45]), although recent technical progress in the functional analytic tools (namely, the introduction of anisotropic Sobolev spaces on which the transfer operator has a spectral gap) has allowed for a great simplification of the proofs (see, e.g., [19]): For smooth Anosov diffeomorphisms $f_{s}$ and a $C^{1}$ observable $A$, letting

$$
X_{s}=\left.\partial_{t} f_{t}\right|_{t=s} \circ f_{s}^{-1}
$$

Ruelle [45], [47] obtained the following explicit linear response formula (the derivative here is in the usual sense)

$$
\left.\partial_{t} \int A d \mu_{t}\right|_{t=0}=\Psi_{A}(1),
$$

where $\Psi_{A}(z)$ is the susceptibility function

$$
\Psi_{A}(z)=\sum_{k=0}^{\infty} \int z^{k}\left\langle X_{0}, \operatorname{grad}\left(A \circ f_{0}^{k}\right)\right\rangle d \mu_{0},
$$

and the series $\Psi_{A}(z)$ at $z=1$ converges exponentially. In fact, in the Anosov case, the susceptibility function is holomorphic in a disc of radius larger than 1 . This is related to the fact (see [7] for a survey and references) that the transfer operator of each $f_{s}$ has a spectral gap on a space which contains not only the product of the distribution $\mu_{s}$ and the smooth vector field $X_{s}$, but also the derivative of that product, that is, $\left\langle X_{s}, \operatorname{grad} \mu_{s}\right\rangle+\left(\operatorname{div} X_{s}\right) \mu_{s}$.

One feature of smooth hyperbolic dynamics is structural stability: Each $f_{t}$, for small $t$, is topologically conjugated to $f_{0}$ via a homeomorphism $h_{t}$, which turns out to depend smoothly on the parameter $t$. With the exception of a deep result of Dolgopyat [21] on rapidly mixing partially hyperbolic systems (where structural stability may be violated, but where there are no critical points and shadowing holds for a set of points of large measure, so that the bifurcation structure is relatively mild), the study of linear response in the absence of structural stability, or in the presence of critical points, has begun only recently.

However, the easier property of continuity of $\mu_{t}$ with respect to $t$ (in other words, statistical stability) has been established also in the presence of critical points: For piecewise expanding unimodal interval maps, Keller [26] proved in 1982 that the density $\phi_{t}$ of $\mu_{t}$, viewed as an element of $L^{1}$, has a modulus of continuity at least $t \ln t$, so that $t \mapsto \phi_{t}$ is $r$-Hölder, for any exponent $r \in(0,1)$. For nonuniformly smooth unimodal maps, in general not all nearby maps $f_{t}$ admit an SRB measure even if $f_{0}$ does. Therefore, continuity of $t \mapsto \mu_{t}$ can only be proved in the sense of Whitney, on a set of "good" parameters. This was done by Tsujii [58] and Rychlik-Sorets [53] in the 90's. More recently, Alves et al. [2], [1] proved that for Hénon maps, $t \mapsto \mu_{t}$ is continuous in the sense of Whitney in the weak $*$-topology. (We refer, e.g., to [8] for more references.)

Differentiability of $\mu_{t}$, even in the sense of Whitney, is a more delicate issue, even in dimension one. For nonuniformly hyperbolic smooth unimodal maps $f_{t}$ with a quadratic critical 
point $\left(f_{t}^{\prime \prime}(c)<0\right)$, it is known [61], [30] that the density $\phi_{t}$ of the absolutely continuous invariant measure $\mu_{t}$ of $f_{t}$ has singularities (called spikes) of the form ${\sqrt{x-c_{k, t}}}^{-1}$, where the $c_{k, t}=f_{t}^{k}(c)$ are the points along the forward orbit of the critical point $c$. Thus, the derivative $\phi_{t}^{\prime}$ of the invariant density has nonintegrable singularities, and the transfer operator cannot have a spectral gap in general on a space containing $\left(X_{t} \phi_{t}\right)^{\prime}$. In fact, the radius of convergence of the susceptibility function $\Psi_{A}(z)$ is very likely strictly smaller than 1 in general. Ruelle [49] observed however that, in the case of a subhyperbolic (preperiodic) critical point for a real analytic unimodal map, $\Psi_{A}(z)$ is meromorphic in a disc of radius larger than 1 , and that 1 is not a pole of $\Psi_{A}(z)$. He expressed the hope that the value $\Psi_{A}(1)$ obtained by analytic continuation could correspond to the actual derivative of the SRB measure, at least in the sense of Whitney.

This analytic continuation phenomenon in the subhyperbolic smooth unimodal case (where a finite Markov partition exists) could well be a red herring, in view of the linear response theory for the "toy model" of piecewise expanding interval maps that we recently established in a series of papers [7], [10], [12], [13]: Unimodal piecewise expanding interval maps $f_{t}$ have a unique SRB measure, whose density $\phi_{t}$ is a function of bounded variation (since $\phi_{t}^{\prime}$ is a measure, the situation is much easier than for smooth unimodal maps). In [7], [10], [14], we showed that Keller's [26] $t \ln t$ modulus of continuity was optimal (see also [35]): In fact, there exist smooth families $f_{t}$ so that $t \mapsto \mu_{t}$ is not Lipschitz, even when viewed as a distribution of arbitrarily high order, and even in the sense of Whitney. Such counter-examples $f_{t}$ are transversal to the topological class of $f_{0}$. If, on the contrary, the family $f_{t}$ is tangent at $t=0$ to the topological class of $f_{0}$ (we say that $f_{t}$ is horizontal) then ([10], [12]) we proved that the map $t \mapsto \mu_{t}$ is differentiable for the weak $*$-topology. The series for $\Psi_{A}(1)$ may diverge (for the preperiodic case, see [7, §5]), but can be resumed under the horizontality condition [7], [10]. This gives an explicit linear response formula. In fact, the susceptibility function $\Psi_{A}(z)$ is holomorphic in the open unit disc, and, under a condition slightly stronger than horizontality, $\left.\partial_{t} \int A d \mu_{t}\right|_{t=0}$ is the Abel limit of $\Psi_{A}(z)$ as $z \rightarrow 1$.

Worrying about lack of differentiability of the SRB measure is not just a mathematician's pedantry: Indeed, this phenomenon can be observed numerically, for example in the guise of fractal transport coefficients. We refer, e.g., to the work of Keller et al. [28] (see also references therein), who obtained a $t \ln (t)$ modulus of continuity compatible with the results of [26], for drift and diffusion coefficients of models related to those analyzed in [10] [14].

Let us move on now to the topic of the present work, linear response for smooth unimodal interval maps: Ruelle recently obtained a linear response formula for real analytic families of analytic unimodal maps of Misiurewicz type [51], that is, assuming $\inf _{k}\left|f^{k}(c)-c\right|>0$, a nongeneric condition which implies the existence of a hyperbolic Cantor set. (Again, this linear response formula can be viewed as a resumation of the generally divergent series $\Psi_{A}(1)$.) In [11], we showed that $t \mapsto \mu_{t}$ is real analytic in the weak sense for complex analytic families of Collet-Eckmann quadratic-like maps (the - very rigid - holomorphicity assumption allowed us to use tools from complex analysis). Both these recent results are for families $f_{t}$ in the conjugacy class of a single (analytic) unimodal map, and the assumptions were somewhat nongeneric. 
The main result of the present work, Theorem 2.13, is a linear response formula for $C^{2}$ families $t \mapsto f_{t}$ of $C^{4}$ unimodal maps ${ }^{(1)}$ with quadratic critical points satisfying the so-called topological slow recurrence (TSR) condition ([54],[57],[32], see (5) below). (We assume that the maps have negative Schwarzian and are symmetric, to limit technicalities, and we only consider infinite postcritical orbits, since the preperiodic case is much easier.) The topological slow recurrence condition is much weaker than Misiurewicz, so that we give a new proof of Ruelle's result [51] in the symmetric infinite postcritical case (this may shed light on the informal study in $\S 17$ there). Topological slow recurrence implies the well-known BenedicksCarleson and Collet-Eckmann conditions. Furthermore, the work of Tsujii [57] and AvilaMoreira [6] gives that real-analytic unimodal maps with a quadratic critical point satisfying the TSR condition are measure-theoretical generic among non regular parameter in non trivial real-analytic families unimodal maps. (See Remark 2.3.) If all maps in a family of unimodal maps $f_{t}$ satisfy the topological slow recurrence condition then [55] this family is a deformation, that is, the family $\left\{f_{t}\right\}$ lies entirely in the topological class of $f_{0}$ (there exist homeomorphisms $h_{t}$ such that $h_{t}(c)=c$ and $h_{t} \circ f_{0}=f_{t} \circ h_{t}$ ). In particular, horizontality holds.

We next briefly discuss a few new ingredients of our arguments, as well as a couple of additional results we obtained along the way. A first remark is that we need uniformity of the hyperbolicity constants of $f_{t}$ for all small $t$. We deduce this uniformity from previous work of Nowicki, making use of the TSR assumption (Section 5).

When one moves the parameter $t$, the orbit of the critical point also moves, and so do the spikes. Therefore, in order to understand $\partial_{t} \mu_{t}$, we need upper bounds on

$$
\left.\partial_{t} c_{k, t}\right|_{t=0}=\left.\partial_{t} f_{t}^{k}(c)\right|_{t=0}=\left.\partial_{t} h_{t}\left(f_{0}^{k}(c)\right)\right|_{t=0}=\left.\partial_{t} h_{t}\left(c_{k, 0}\right)\right|_{t=0},
$$

uniformly in $k$. It is not very difficult to show (Lemma 2.10, see also Proposition 2.15) that $\left.\partial_{t} c_{k, t}\right|_{t=0}=\alpha\left(c_{k, 0}\right)$ if $\alpha$ solves the twisted cohomological equation (2) (TCE) for $v=\left.\partial_{t} f_{t}\right|_{t=0}$, given by,

$$
v=\alpha \circ f_{0}+f_{0}^{\prime} \cdot \alpha, \quad \alpha(c)=0 .
$$

(Such a function $\alpha$ is called an infinitesimal conjugacy.) In fact, we prove in Theorem 2.4 that if $f_{0}$ is Benedicks-Carleson and $v$ satisfies a horizontality condition for $f_{0}$, then the TCE above has a unique solution $\alpha$. In addition, $\alpha$ is continuous.

In the case of piecewise expanding maps on the interval, the invariant density $\phi_{t}$ is a fixed point of a Perron-Frobenius type transfer operator $\mathcal{L}_{t}$ in an appropriate space, where 1 is a simple isolated eigenvalue. So if we are able to verify some (weak) smoothness in the family $t \rightarrow \mathscr{L}_{t}$, then we can show (weak) differentiability of $\mu_{t}$ by using perturbation theory. (We may use different norms in the range and the domain, in the spirit of Lasota-Yorke or Doeblin-Fortet inequalities.) This is, roughly speaking, what was done in [10] and [13] (as already mentioned, a serious additional difficulty in the presence of critical points, which had to be overcome even in the toy model, is the absence of a spectral gap on a space containing the derivative of the invariant density). For Collet-Eckmann unimodal maps $f_{t}$, however, an

(1) The $C^{4}$ regularity is only used to get $W_{1}^{2}$ regularity in Proposition 4.11 and Lemma 4.12, and one can perhaps weaken this to $C^{3+\eta}$.

(2) In one-dimensional dynamics, the acronym TCE also stands [44] for "topological Collet-Eckmann," there should be no confusion since the topological Collet-Eckmann condition is not used here.

4 e SÉRIE - TOME $45-2012$ - No 6 
inducing procedure or a tower construction ([30], [61], [62]) is needed to obtain good spectral properties for the transfer operator and to properly analyse the density $\phi_{t}$, even for a single map.

We use the tower construction from [15], under a Benedicks-Carleson assumption. However, when we consider a one-parameter family of maps $f_{t}$, the phase space of the tower moves with $t$. To compare the operators for $f_{t}$ and $f_{0}$, it is convenient to work with a finite part of the tower, the height of which goes exponentially to infinity as $t \rightarrow 0$. (We use results of Keller and Liverani [29] to control the spectrum of the truncated operator.) The uniform boundedness of $\alpha\left(c_{k}\right)$ is instrumental in working with such truncated towers and operators. In fact, the tower construction in [15] also has a key role in the proof of boundedness for $\alpha$ : The natural candidate for the solution is a divergent series, but, under the horizontality condition, we devise a dynamical resumation (the mantra being: "don't perform a partial sum for the series while you are climbing the tower, unless you are ready to fall").

The tower from [15] has a drawback: The orbits of the edges of the tower levels apparently create "artificial discontinuities" in the functions. To eliminate these potential discontinuities, we modify the construction of the Banach spaces and transfer operators on the towers by introducing smooth cutoff functions (called $\xi_{k}$ below, see Section 4). As a consequence, we obtain a new expression for the invariant density of a Benedicks-Carleson unimodal map (Proposition 2.7), in terms of a single smooth function and of the dynamics.

We would like to list now a few directions for further work. Several of them can be explored by exploiting the techniques developed in the present article (see [8] for other open problems):

- In the setting of the present paper (for example), can one show that $\left.\partial_{t} \mu_{t}(A)\right|_{t=0}$ is a resumation of the divergent series $\Psi_{A}(z)$ at $z=1$ ? (Presumably, a dynamical resumation is possible, maybe using the operator $\mathscr{P}(\psi)=\psi \circ f$ dual to $\mathscr{L}$ acting on dual Banach spaces, and using, e.g., the proof of the main result in [23].) Can one get an Abelian limit along the real axis? The radius of convergence of $\Psi_{A}(z)$ is strictly smaller than 1 in general. There appears to be an essential boundary, except in the subhyperbolic cases when the critical point is preperiodic. Analytic continuation in the usual sense is thus probably not available, some kind of Borel or Abelian continuation seems necessary. (In subhyperbolic cases $\Psi_{A}(z)$ is meromorphic, and horizontality very likely implies vanishing of the residue of the pole in $[0,1]$.)

- Can one replace the topological slow recurrence condition on $f_{0}$ by Benedicks-Carleson, Collet-Eckmann, or possibly just a summability condition on the inverse of the postcritical derivative (see [43] and [17]), and still get differentiability ${ }^{(3)}$ of $t \mapsto \mu_{t}$, as a distribution of order 1 , at $t=0$ ?

- If $f_{t}$ is a smooth family of quadratic unimodal maps, with $f_{0}$ a good map (summable, or Collet-Eckmann, or Benedicks-Carleson, or TSR), and if $v=\left.\partial f_{t}\right|_{t=0}$ is horizontal for $f_{0}$,

(3) Perturbation theory of isolated eigenvalues cannot be used if there is no spectral gap, but the analysis in HairerMajda [23], e.g., indicates that existence of the resolvent (id $-\mathscr{L})^{-1}$ (up to replacing $\mathscr{L}$ by $\mathscr{P}$ if necessary) should be enough. 
that is, (10) holds ${ }^{(4)}$, is $t \mapsto \mu_{t}$ differentiable, as a distribution of order 1 , in the sense of Whitney, at $t=0$ ?

- If $f_{t}$ is a smooth (possibly transversal, that is, not horizontal) family of quadratic unimodal maps, with $f_{0}$ a good map, is $t \mapsto \mu_{t}$ always $r$-Hölder in the sense of Whitney for $r \in(0,1 / 2)$ at $t=0$ ? Which is the strongest topology one can use in the image? (Possibly, one could show Hölder continuity in the sense of Whitney of the Lyapunov exponent.)

- Can one construct a (non-horizontal) smooth family $f_{t}$ of quadratic unimodal maps, with $f_{0}$ a good map, so that $t \mapsto \mu_{t}$, as a distribution of any order, is not differentiable (even in the sense of Whitney, at least for large subsets) at $t=0$ ? So that it is not Hölder for any exponent $>1 / 2$ ?

- What about Hénon-like maps? Note that even the formula defining horizontality is not available in this case, see [8] (Numerical results of Cessac [20] indicate that $\Psi_{A}(z)$ has a singularity in the interior of the unit disc. In view of the above discussion, we expect that this singularity is not an isolated pole in general.)

- The dynamical zeta function associated to a Collet-Eckmann map $f$ and describing part of the spectrum of $\mathcal{L}$ was studied by Keller and Nowicki [30]. Can one study the analytic properties of a dynamical determinant for $\mathcal{L}$ in the spirit of what was done for subhyperbolic analytic maps [9]? (Analyticity would hold only in a disc of finite radius, and the correcting rational factor from [9, Theorem B] would be replaced by an infinite product, corresponding to the essential boundary of convergence within this disc.) Can one find and describe a dynamical determinant playing for $\Psi_{A}(z)$ the part that $\mathscr{L}$ plays for the Fourier transform of the correlation function of the SRB measure of $f$ ? (See [7] for piecewise expanding interval maps.)

The structure of the paper is as follows. In Section 2, we give precise definitions and state our main results formally. Section 3 is devoted to the proof (by dynamical resumation) that horizontality implies that the TCE has a continuous solution $\alpha$ (Theorem 2.4). In particular, we recall in Subsection 3.1 the construction of the tower map $\hat{f}: \hat{I} \rightarrow \hat{I}$ from [15] which will be used in later sections. We also show (Subsection 3.5) that the formal candidate for $\alpha$ diverges at countably many points (Proposition 2.5). In Section 4, we revisit the tower construction, introducing Banach spaces and a transfer operator $\widehat{\mathscr{L}}$ involving the smooth cutoff functions discussed above. In particular, Proposition 4.11, which immediately implies our new expression for the invariant density (Proposition 2.7), is proved in Subsection 4.1. Also, we study truncations $\widehat{\mathscr{L}}_{M}$ on finite parts of the tower in Subsection 4.2. Uniformity in $t$ of the hyperbolicity constants of $f_{t}$ involved in the construction of Sections 3 and 4 , is the topic of Section 5, the main result of which is Lemma 5.8 (proved by exploiting previous work of Nowicki). Finally, our linear response result, Theorem 2.13, is proved in Section 6. The argument borrows some ideas from [10], but their implementation required several nontrivial innovations, as explained above. The three appendices contain proofs of a more technical nature.

(4) By [4], we can heuristically view $f_{t}$ as tangent to the topological class of $f_{0}$.

4 e SÉRIE - TOME $45-2012$ - No 6 


\section{Formal statement of our results}

\subsection{Collet-Eckmann, Benedicks-Carleson, and topologically slowly recurrent (TSR) uni- modal maps}

We start by formally defining the classes of maps that we shall consider. Note that we shall sometimes write $[a, b]$ with $b<a$ to represent $[b, a]$. Another frequent abuse of notation is that we sometimes use $C>0$ to denote different (uniform) constants in the same formula.

Let $I=[-1,1]$. We say that $f$ is $S$-unimodal if $f: I \rightarrow I$ is a $C^{3}$ map with negative Schwarzian derivative such that $f(-1)=f(1)=-1,\left.f^{\prime}\right|_{[-1,0)}>0,\left.f^{\prime}\right|_{(0,1]}<0$, and $f^{\prime \prime}(0)<0$ (i.e., we only consider the quadratic case). The following notation will be convenient throughout: For $k \geq 1$, we let $J_{+}$be the monotonicity interval of $f^{k}$ containing $c$ and to the right of $c, J_{-}$be the monotonicity interval of $f^{k}$ containing $c$ and to the left of $c$, and we put

$$
f_{+}^{-k}:=\left(\left.f^{k}\right|_{J_{+}}\right)^{-1}, f_{-}^{-k}:=\left(\left.f^{k}\right|_{J_{-}}\right)^{-1}
$$

REMARK 2.1. - It is likely that the negative Schwarzian derivative assumption is not needed for our results, see [31]. Note however that we cannot apply trivially the work of Graczyk-Sands-Świątek [22] to study linear response: If $f_{t}$ is a one-parameter family of $C^{3}$ unimodal maps, the smooth changes of coordinates which make their Schwarzian derivative negative will depend on $t$, and this dependency will require a precise study. In view of keeping the length of this paper within reasonable bounds, we refrained from considering the more general case.

Let $c=0$ be the critical point of $f$, and put $c_{k}=f^{k}(c)$ for all $k \geq 0$. We say that an $S$-unimodal map $f$ is $\left(\lambda_{c}, H_{0}\right)$-Collet-Eckmann $(C E)$ if $\lambda_{c}>1, H_{0} \geq 1$, and

$$
\left|\left(f^{k}\right)^{\prime}(f(c))\right| \geq \lambda_{c}^{k}, \quad \forall k \geq H_{0} .
$$

All periodic orbits of Collet-Eckmann maps are repelling, and [42, Theorem B] gives that for any $C^{2}$ unimodal (or multimodal) map without periodic attractors there exists $\gamma>0$ so that $\left|f^{n}(c)-c\right| \geq e^{-\gamma n}$ for all large enough $n$. Benedicks and Carleson [16] showed that $S$-unimodal Collet-Eckmann maps which satisfy the following Benedicks-Carleson assumption

$$
\exists 0<\gamma<\frac{\ln \left(\lambda_{c}\right)}{\mathscr{G}} \text { so that }\left|f^{k}(c)-c\right| \geq e^{-\gamma k}, \quad \forall k \geq H_{0}
$$

for $\mathscr{G}=4$, form a positive measure set of parameters of non degenerate families. The Benedicks-Carleson assumption will suffice for some of our results, sometimes replacing $\mathscr{G}=4$ by a larger constant.

A stronger condition, topologically slow recurrence (TSR), will allow us to obtain linear response. To define TSR, we shall use the following auxiliary sequence: Let $f$ be an $S$-unimodal map whose critical point is not preperiodic. The itinerary of a point $x \in I$ is the sequence $\operatorname{sgn}\left(f^{i}(x)\right) \in\{-1,0,1\}$. We put

$$
R_{f}(x):=\min \left\{j \mid \operatorname{sgn}\left(f^{j}(c)\right) \neq \operatorname{sgn}\left(f^{j}(x)\right), j \geq 1\right\} .
$$


We say that an $S$-unimodal map $f$ with non preperiodic critical point satisfies the topological slow recurrence (TSR) condition if

$$
\lim _{m \rightarrow \infty} \limsup _{n \rightarrow \infty} \frac{1}{n} \sum_{\substack{1 \leq j \leq n \\ R_{f}\left(f^{j}(c)\right) \geq m}} R_{f}\left(f^{j}(c)\right)=0 .
$$

It follows from the definition that any $S$-unimodal map topologically conjugated with a map $f$ satisfying TSR also satisfies TSR. (Indeed, $R_{f}\left(c_{n}\right)=j$ if and only if $f^{j}$ is a diffeomorphism on $\left(c, c_{n}\right)$ and $c \in f^{j}\left(c, c_{n}\right)$.) We also have the much less trivial result below:

Proposition 2.2 ([54], [60]. See also [32]). - An S-unimodal map $f$ with non preperiodic critical point satisfies the TSR condition if and only if $f$ is a Collet-Eckmann map and

$$
\lim _{\eta \rightarrow 0^{+}} \liminf _{n \rightarrow \infty} \frac{1}{n} \sum_{\substack{1 \leq j \leq n \\\left|f^{j}(c)-c\right|<\eta}} \ln \left|f^{\prime}\left(f^{j}(c)\right)\right|=0 .
$$

In Section 5, we shall prove that TSR implies Collet-Eckmann and Benedicks-Carlesontype conditions, uniformly in a subset of small enough $C^{3}$ diameter of a topological class.

REMARK 2.3 (TSR is generic). - Avila and Moreira [5] proved that for almost every parameter $s$ in a non-degenerate analytic family of quadratic unimodal maps $f_{s}$, the map $f_{s}$ is either regular or Collet-Eckmann with subexponential recurrence of its critical orbit (i.e., for every $\gamma>0$, there is $H_{0}$ so that $\left|c_{k}-c\right|>\exp (-\gamma k)$ for all $\left.k \geq H_{0}\right)$. (Non-degenerate, or transversal, means that the family is not contained in a topological class.) Tsujii [57] had previously proved that the set of Collet-Eckmann and subexponentially recurrent parameters $s$ in a transversal family $f_{s}$ of $S$-unimodal maps has positive Lebesgue measure. By combining the results of Avila and Moreira [5] and Tsujii [57], we can see that TSR is a generic condition: In a nondegenerate analytic family $f_{s}$ of $S$-unimodal maps, almost every parameter is either regular or TSR.

\subsection{Boundedness and continuity of the infinitesimal conjugacy $\alpha$}

Let $f$ be an $S$-unimodal Collet-Eckmann map, and let $v: I \rightarrow \mathbb{C}$ be bounded. We want to find a bounded solution $\alpha: I \rightarrow \mathbb{C}$ of the twisted cohomological equation (TCE):

$$
v(x)=\alpha(f(x))-f^{\prime}(x) \alpha(x), \forall x \in I .
$$

By analogy with the piecewise expanding unimodal case (that we studied in previous works [10], [12]), a candidate $\alpha_{\text {cand }}$ for the solution $\alpha$ of (7) is defined, for those $x \in I$ so that $f^{j}(x) \neq c$ for all $j \geq 0$, by the formal series

$$
\alpha_{\text {cand }}(x)=-\sum_{j=0}^{\infty} \frac{v\left(f^{j}(x)\right)}{\left(f^{j+1}\right)^{\prime}(x)},
$$

and, for those $x \in I$ so that there exists $\ell \geq 0$ with $f^{\ell}(x)=c$, but $f^{j}(x) \neq c$ for $0 \leq j \leq \ell-1$, by the sum

$$
\alpha_{\text {cand }}(x)=-\sum_{j=0}^{\ell-1} \frac{v\left(f^{j}(x)\right)}{\left(f^{j+1}\right)^{\prime}(x)} .
$$

$4^{\text {e }}$ SÉRIE - TOME $45-2012-\mathrm{N}^{\circ} 6$ 
(In particular, $\alpha_{\text {cand }}(c)=0$.) Clearly, the series (8) converges absolutely at every point $x$ for which the Lyapunov exponent

$$
\Lambda(x)=\lim _{j \rightarrow \infty} \ln \left|\left(f^{j}\right)^{\prime}(x)\right|^{1 / j}
$$

is well-defined and strictly positive. In particular, (8) converges absolutely for $x$ in the forward orbit $\left\{c_{k}, k \geq 1\right\}$ of the critical point of the Collet-Eckmann $S$-unimodal map $f$, and also on the set of its preperiodic points.

We say that $v$ satisfies the horizontality condition if

$$
v(c)=-\sum_{j=0}^{\infty} \frac{v\left(f^{j}\left(c_{1}\right)\right)}{\left(f^{j+1}\right)^{\prime}\left(c_{1}\right)},
$$

(note that the right-hand side of the above identity is just $\alpha_{\text {cand }}\left(c_{1}\right)$ ). If $v$ satisfies the horizontality, then it is easy to see that whenever the formal series (8) for $\alpha_{\text {cand }}(x)$ converges absolutely, then the corresponding series $\alpha_{\text {cand }}(f(x))$ also converges absolutely, and $\alpha_{\text {cand }}$ satisfies the twisted cohomological Equation (7) at $x$. Violation of horizontality (that is, $\left.v(c) \neq \alpha_{\text {cand }}\left(c_{1}\right)\right)$ is a transversality condition which has been used for a long time in oneparameter families $f_{t}$ of smooth unimodal maps with $v(x)=\left.\partial_{t} f_{t}\right|_{t=0}$ (see, e.g., [56] for the transversality condition, see [57] for the transversality condition expressed as a postcritical sum, see, e.g., [12, §5] for the link between the two expressions, see [4] for a recent occurrence, and see [51] for its use in linear response).

Nowicki and van Strien [43] showed that the absolutely continuous invariant probability measure $\mu$ of a quadratic Collet-Eckmann map satisfies

$$
\mu(A) \leq C m(A)^{1 / 2},
$$

where $m$ is the Lebesgue measure. In particular $\ln \left|f^{\prime}\right|$ is $\mu$-integrable, and for Lebesgue almost every point $x$ the Lyapunov exponent $\Lambda(x)$ is well-defined and positive and coincides with $\int \ln \left|f^{\prime}\right| d \mu$ (see Keller [27]). So the series $\alpha_{\text {cand }}(x)$ converges absolutely at Lebesgue almost every point $x$, and if $v$ is horizontal then $\alpha_{\text {cand }}$ satisfies the TCE (7) along the forward orbit of each such good $x$. However it is not clear a priori that there exists an upper bound for $\left|\alpha_{\text {cand }}(x)\right|$ on the set where $\alpha_{\text {cand }}(x)$ converges absolutely (for example, $c_{k}$ may be very close to $c$ ).

One can ask whether the formal series $\alpha_{\text {cand }}(x)$ converges everywhere. We shall show in Proposition 2.5 that for fairly general $v$ (see Remark 2.6), the series (8) for $\alpha_{\text {cand }}(x)$ diverges on an uncountable and dense subset (this set has Lebesgue measure zero, however, by the observations in the previous paragraph). This lack of convergence is a new phenomenon with respect to [10], [12]. In order to prove that the TCE nevertheless has a bounded solution in the horizontal case, we shall make a Benedicks-Carleson assumption (3) (with $\mathscr{G}=4$ ) on $f$, and we shall group the terms of the formal series $\alpha_{\text {cand }}(x)$ to obtain an absolutely convergent series. The resumation procedure depends on $x$ through its dynamics with respect to an induced map on the tower introduced in [15], using strong expansion properties available in the Benedicks-Carleson case. This dynamical resumation will allow us to prove our first main result:

THeorem 2.4 (Boundedness and continuity of $\alpha$ ). - Assume that $f$ is a $\left(\lambda_{c}, H_{0}\right)$-ColletEckmann $S$-unimodal map satisfying the Benedicks-Carleson condition (3) with $\mathscr{G}=4$. 
For any bounded function $v: I \rightarrow \mathbb{C}$, if the TCE (7) admits a bounded solution $\alpha: I \rightarrow \mathbb{C}$ with $\alpha(c)=0$, then this solution is unique and $v$ satisfies the horizontality condition (10).

Let $X: I \rightarrow \mathbb{C}$ be Lipschitz, and let $v=X \circ f$. If $v$ satisfies the horizontality condition (10), then there exists a continuous function $\alpha: I \rightarrow \mathbb{C}$ with $\alpha(c)=0$ solving the TCE (7). In addition, $\alpha(x)=\alpha_{\text {cand }}(x)$ for all $x$ so that $f^{j}(x)=c$ for some $j \geq 0$, or so that the infinite series $\alpha_{\text {cand }}(x)$ in (8) converges absolutely.

The condition $v=X \circ f$ can be weakened but the term $k=0$ of $(I)$ in (47) in the proof of Proposition 3.9 shows that we need something like $v^{\prime}(c)=0$. (If we allowed $f^{\prime \prime}(c)=0$, then we would need $v^{\prime \prime}(c)=0$, etc.)

We do not know whether Theorem 2.4 holds for all $S$-unimodal Collet-Eckmann maps, i.e., whether the Benedicks-Carleson assumption is needed. In any case, we shall use the stronger, but still generic (recall Remark 2.3), TSR assumption in Section 5 to show uniformity of the various hyperbolicity constants. This uniformity is required to prove linear response, the other main result of this paper.

The proof of Theorem 2.4 is given in Section 3 and organized as follows: In Section 3.1, we recall the tower construction from Baladi and Viana [15]. We study its properties in Section 3.2, which also contains two new (and key) estimates, Proposition 3.7 and its Corollary 3.8. In Section 3.3, we define a function $\alpha(x)$ by grouping the terms of the formal series (8) to obtain an absolutely convergent series (Definition 3.10 and Proposition 3.9). The resumation procedure for $\alpha(x)$ depends on the dynamics of $x$ on the tower. Finally, in Section 3.4 we complete the proof of Theorem 2.4: We show that $\alpha(x)$ is a continuous function, that it satisfies the TCE, and that if the TCE admits a bounded solution then it is unique.

We end this section with a result on the lack of convergence of the formal power series for $\alpha_{\text {cand }}(x)$ (recall that it converges at Lebesgue almost every $x$ ):

Proposition 2.5. - Let $f$ be an $S$-unimodal map, with all its periodic points repelling and an infinite postcritical orbit. Let $v$ be a $C^{1}$ function on $I$, with $v^{\prime}(c)=0$, such that $v\left(f^{i_{0}}(c)\right) \neq 0$ for some $i_{0}$. Let $\Sigma$ be the set of points $x$ such that $f^{n}(x) \neq c$ for every $n \geq 0$ and so that the series $\alpha_{\text {cand }}(x)=-\sum_{i=0}^{\infty} \frac{v\left(f^{i}(x)\right)}{\left(f^{i+1}\right)^{\prime}(x)}$ diverges. Then for every non empty open set $A \subset I$, the intersection $A \cap \Sigma$ contains a Cantor set.

REMARK 2.6. - If $f$ is a Collet-Eckmann map whose critical orbit is not preperiodic, an open and dense set of horizontal vectors $v$ satisfies the conditions of Proposition 2.5. Indeed, the set $\left\{v \mid v\left(f^{i}(c)\right)=0, \forall i\right\}$ is a subspace of infinite codimension, and the subspace of horizontal directions $v$ has codimension one.

The proof of Proposition 2.5 is to be found in Section 3.5.

4 e SÉRIE - TOME $45-2012$ - No 6 


\subsection{A new expression for the a.c.i.m. of a Benedicks-Carleson unimodal map}

It is well-known that an $S$-unimodal map which is Collet-Eckmann admits an absolutely continuous invariant measure. The following expression for the invariant density of a Benedicks-Carleson unimodal map appears to be new. It is a byproduct of our proof, and follows immediately from Proposition 4.11 and the definitions in Section 4 (the case when the critical point is preperiodic can be obtained by a much more elementary proof). The remarkable feature of (11) is that the defining function $\psi_{0}$ is smooth, and that the square-root singularities appear dynamically, through the inverse iterates of $f$ and their jacobians.

Proposition 2.7. - Let $f$ be a $\left(\lambda_{c}, H_{0}\right)$-Collet-Eckmann $S$-unimodal map satisfying the Benedicks-Carleson condition (3) for $\mathscr{G}=14$, with c not preperiodic. If $f$ is $C^{4}$, then there exist

- a $C^{1}$ function $\psi_{0}: I \rightarrow \mathbb{R}_{+}$, which belongs to the Sobolev space $W_{1}^{2}$,

- for each $k \geq 1$, neighborhoods $V_{k} \subset W_{k}$ of $c=0$, so that $\left.f^{k}\right|_{W_{k} \cap[0,1]}$ and $\left.f^{k}\right|_{[-1,0] \cap W_{k}}$ are injective,

- for each $k \geq 1$, a $C^{\infty}$ function $\xi_{k}: I \rightarrow[0,1]$, supported in $W_{k}$ and $\equiv 1$ on $V_{k}$,

so that the density $\phi$ of the unique absolutely continuous invariant probability measure of $f$ satisfies

$$
\phi(x)=\psi_{0}(x)+\sum_{k=1}^{\infty} \sum_{\varsigma \in\{+,-\}} \frac{\prod_{j=1}^{k-1} \xi_{j}\left(f_{\varsigma}^{-k}(x)\right)}{\left|\left(f^{k}\right)^{\prime}\left(f_{\varsigma}^{-k}(x)\right)\right|} \chi_{k}(x) \psi_{0}\left(f_{\varsigma}^{-k}(x)\right),
$$

where $\chi_{k}=1_{\left[-1, c_{k}\right]}$ if $f^{k}$ has a local maximum at $c$, while $\chi_{k}=1_{\left[c_{k}, 1\right]}$ if $f^{k}$ has a local minimum at $c$.

There is some flexibility in choosing the (exponentially decaying) intervals $V_{k}, W_{k}$, and the functions $\xi_{k}$, see Definition 4.7 for details, noting also the parameter $\delta$ used in the construction of the tower. The function $\psi_{0}$ depends on these choices.

By Lemma 4.1, which describes the nature of the singularities of $\left|\left(f^{k}\right)^{\prime} f_{ \pm}^{-k}(x)\right|$ on the support of $\xi_{k}\left(f_{ \pm}^{-k}(x)\right)$, the expression for $\phi$ belongs to $L^{p}(I)$ for all $p<2$ (this fact is well known, and was proved recently [18] under much weaker assumptions). In fact, Lemma 4.1 (see also (75)) implies that the invariant density of $f$ can be written as

$$
\psi_{0}+\sum_{k \geq 1} \phi_{k} \frac{\chi_{k}}{\sqrt{\left|x-c_{k}\right|}}
$$

where the $C^{1}$ norms of the $\phi_{k}$ decay exponentially with $k$. (A slightly weaker version of this result, replacing differentiable by bounded variation, was first proved by L.S. Young [61]. Ruelle obtained a formula involving differentiable objects in the analytic Misiurewicz case [51], but his expression is somewhat less dynamical.)

\subsection{Uniformity of hyperbolicity constants in deformations of slowly recurrent maps}

We shall study one-parameter families $t \mapsto f_{t}$ of $S$-unimodal maps which stay in a topological class, i.e., deformations: 
Definition $2.8\left(C^{r}\right.$ deformations $f_{t}$. Notations $\left.v_{t}, X_{t}, h_{t}\right)$. - Let $f: I \rightarrow I$ be a $S$-unimodal Collet-Eckmann map. For $r \geq 1$, a $C^{r}$ one-parameter family through $f$ is a $C^{r}$ map

$$
t \mapsto f_{t}, t \in[-\epsilon, \epsilon],
$$

(taking the topology of $C^{3}$ endomorphisms of $I$ in the image), with $f_{0}=f$, and so that each $f_{t}$ is $S$-unimodal. We use the notations:

$$
c_{k, t}=f_{t}^{k}(c), k \geq 1, v_{s}:=\left.\partial_{t} f_{t}\right|_{t=s}, v=v_{0}, c_{k}=c_{k, 0} .
$$

A $C^{r}$ deformation of $f$ is a $C^{r}$ one-parameter family through $f$ so that, in addition, for each $|t| \leq \epsilon$, there exists a homeomorphism $h_{t}: I \rightarrow I$ with

$$
h_{0}(x) \equiv x, \text { and } f_{t} \circ h_{t}=h_{t} \circ f_{0}, \forall t \in[-\epsilon, \epsilon],
$$

and ${ }^{(5)} v_{s}=X_{s} \circ f_{s}$ for each $|s| \leq \epsilon$, with $X_{s}: I \rightarrow \mathbb{R}$ a $C^{2}$ function. (We write $X=X_{0}$.)

REMARK 2.9. - If $f_{t}$ is a deformation then each $v_{s}$ is horizontal. (This was proved by Tsujii [57].)

Given Theorem 2.4, the next lemma is easy to prove. It is essential in our argument:

Lemma 2.10. - Let $f_{t}$ be a $C^{1}$ one-parameter family of Collet-Eckmann $S$-unimodal maps through $f=f_{0}$. Assume that $v$ is horizontal, that is, $\alpha_{\text {cand }}\left(c_{1}\right)=v(c)$ (by Theorem 2.4, the TCE then admits a unique bounded solution $\alpha$ ).

Then, we have for all $k \geq 1$

$$
\lim _{t \rightarrow 0} \frac{c_{k, t}-c_{k}}{t}=\alpha_{\text {cand }}\left(c_{k}\right) .
$$

If, in addition, $f_{t}$ is a $C^{1}$ deformation of $f_{0}$ then

$$
\left.\partial_{t} h_{t}\left(c_{k}\right)\right|_{t=0}=\alpha_{\text {cand }}\left(c_{k}\right)=\alpha\left(c_{k}\right), \quad \forall k \geq 1 .
$$

Proof. - Our assumptions ensure that for each $k \geq 1$ the limit

$$
a\left(c_{k}\right)=\lim _{t \rightarrow 0} \frac{c_{k, t}-c_{k}}{t}
$$

exists. Clearly, $a\left(c_{1}\right)=v(c)$. More generally, it is easy to check that we have

$$
a\left(c_{k}\right)=\sum_{j=0}^{k-1}\left(f^{j}\right)^{\prime}\left(c_{k-j}\right) v\left(c_{k-j-1}\right),
$$

so that $a$ satisfies the TCE (7). By the horizontality condition, this implies that $a=\alpha_{\text {cand }}$ on $\left\{c_{k}\right\}_{k \geq 0}$.

The additional assumption that $f_{t}$ and $f_{0}$ are conjugated via $h_{t}$ implies that $h_{t}\left(c_{k}\right)=c_{k, t}$, for all $t$ and all $k \geq 0$. The last statement of Theorem 2.4 implies that $\alpha_{\text {cand }}\left(c_{k}\right)=\alpha\left(c_{k}\right)$ for all $k \geq 1$.

The following fact is an immediate consequence of van Strien's remark on "robust chaos" [55, Theorem 1.1], using the well-known fact that Collet-Eckmann maps do not have any attracting periodic orbit:

(5) This is mostly a technical assumption, see also the remark after Theorem 2.4.

4 e SÉRIE - TOME $45-2012$ - No 6 
Lemma 2.11. - Let $f: I \rightarrow I$ be an $S$-unimodal $\left(\lambda_{c}, H_{0}\right)$ Collet-Eckmann map and let $f_{t}$ be a $C^{1}$ one-parameter family through $f$. If each $f_{t}$ is Collet-Eckmann for some parameters $\lambda_{c}(t)$ and $H_{0}(t)$, then $f_{t}$ is a $C^{1}$ deformation of $f$.

In the other direction, although topological invariance of the Collet-Eckmann condition is known, we do not know how to prove topological invariance of Benedicks-Carleson conditions (3). Since we also need uniformity of the various constants in the definitions, we shall work with the stronger, but still generic (see Remark 2.3), assumption of topological slow recurrence TSR (recall (5)). In Section 5, assuming for simplicity that the maps are symmetric, we shall prove that if $f_{s}$ is a $C^{0}$ deformation of a TSR map $f_{0}$ then the various hyperbolicity constants of $f_{s}$ (that is $\lambda_{c}\left(f_{s}\right)$ and $H_{0}\left(f_{s}\right)$, but also $\sigma\left(f_{s}\right), c_{f_{s}}(\delta)^{-1}, \rho\left(f_{s}\right)$ from Subsection 3.2, and, especially, $\left.\gamma\left(f_{s}\right)\right)$ are uniform in small $s$. We refer to Lemma 5.8 for a precise statement. Also, it will follow from Propositions 5.1 and 5.2 that (TSR) implies (CE) and the Benedicks-Carleson condition (3).

The uniformity of the constants from Lemma 5.8 implies in particular that, if $f_{t}$ is a $C^{1}$ deformation of symmetric $S$-unimodal maps so that $f_{0}$ enjoys topological slow recurrence TSR, then the assumptions of Theorem 2.4 are satisfied for each $f_{s}$. We may thus state a lemma which will be essential in many places in the proof of Theorem 2.13:

Lemma 2.12. - Let $f_{t}$ be a $C^{1}$ deformation of symmetric $S$-unimodal maps so that $f_{0}$ enjoys topological slow recurrence TSR. Denote by $\alpha_{t}$ the continuous solution to the TCE given by Theorem 2.4 applied to $f_{t}$. Then there exist $\epsilon>0$ and $L<\infty$ so that

$$
\sup _{x} \sup _{|t|<\epsilon}\left|\alpha_{t}(x)\right| \leq L
$$

and

$$
\left|c_{k}-c_{k, t}\right| \leq L|t| \quad \forall k \geq 1, \quad \forall|t|<\epsilon
$$

The proof of Lemma 2.12 is simple, but since it uses notation to be introduced later on, we postpone it until after the proof of Lemma 5.8.

\subsection{Linear response}

Our main result will be proved in Section 6:

THEOREM 2.13 (Linear response and linear response formula). - Let $\eta>0$ and let $f_{t}$ be $a C^{2}$ deformation of a $C^{4} S$-unimodal map $f_{0}$ which satisfies TSR. Assume that all maps $f_{t}$ are symmetric. Write $\mu_{t}=\phi_{t} d x$ for the unique absolutely continuous invariant probability of $f_{t}$. Then, letting $\left(C^{1}(I)\right)^{*}$ be the dual of $C^{1}(I)$, the map

$$
t \mapsto \mu_{t} \in\left(C^{1}(I)\right)^{*}, t \in[-\epsilon, \epsilon]
$$

is differentiable. In addition, for any $A \in C^{1}(I)$,

$$
\left.\int_{I}\left(A-A \circ f_{s}\right) \partial_{t} \mu_{t}\right|_{t=s}=\int_{I} A^{\prime} X_{s} \phi_{s} d x
$$


Formula (16) is an easy consequence of the differentiability of (15), as was pointed out to us by Ruelle [52].

We next give an explicit formula for $\left.\partial_{t} \mu_{t}\right|_{t=0}$ (we choose $t=0$ for definiteness). For this, we need further notation. Introduce $Y_{k, s}=\lim _{t \rightarrow s} \frac{f_{t}^{k}-f_{s}^{k}}{t-s}$ for $k \geq 1$ (we write $Y_{k}$ instead of $\left.Y_{k, 0}\right)$. Then $Y_{1, s}=X_{s} \circ f_{s}, Y_{2, s}=X_{s} \circ f_{s}^{2}+\left(f_{s}^{\prime} \circ f_{s}\right) X_{s} \circ f_{s}$, and

$$
Y_{k, s}=\sum_{j=1}^{k}\left(\left(f_{s}^{k-j}\right)^{\prime} \circ f_{s}^{j}\right) \cdot X_{s} \circ f_{s}^{j}, k \geq 1 .
$$

Put (note the shift in indices!)

$$
\hat{Y}_{s}=\left(\hat{Y}_{s}(x, k)=Y_{k+1, s}(x), k \geq 0\right), \quad \hat{Y}=\hat{Y}_{0} .
$$

Referring to Section 4 for the definitions of $\lambda, \widehat{\mathscr{L}}, \hat{\phi}$, $\Pi$, and $\mathcal{T}_{0}$, and summing (131) and (150) from the proof of Theorem 2.13, we get

$$
\begin{aligned}
\int A \partial_{t} & \left.\mu_{t}\right|_{t=0} \\
& =-\int A \cdot \Pi\left((\mathrm{id}-\widehat{\mathscr{L}})^{-1} \mathcal{J}_{0}(\widehat{\mathscr{L}}(\hat{Y} \hat{\phi}))^{\prime} d x-\lambda \int A^{\prime} \cdot \Pi\left(\left(\mathrm{id}-\mathcal{T}_{0}\right)(\widehat{\mathscr{L}}(\hat{Y} \hat{\phi}))\right) d x\right.
\end{aligned}
$$

Using the definitions of $\widehat{\mathcal{L}}, \hat{\phi}, \Pi$, and $\mathcal{T}_{0}$, the linear response formula above can be rewritten in terms of $f$ and the functions $\psi_{0}, \xi_{k}$ and $\chi_{k}$ from Proposition 2.7. The reader can then compare this rewriting to the expression in [51, 17 and §18], obtained under the additional assumptions that $f_{0}$ is Misiurewicz and all the $f_{t}$ are real analytic. (See also Remark 2.14 below.)

We next discuss (16). Using (133), we find

$$
\left.\int(A-A \circ f) \partial_{t} \mu_{t}\right|_{t=0}=\int A^{\prime} \mathcal{T}_{0}(\widehat{\mathscr{L}}(\hat{Y} \hat{\phi})) d x-\lambda \int\left(A^{\prime}-(A \circ f)^{\prime}\right) \cdot \Pi\left(\left(\mathrm{id}-\mathcal{T}_{0}\right) \widehat{\mathscr{L}}(\hat{Y} \hat{\phi})\right) d y \text {. }
$$

By Theorem 2.13, the right-hand side above coincides with the expression (16). We sketch here a direct proof of this fact: The left-hand side above being independent of the parameter $\delta$ used in the construction of the tower (note however that $\Pi, \widehat{\mathcal{L}}$, and $\hat{\phi}$ depend on $\delta$ ), we can let $\delta \rightarrow 0$.

We expect that, when $\delta \rightarrow 0$, the function $\phi_{0}$ converges to $\phi$ in the $L^{1}$ topology, and that for any continuous function $B$, on the one hand, we have

$$
\lim _{\delta \rightarrow 0} \int_{I} B \cdot \Pi\left(\left(\mathrm{id}-\mathcal{T}_{0}\right)(\widehat{\mathcal{L}}(\hat{Y} \hat{\phi}))\right) d y=0
$$

and on the other hand, using in particular (134) and the facts that ${ }^{(6)} Y_{1}=X_{0} \circ f_{0}$ and $\widehat{\mathscr{L}}(\hat{\phi})=\hat{\phi}$, we get

$$
\lim _{\delta \rightarrow 0} \int B \mathcal{T}_{0}(\widehat{\mathscr{L}}(\hat{Y} \hat{\phi})) d x=\int B X \phi d x
$$

(6) Note also that, as $\delta \rightarrow 0$, the smallest level from which points may fall from the tower tends to infinity. 
ReMARK 2.14. - If $\mathscr{L}$ denotes the transfer operator defined on distributions $v$ of order one by $\int A \mathscr{L} v=\int(A \circ f) v$, for all $C^{1}$ functions $A$, expression (16) can be written as a left inverse (both sides should be viewed as distributions of order one)

$$
\left.\partial_{t} \mu_{t}\right|_{t=0}=-(\mathrm{id}-\mathscr{L})_{L}^{-1}(X \phi)^{\prime} d x
$$

Indeed

$$
\left.\int(A-A \circ f) \partial_{t} \mu_{t}\right|_{t=0}=\left.\int A(\mathrm{id}-\mathscr{L}) \partial_{t} \mu_{t}\right|_{t=0},
$$

and, in the sense of distributions (writing $\mu=\mu_{0}$ as usual),

$$
\int A^{\prime} X \phi d x=-\int A(X \phi)^{\prime} d x=-\int A \operatorname{div}_{\mu}(X) d \mu .
$$

We next explain the heuristics of the connection with the susceptibility function. In situations where more information is available (such as smooth expanding circle maps), the following formal manipulations become licit (they are not licit in the present case of smooth unimodal maps, in particular the sum below diverges in general):

$$
\begin{aligned}
\int A(\mathrm{id}-\mathcal{L})_{L}^{-1}(X \phi)^{\prime} d x & =\int A \sum_{j=0}^{\infty} \mathcal{L}^{j}(X \phi)^{\prime} d x \\
& =\int \sum_{j=0}^{\infty} \mathcal{L}^{j}\left(\left(A \circ f^{j}\right)(X \phi)^{\prime}\right) d x=-\sum_{j=0}^{\infty} \int\left(A \circ f^{j}\right)^{\prime} X \phi d x
\end{aligned}
$$

so that

$$
\left.\int A \partial_{t} d \mu_{t}\right|_{t=0}=\sum_{j=0}^{\infty} \int\left(A \circ f^{j}\right)^{\prime} X d \mu=\Psi_{A}(1) .
$$

We end this section with a result that we shall not need, but which is of independent interest (the proof is given in Appendix A):

Proposition 2.15 (The solution of the TCE is an infinitesimal conjugacy)

Let $t \mapsto f_{t}$ be a $C^{1}$ deformation of the $S$-unimodal $\left(\lambda_{c}, H_{0}\right)$ Collet-Eckmann map $f_{0}$. Assume furthermore that for each $|t| \leq \epsilon$ there exists a unique continuous function $\alpha_{t}$ on $I$ which solves the TCE (7) for $v=v_{t}:=\left.\partial_{s} f_{s}\right|_{s=t}$ and $f=f_{t}$, and in addition that the family $\left\{\alpha_{t}\right\}_{|t| \leq \epsilon}$ of ${ }^{(7)}$ continuous maps is equicontinuous. Then for each $x \in I$ the function $t \mapsto h_{t}(x)$ is $C^{1}$, and

$$
\left.\partial_{s} h_{s}(x)\right|_{s=t}=\alpha_{t}\left(h_{t}(x)\right), \quad \forall t \in(-\epsilon, \epsilon) .
$$

Note that if each $f_{t}$ satisfies the Benedicks-Carleson assumption (3) for $\mathscr{G}=4$ then Theorem 2.4 ensures that the TCE associated to $f_{t}$ and $v_{t}$ has a unique solution $\alpha_{t}$, which is continuous (recall Remark 2.9). We expect that equicontinuity of the family $\alpha_{t}$ can be obtained, possibly under the topological slow recurrence condition TSR.

(7) By compactness of $I$ and $[-\epsilon, \epsilon]$, continuity is equivalent to uniform continuity here. 


\section{Proof of Theorem 2.4: Boundedness and continuity of the solution $\alpha$ of the TCE}

\subsection{The tower map $\hat{f}$, the times $S_{i}(x)$ and $T_{i}(x)$, and the intervals $I_{j}$}

Before recalling the tower construction from [15], we mention crucial expansion properties of Collet-Eckmann maps which improve ${ }^{(8)}$ over [15, Lemma 1]:

Lemma 3.1 (Collet-Eckmann maps expansion). - Let $f$ be an $S$-unimodal $\left(\lambda_{c}, H_{0}\right)$-Collet-Eckmann map.

There exist $\sigma=\sigma_{f}>1$ and $C=C_{f}>0$ and for every small $\delta>0$ there exists $c(\delta)=c_{f}(\delta)$ such that for any $j \geq 1$

$$
\left|\left(f^{i}\right)^{\prime}(x)\right| \geq c(\delta) \sigma^{i}, \forall 0 \leq i \leq j, \forall x \text { so that }\left|f^{k}(x)\right|>\delta, \forall 0 \leq k<j .
$$

For every $1<\rho<\sqrt{\lambda_{c}}$ there exists $C_{1}=C_{1}(\rho) \in(0,1]$ and for each $\delta_{0}>0$ there exists $\delta \in\left(0, \delta_{0}\right)$ such that for any $j \geq 1$

$$
\left|\left(f^{j}\right)^{\prime}(x)\right| \geq C_{1} \rho^{j}, \forall x \text { so that }\left|f^{i}(x)\right|>\delta, \forall 0 \leq i<j,\left|f^{j}(x)\right| \leq \delta .
$$

In addition, we can assume that either $\pm \delta$ are preperiodic points, or that they have infinite orbits and that their Lyapunov exponents exist and are strictly positive.

REMARK 3.2. - Except in Remarks (19) and (20), and, more importantly, in Remark 4.9 (which is used in Appendix B), we do not use that $c(\delta) \geq C \delta$, only that $c(\delta)>0$ if $\delta>0$.

Proof. - By Theorem 7.7 in [41], there exist $\sigma>1$ and $K>0$ such that

$$
\left|\left(f^{i}\right)^{\prime}(x)\right| \geq K \sigma^{i} \min _{0 \leq k<i}\left|f^{\prime}\left(f^{k}(x)\right)\right| .
$$

Since $\left|f^{\prime}(y)\right| \geq \widetilde{K}|y|$ and $\left|f^{k}(x)\right| \geq \delta$ for $k<j$ and $i \leq j$, we have (22).

To prove (23), we use Proposition 3.2(6) in [39] which says that for every $1<\rho<\lambda_{c}^{1 / 2}$ there exists $\widetilde{C}$ such that if $f^{j}(y)=c$ then

$$
\left|\left(f^{j}\right)^{\prime}(y)\right| \geq \widetilde{C} \rho^{j} .
$$

By Theorem 3.2 in [33] and the Koebe lemma, there exist $K>0$ and arbitrarily small $\delta>0$ such that the following holds: if $\left|f^{i}(x)\right|>\delta$ for $0 \leq i<j$ and $\left|f^{j}(x)\right| \leq \delta$ then there exists an interval $J$, with $x \in J$, such that $f^{j}(J)=[-\delta, \delta], f^{j}$ is a diffeomorphism on $J$, and

$$
\frac{1}{K} \leq \frac{\left|\left(f^{j}\right)^{\prime}(y)\right|}{\left|\left(f^{j}\right)^{\prime}(x)\right|} \leq K, \forall y \in J
$$

Let $y \in J$ be such that $f^{j}(y)=c$. By (24) and (25) it follows that

$$
\left|\left(f^{j}\right)^{\prime}(x)\right| \geq \frac{\widetilde{C}}{K} \rho^{j}
$$

Finally we can choose $\delta$ so that $\pm \delta$ are preperiodic points, or $\pm \delta$ non preperiodic with $\Lambda( \pm \delta)$ well-defined and positive. Indeed by [34], $f$ admits an expanding induced map $M$ that is a $C^{3}$ Markov map. In particular $M: \cup_{i} J_{i} \rightarrow J$, where $J_{i}$ and $J$ are open intervals, $c \in J$, $\cup_{i} J_{i}$ is dense in $J, M(x)=f^{n_{i}}(x)$ for $x \in J_{i}$ and some $n_{i}>0, M\left(J_{i}\right)=K$, and

\footnotetext{
(8) The improvements are: The Benedicks-Carleson condition is not needed, the expansion factor $\rho$ in (23) can be taken arbitrarily close to $\sqrt{\lambda_{c}}$, and $c(\delta)>C|\delta|$. The flexibility on $\rho$ means we can take any $\rho \in\left(e^{\gamma}, e^{-\gamma} \sqrt{\lambda_{c}}\right)$ in Lemma 3.5. This makes (3) for $\mathscr{G}=4$ sufficient for Proposition 3.7, with no condition relating $\sigma$ and $\gamma$.

4 e SÉRIE - TOME $45-2012$ - No 6
} 
$\inf M^{\prime}(x)>1$. Let $p$ be a periodic point of $M$. Then it is easy to see that $\cup_{j} M^{-j}\{p\}$ is dense in $J$, so in particular $c$ can be approximated by preperiodic points. Now consider the restriction $M: J_{1} \cup J_{2} \rightarrow J$. Then the maximal invariant set of this map is an expanding Cantor set. Let $q$ be a point in this set with infinite orbit and whose Lyapunov exponent exists. Then $\cup_{j} M^{-j}\{q\}$ is dense in $J$, so $c$ can be approximated by non preperiodic points with well-defined and positive $\Lambda( \pm \delta)$.

We now recall the tower $\hat{f}: \hat{I} \rightarrow \hat{I}$ associated in [15] to a $\left(\lambda_{c}, H_{0}\right)$-Collet Eckmann $S$-unimodal map $f$ satisfying the Benedicks-Carleson assumption (3) for $\mathscr{G}=4$. As the (socalled subhyperbolic) case of a finite postcritical orbit is much simpler, we shall assume in this construction that this orbit is infinite. Choose $\rho$ so that

$$
e^{\gamma}<\rho<e^{-\gamma} \sqrt{\lambda_{c}}
$$

and fix ${ }^{(9)}$ two constants

$$
\frac{3}{2} \gamma<\beta_{1}<\beta_{2}<2 \gamma
$$

The tower $\hat{I}$ is the union $\hat{I}=\cup_{k \geq 0} E_{k}$ of levels $E_{k}=B_{k} \times\{k\}$ satisfying the following properties: The ground floor interval $B_{0}=\left[a_{0}, b_{0}\right]$ is just the interval $I$. For $k \geq 1$, the interval $B_{k}=\left[a_{k}, b_{k}\right]$ is such that

$$
\left[c_{k}-e^{-\beta_{2} k}, c_{k}+e^{-\beta_{2} k}\right] \subset B_{k} \subset\left[c_{k}-e^{-\beta_{1} k}, c_{k}+e^{-\beta_{1} k}\right] .
$$

(Observe that $0=c \notin B_{k}$ for all $k \geq H_{0}$.) Fix $\delta>0$ such that the Lyapunov exponents $\Lambda( \pm \delta)$ are well defined and strictly positive, so that both claims of Lemma 3.1 hold (for our present choice of $\rho$ ), and small enough so that

$$
\left|f^{j}(x)-c_{j}\right|<\min \left\{\left|c_{j}\right| e^{-\gamma j}, e^{-\beta_{2} j}\right\} \quad \text { for all } 1 \leq j \leq H_{0} \text { and }|x| \leq \delta .
$$

(Just after (31), and later on, in Section 3.2, we may need to take a smaller choice of $\delta$ still assuming that $\Lambda( \pm \delta)>0$ and that both claims of Lemma 3.1 hold.)

We may assume that the Lyapunov exponents $\Lambda\left(a_{k}\right)>0$ and $\Lambda\left(b_{k}\right)>0$ for all $k$, recalling that the set of points with a positive Lyapunov exponent has full Lebesgue measure. Let us write

$$
\{0, \pm \delta\} \cup\left\{a_{j} \mid j \geq 0\right\} \cup\left\{b_{j} \mid j \geq 0\right\}=\left\{e_{0}=c, e_{1}=\delta, e_{2}=-\delta, e_{3}, \ldots\right\} .
$$

We may and do require additionally that

$$
f^{j}\left(e_{k}\right) \neq e_{k} \text { and } f^{j}\left(e_{k}\right) \neq f^{i}\left(e_{\ell}\right) \quad \forall i, j \geq 1, k \neq \ell \geq 0 .
$$

(Indeed, (30) is a co-countable set of conditions, while the set of points $x$ with Lyapunov exponent $\Lambda(x)$ well defined and strictly positive has full Lebesgue measure, as recalled in Section 2.2.) The positivity condition on the Lyapunov exponents $\Lambda\left(e_{k}\right)(k \neq 0)$ ensures that $\alpha_{\text {cand }}\left(e_{k}\right)$ converges absolutely for each $k \geq 1$, and this will be used in the proof of Theorem 2.4.

(9) Our lower bound on $\beta_{1}$ is stronger than the one in [15] because we use some estimates in [59]. 
For $(x, k) \in E_{k}$ we set ${ }^{(10)}$

$$
\hat{f}(x, k)= \begin{cases}(f(x), k+1) & \text { if } k \geq 1 \text { and } f(x) \in B_{k+1} \\ (f(x), k+1) & \text { if } k=0 \text { and } x \in[-\delta, \delta], \\ (f(x), 0) & \text { otherwise. }\end{cases}
$$

Denoting $\pi: \hat{I} \rightarrow I$ the projection to the first factor, we have $f \circ \pi=\pi \circ \hat{f}$ on $\hat{I}$.

Define $H(\delta)$ to be the minimal $k \geq 1$ such that there exists some $x \in(-\delta, \delta)$ such that $\hat{f}^{k+1}(x, 0) \in E_{0}$. By continuity, $H(\delta)$ can be made arbitrarily large by choosing small enough $\delta$, and we assume that $H(\delta) \geq \max \left(2, H_{0}\right)$.

Having defined the tower $\hat{f}$, we next introduce notations $I_{j}, T_{i}(x)$ and $S_{i}(x)$ which will play a key part in the proof. We decompose $(-\delta, \delta) \backslash\{0\}$ as a disjoint union of intervals

$$
\begin{aligned}
& (-\delta, \delta) \backslash\{0\}=\cup_{j \geq H(\delta)} I_{j}, \quad I_{j}:=I_{j}^{+} \cup I_{j}^{-}, \\
& I_{j}^{ \pm}:=\left\{|x|<\delta, \pm x>0, \hat{f}^{\ell}(x, 0) \in E_{\ell}, 0 \leq \ell<j, \hat{f}^{j}(x, 0) \in E_{0}\right\} .
\end{aligned}
$$

(Note that $I_{j}^{ \pm}$can be empty for some $j$.) For any $k \geq H(\delta)$ both sets $J_{k}^{+}:=\cup_{H(\delta) \leq j \leq k} I_{j}^{+}$ and $J_{k}^{-}:=\cup_{H(\delta) \leq j \leq k} I_{j}^{-}$are intervals.

For each $x \in I$ we next define inductively an infinite non decreasing sequence

$$
0=S_{0}(x) \leq T_{1}(x)<S_{1}(x) \leq \cdots<S_{i}(x) \leq T_{i+1}(x)<S_{i+1}(x) \leq \cdots,
$$

with $S_{i}(x), T_{i}(x) \in \mathbb{N} \cup\{\infty\}$ as follows: Put $T_{0}(x)=S_{0}(x)=0$ for every $x \in I$. Let $i \geq 1$ and assume recursively that $S_{j}(x)$ and $T_{j}(x)$ have been defined for $j \leq i-1$. Then, we set (as usual, we put inf $\varnothing=\infty$ )

$$
T_{i}(x)=\inf \left\{j \geq S_{i-1}(x)|| f^{j}(x) \mid \leq \delta\right\} .
$$

If $T_{i}(x)=\infty$ for some $i \geq 1$ then we set $S_{i}(x)=\infty$. Otherwise, either $f^{T_{i}(x)}(x)=c$, and then we put $S_{i}(x)=\infty$, or $f^{T_{i}(x)}(x) \in I_{j}$, for some $j \geq H(\delta)$, and we put $S_{i}(x)=T_{i}(x)+j$.

Note that if $T_{i}(x)<\infty$ for some $i \geq 1$ then

$$
\begin{aligned}
& \hat{f}^{j}(x, 0) \notin E_{0}, T_{i}(x)+1 \leq j \leq S_{i}(x)-1, \\
& \hat{f}^{\ell}(x, 0) \in E_{0}, S_{i-1}(x) \leq \ell \leq T_{i}(x) .
\end{aligned}
$$

If $T_{i_{0}}(x)=\infty$ for $i_{0} \geq 1$, minimal with this property, then $\hat{f}^{\ell}(x, 0) \in E_{0}$ for all $\ell \geq S_{i_{0}-1}$ (that is, $\left|f^{\ell}(x)\right|>\delta$ for all $\ell \geq S_{i_{0}-1}$ ).

In other words, $T_{i}$ is the beginning of the $i$-th bound period and $S_{i}-1$ is the end of the $i$-th bound period, ${ }^{(11)}$ and if $S_{i}<T_{i+1}$ then $S_{i}$ is the beginning of the $i+1$-th free period (which ends when the $i+1$-th bound period starts).

In order to give a meaning to some expressions below, e.g., when $S_{i}=\infty$ or $T_{i}=\infty$, we set

$$
S_{i}-T_{i}=0 \text { if } S_{i}=T_{i}=\infty, \quad T_{i}-S_{i-1}=0 \text { if } S_{i-1}=T_{i}=\infty,
$$

and, for all $x \in I$, we set $\left(f^{\infty}\right)^{\prime}(x):=\infty$ and $f^{\infty}(x):=c_{1}$.

(10) With respect to the definition in [15], note that we replaced $(-\delta, \delta)$ by $[-\delta, \delta]$, this is not essential but convenient, e.g., in (76).

(11) Bound period refers to the fact that the orbit is bound, i.e., sufficiently exponentially close, to the postcritical orbit.

4 e SÉRIE - TOME $45-2012$ - No 6 


\subsection{Properties of the tower map}

After recalling in Lemma 3.3 and Lemma 3.4 some results of [15], we shall state in Lemma 3.5 expansion and distortion control properties of the tower map $\hat{f}$ (invoking Lemma 3.1 instead of [15, Lemma 1]). Then we shall prove two new estimates (Proposition 3.7 and its Corollary 3.8) which will play a key part in the resumation argument of Proposition 3.9.

For the sake of completeness (we shall use an estimate from the proof later on), we first recall how to obtain distortion bounds (see [15] or [59, Lemma 5.3(1)]):

Lemma 3.3 (Bounded distortion in the bound period). - Let $f$ be an S-unimodal $\left(\lambda_{c}, H_{0}\right)$-Collet-Eckmann map satisfying the Benedicks-Carleson condition (3) for $\mathscr{G}=4$, with non preperiodic critical point. Then, if $\delta$ is small enough, there exists $C>0$ such that for every $j \geq 1$, and every $k \leq j-1$, recalling (32)

$$
C^{-1} \leq \frac{\left|\left(f^{k}\right)^{\prime}(x)\right|}{\left|\left(f^{k}\right)^{\prime}(y)\right|} \leq C, \forall x, y \in U_{j}:=f\left(\{c\} \cup \bigcup_{m \geq j} I_{m}\right) .
$$

Note that $U_{j}$ is the set of points in $(-\delta, \delta) \times\{0\} \subset E_{0}$ which climb at least up to level $j-1$ before their first return to $E_{0}$.

Proof. - Recall the intervals $B_{\ell}$ from $\S 3$.1. For $1 \leq \ell \leq k \leq j-1$, pick $x_{\ell}$ and $y_{\ell}$ in $\cup_{m \geq j}\left(f^{\ell}\left(I_{m}\right)\right) \subset B_{\ell}$. We have

$$
\begin{aligned}
\prod_{\ell=1}^{k} \frac{\left|f^{\prime}\left(x_{\ell}\right)\right|}{\left|f^{\prime}\left(y_{\ell}\right)\right|} & \leq \prod_{\ell=1}^{k}\left(1+\frac{\sup \left|f^{\prime \prime}\right|}{\left|f^{\prime}\left(y_{\ell}\right)\right|}\left|x_{\ell}-y_{\ell}\right|\right) \leq \prod_{\ell=1}^{k}\left(1+C \sup \left|f^{\prime \prime}\right| \frac{\left|x_{\ell}-y_{\ell}\right|}{\left|y_{\ell}\right|}\right) \\
& \leq \prod_{\ell=1}^{\infty}\left(1+\tilde{C} C \sup \left|f^{\prime \prime}\right| e^{-\beta_{1} \ell}\right)<\infty
\end{aligned}
$$

uniformly in $m \geq j$. We used that $\left|x_{\ell}-y_{\ell}\right| \leq e^{-\beta_{1} \ell}$ and, if $\ell \geq H_{0}$, that $\left|y_{\ell}\right| \geq e^{-\gamma \ell}-e^{-\beta_{1} \ell}$ with $\beta_{1}>3 \gamma / 2$, but any summable condition would be enough here. If we choose $y_{\ell}=f^{\ell-1}(y)$ and $x_{\ell}=f^{\ell-1}(x)$ we get the upper bound in (34). If we pick $y_{\ell}=f^{\ell-1}(x)$ and $x_{\ell}=f^{\ell-1}(y)$ then we obtain the lower bound in (34).

The following upper and lower bounds from [15], about points which climb for exactly $j-1$ steps, will be used several times:

Lemma 3.4 (The $j$-bound intervals $I_{j}^{ \pm}$). - Let $f$ be an S-unimodal $\left(\lambda_{c}, H_{0}\right)$-ColletEckmann map satisfying the Benedicks-Carleson condition (3) for $\mathscr{G}=4$ and with non preperiodic critical point. Then there exist $C$ and $C_{2}$ so that for any $j \geq H(\delta)$, recalling (32), we have

$$
|x-c| \leq C e^{\frac{-3 \gamma(j-1)}{4}}\left|\left(f^{j-2}\right)^{\prime}\left(c_{1}\right)\right|^{-1 / 2}, \forall x \in I_{j},
$$

and

$$
\left|\left(f^{j}\right)^{\prime}(x)\right| \geq C_{2} e^{-\frac{\beta_{2} j}{2}}\left|\left(f^{j-1}\right)^{\prime}\left(c_{1}\right)\right|^{1 / 2}, \forall x \in I_{j}
$$

and, finally,

$$
\left|f^{\prime}(x)\right| \geq C^{-1} e^{-\gamma j}\left|\left(f^{j-1}\right)^{\prime}\left(c_{1}\right)\right|^{-1 / 2}, \forall x \in I_{j} .
$$


Proof. - If $I_{j}$ is empty, there is nothing to prove. Otherwise, our definitions and the mean value theorem imply that there exists $y$ with $f(y) \in\left[f(x), c_{1}\right]$ so that

$$
\left|\left(f^{j-2}\right)^{\prime}(f(y))\right|\left|f(x)-c_{1}\right| \leq C e^{-\beta_{1}(j-1)} .
$$

Therefore, since $\beta_{1}>3 \gamma / 2$ (recall (27)) the lower bound in (33) and the fact that $\left|f(x)-c_{1}\right| \geq$ $C^{-1}|x-c|^{2}$ yield (35).

The bound (36) follows from [15, (3.10), Proof of Lemma 2].

For (37), use (36) and that $\beta_{2}<2 \gamma$ from (27).

Recall the times $S_{i}, T_{i}$ from Subsection 3.1, for suitably small $\delta$. The following lemma gives expansion at the end of the free period $T_{i}-1$ (just before climbing the tower), at the end $S_{i}-1$ of the bound period (after falling from the tower), and during the free period (when staying at level zero):

Lemma 3.5 (Tower expansion for Benedicks-Carleson maps). - Let $f$ be an S-unimodal $\left(\lambda_{c}, H_{0}\right)$-Collet-Eckmann map satisfying the Benedicks-Carleson condition (3) for $\mathscr{G}=4$, with non preperiodic critical point, and let $\rho$ satisfy (26). For every small enough $\delta_{0}>0$, if $\delta<\delta_{0}$, $\sigma>1, C_{1}=C_{1}(\rho) \in(0,1]$, and $c(\delta)>0$ are as in Lemma 3.1, letting $S_{i}(x)$ and $T_{i}(x)$ be the times associated to the tower for $\delta$, then

$$
\left|\left(f^{S_{i}(x)}\right)^{\prime}(x)\right| \geq \rho^{S_{i}(x)}, \quad\left|\left(f^{T_{i}(x)}\right)^{\prime}(x)\right| \geq C_{1} \rho^{T_{i}(x)}, \quad \forall x \in I, \quad \forall i \geq 0,
$$

and

$$
\left|\left(f^{S_{i}(x)+j}\right)^{\prime}(x)\right| \geq c(\delta) \rho^{S_{i}(x)} \sigma^{j}, \quad \forall x \in I, \quad \forall i \geq 0, \forall 0 \leq j<T_{i+1}(x)-S_{i}(x) .
$$

REMARK 3.6. - An immediate consequence of Lemma 3.5 is that, for every $x$ such that $f^{n}(x) \neq c$, for every $n$, we have $\lim \sup _{n}\left|\left(f^{n}\right)^{\prime}(x)\right|^{1 / n} \geq \xi>1$, where $\xi=\min (\rho, \sigma)$.

Proof of Lemma 3.5. - Choose $\delta<\delta_{0}$ as in the second claim of Lemma 3.1, small enough so that

$$
C_{1} C_{2} \cdot e^{-\frac{\beta_{2} j}{2}} \lambda_{c}^{\frac{j-1}{2}} \geq \rho^{j}, \quad \forall j \geq H(\delta) .
$$

Let now $x \in I$. Recall that for any $\ell \geq 1$, the definitions imply $f^{S_{\ell-1}(x)+k}(x) \in I \backslash[-\delta, \delta]$ for all $0 \leq k<T_{\ell}(x)-S_{\ell-1}(x)$ and $f^{T_{\ell}(x)}(x) \in I_{j}$ with $j=S_{\ell}(x)-T_{\ell}(x) \geq H(\delta)$. Therefore, the second claim of Lemma 3.1 and (36) give for all $i \geq 0$

$$
\left|\left(f^{S_{i}}\right)^{\prime}(x)\right|=\prod_{\ell=1}^{i}\left|\left(f^{S_{\ell}(x)-T_{\ell}(x)}\right)^{\prime}\left(f^{T_{\ell}(x)} x\right)\right|\left|\left(f^{T_{\ell}(x)-S_{\ell-1}(x)}\right)^{\prime}\left(f^{S_{\ell-1}(x)} x\right)\right| \geq \rho^{S_{i}(x)},
$$

and

$$
\left|\left(f^{T_{i}}\right)^{\prime}(x)\right|=\left|\left(f^{T_{i}(x)-S_{i-1}(x)}\right)^{\prime}\left(f^{S_{i-1}(x)} x\right)\right|\left|\left(f^{S_{i}}\right)^{\prime}(x)\right| \geq C_{1} \rho^{T_{i}(x)-S_{i}(x)} \rho^{S_{i}(x)} .
$$

Using in addition the first claim of Lemma 3.1, we get, for $0 \leq j \leq T_{i+1}(x)-S_{i}(x)$,

$$
\left|\left(f^{S_{i}(x)+j}\right)^{\prime}(x)\right|=\left|\left(f^{j}\right)^{\prime}\left(f^{S_{i}(x)}(x)\right)\right|\left|\left(f^{S_{i}}\right)^{\prime}(x)\right| \geq c(\delta) \sigma^{j} \rho^{S_{i}(x)} .
$$

4 e SÉRIE - TOME $45-2012-$ NNo $^{\circ}$ 
The information on the tower will allow us to prove the next proposition, which is a crucial ingredient ${ }^{(12)}$ to show that $\alpha_{\text {cand }}$ can be resummed to a bounded function (Proposition 3.9 and Definition 3.10):

Proposition 3.7 (Key estimate for Benedicks Carleson maps). - Let $f$ be an S-unimodal $\left(\lambda_{c}, H_{0}\right)$-Collet-Eckmann map satisfying the Benedicks-Carleson condition (3) for $\mathscr{G}=4$, with non preperiodic critical point. There exists $C>0$ such that for every $j \geq 0$ we have

$$
\sum_{k=j+1}^{\infty} \frac{1}{\left|\left(f^{k-j}\right)^{\prime}\left(f^{j}\left(c_{1}\right)\right)\right|} \leq C e^{\gamma j}
$$

The proof shows that $C=O\left((c(\delta))^{-1}\right)$, where $\delta$ is the parameter used in the construction of the tower and $c(\delta)$ is given by Lemma 3.1. More importantly, the proposition implies that $\left|\alpha_{\text {cand }}\left(c_{j}\right)\right| \leq C \sup |v| e^{\gamma j}$. This bound is of course not uniform in $j$, but it will act as a bootstrap for the proof of the Proposition 3.9 which performs the resumation.

Proof. - Fix $j \geq 1$. Since the summands are all positive, we may (and shall) group them in a convenient way, using the times $T_{i}:=T_{i}\left(c_{j+1}\right)$ and $S_{i}:=S_{i}\left(c_{j+1}\right)$ defined in the tower construction for a small enough $\delta$. We have

$$
\begin{aligned}
& \sum_{k=j+1}^{\infty} \frac{1}{\left|\left(f^{k-j}\right)^{\prime}\left(f^{j}\left(c_{1}\right)\right)\right|} \\
& \quad=\sum_{i=0}^{\infty} \frac{1}{\left|\left(f^{S_{i}}\right)^{\prime}\left(c_{j+1}\right)\right|} u_{T_{i+1}-S_{i}}\left(f^{S_{i}}\left(c_{j+1}\right)\right)+\sum_{i=1}^{\infty} \frac{1}{\left|\left(f^{T_{i}}\right)^{\prime}\left(c_{j+1}\right)\right|} u_{S_{i}-T_{i}}\left(f^{T_{i}}\left(c_{j+1}\right)\right)
\end{aligned}
$$

where we use the notation

$$
u_{n}(y)=\sum_{\ell=1}^{n} \frac{1}{\left|\left(f^{\ell}\right)^{\prime}(y)\right|} .
$$

(In particular $u_{0} \equiv 0$.) Since $T_{i+1}-S_{i}=T_{1}\left(f^{S_{i}}\left(c_{j+1}\right)\right)$, Lemma 3.5 implies

$$
u_{T_{i+1}-S_{i}}\left(f^{S_{i}}\left(c_{j+1}\right)\right) \leq \frac{C}{c(\delta)\left(1-\sigma^{-1}\right)}
$$

(in particular the series converges if $\left.n=T_{i+1}-S_{i}=\infty\right)$. Since $f^{\prime \prime}(c) \neq 0$, the BenedicksCarleson assumption (3) for $\mathscr{G}=4$ implies for all $i$

$$
\left|f^{\prime}\left(f^{T_{i}}\left(c_{j+1}\right)\right)\right|=\left|f^{\prime}\left(f^{T_{i}+j}\left(c_{1}\right)\right)\right| \geq C^{-1} e^{-\gamma\left(T_{i}+j\right)} .
$$

Therefore, the bounded distortion estimate (34) in the proof of Lemma 3.3 gives, together with the Collet-Eckmann assumption, ${ }^{(13)}$

$$
u_{S_{i}-T_{i}}\left(f^{T_{i}}\left(c_{j+1}\right)\right) \leq \frac{1}{\left|f^{\prime}\left(f^{T_{i}}\left(c_{j+1}\right)\right)\right|} \sum_{\ell=0}^{\infty} \frac{C}{\left|\left(f^{\ell}\right)^{\prime}\left(c_{1}\right)\right|} \leq \frac{C^{3}}{\left(1-\lambda_{c}^{-1}\right)} e^{\gamma\left(T_{i}+j\right)} .
$$

\footnotetext{
(12) Proposition 3.7 will also be used in an essential way in the proof of Theorem 2.13, in particular in the proof of Lemma 4.1, and also in Lemma 4.12.

(13) The constant $C$ above depends on $\left[\sup _{1 \leq j<H_{0}} \lambda_{c} /\left|\left(f^{j}\right)^{\prime}\left(c_{1}\right)\right|^{1 / j}\right]^{H_{0}}$. By Lemma 5.8 , this expression is uniform for suitable families $f_{t}$.
} 
By Lemma 3.5 we have $\left|\left(f^{S_{i}}\right)^{\prime}\left(c_{j+1}\right)\right| \geq C \rho^{S_{i}}$ and $\left|\left(f^{T_{i}}\right)^{\prime}\left(c_{j+1}\right)\right| \geq C_{1} \rho^{T_{i}}$. Therefore, there exist constants $K_{1}(\delta), K_{2}(\delta)$ so that

$$
\sum_{k=j+1}^{\infty} \frac{1}{\left|\left(f^{k-j}\right)^{\prime}\left(f^{j}\left(c_{1}\right)\right)\right|} \leq K_{1}(\delta) e^{\gamma j}\left[\sum_{i=0}^{\infty} \rho^{-S_{i}}+\sum_{i=1}^{\infty} \rho^{-T_{i}} e^{\gamma T_{i}}\right] \leq K_{2}(\delta) e^{\gamma j}
$$

(We used that $\rho>e^{\gamma}$.)

We end this section of preparations by a consequence of Proposition 3.7 which will also be needed in our resumation Proposition 3.9:

Corollary 3.8. - Let $f$ be an $S$-unimodal $\left(\lambda_{c}, H_{0}\right)$-Collet-Eckmann map satisfying the Benedicks-Carleson condition (3) for $\mathscr{G}=4$, with non preperiodic critical point. Then there exists $C$ so that for any $j \geq 1$

$$
\frac{1}{\left|f^{\prime}(x)\right|} \sum_{k=1}^{j-1}\left|\frac{1}{\left|\left(f^{k}\right)^{\prime}\left(c_{1}\right)\right|}-\frac{1}{\left|\left(f^{k}\right)^{\prime}(f(x))\right|}\right| \leq C \frac{e^{5 \gamma j / 4}}{\left|f^{j-2}\left(c_{1}\right)\right|^{1 / 2}}, \forall x \in I_{j} .
$$

Proof. - For any $j \geq 1, x \in I_{j}$, and $1 \leq k<j$ we get by using (34) from the proof of Lemma 3.3 that

$$
\begin{aligned}
& \left|\frac{1}{\left(f^{k}\right)^{\prime}(f(x))}-\frac{1}{\left(f^{k}\right)^{\prime}\left(c_{1}\right)}\right| \\
& \quad \leq \sum_{n=0}^{k-1}\left|\frac{1}{f^{\prime}\left(f^{n}(f(x))\right)}-\frac{1}{f^{\prime}\left(f^{n}\left(c_{1}\right)\right)}\right| \prod_{\ell=0}^{n-1} \frac{1}{\left|f^{\prime}\left(f^{\ell}\left(c_{1}\right)\right)\right|} \prod_{i=n+1}^{k-1} \frac{1}{\left|f^{\prime}\left(f^{i}(f(x))\right)\right|} \\
& \quad \leq \sum_{n=0}^{k-1} \sup _{y \in f^{n}\left[f(x), c_{1}\right]} \frac{\left|f^{\prime \prime}(y)\right|}{\left|f^{\prime}(y)\right|^{2}}\left|f^{n}(f(x))-f^{n}\left(c_{1}\right)\right| \prod_{\ell=0}^{n-1} \frac{1}{\mid f^{\prime}\left(f^{\ell}\left(c_{1}\right) \mid\right.} \prod_{i=n+1}^{k-1} \frac{1}{\left|f^{\prime}\left(f^{i}(f(x))\right)\right|} \\
& \quad \leq \sum_{n=0}^{k-1} \sup _{y \in f^{n}\left[f(x), c_{1}\right]} \frac{\left|f^{\prime \prime}(y)\right|}{\left|f^{\prime}(y)\right|^{2}} \sup _{z \in\left[f(x), c_{1}\right]}\left|\left(f^{n}\right)^{\prime}(z)\right|\left|f(x)-c_{1}\right| \\
& \quad \leq \prod_{\ell=0}^{n-1} \frac{1}{\mid f^{\prime}\left(f^{\ell}\left(c_{1}\right) \mid\right.} \prod_{i=n+1}^{k-1} \frac{1}{\left|f^{\prime}\left(f^{i}(f(x))\right)\right|} \\
& \quad \leq \frac{C\left|f(x)-c_{1}\right| \sum_{n=0}^{k} \sup _{y \in f^{n}\left[f(x), c_{1}\right]} \frac{\left|f^{\prime \prime}(y)\right|}{\left|f^{\prime}(y)\right|} \frac{\left|\left(f^{n}\right)^{\prime}\left(c_{1}\right)\right|}{\left|\left(f^{k}\right)^{\prime}\left(c_{1}\right)\right|}}{1-e^{-\gamma / 2}\left|f(x)-c_{1}\right| \sum_{n=0}^{k-1} e^{\gamma n} \frac{1}{\left|\left(f^{k-n}\right)^{\prime}\left(f^{n}\left(c_{1}\right)\right)\right|}}
\end{aligned}
$$

(In the last inequality, we used that $f^{n}\left[f(x), c_{1}\right] \subset B_{n+1}$, and that the Benedicks-Carleson assumption (3) for $\mathscr{G}=4$ implies $|y-c|>e^{-\gamma(n+1)}-e^{-\beta_{1}(n+1)}$ for $y \in B_{n+1}$, together with (27).)

$4^{\text {e }}$ SÉRIE - TOME $45-2012-\mathrm{N}^{\circ} 6$ 
Therefore, for any $j \geq 1$ and $x \in I_{j}$, since $\left|f(x)-c_{1}\right| \leq C|x-c|^{2}$, Proposition 3.7 implies

$$
\begin{aligned}
& \frac{1}{\left|f^{\prime}(x)\right|} \sum_{k=0}^{j-1}\left|\frac{1}{\left(f^{k}\right)^{\prime}(f(x))}-\frac{1}{\left(f^{k}\right)^{\prime}\left(c_{1}\right)}\right| \\
& \quad \leq C|x-c| \sum_{k=0}^{j-1} \sum_{n=0}^{k} e^{\gamma n} \frac{1}{\left|\left(f^{k-n}\right)^{\prime}\left(f^{n}\left(c_{1}\right)\right)\right|} \\
& \quad \leq C|x-c| \sum_{n=0}^{j-1} e^{\gamma n} \sum_{i=0}^{j-n-1} \frac{1}{\left|\left(f^{i}\right)^{\prime}\left(f^{n}\left(c_{1}\right)\right)\right|} \\
& \quad \leq C|x-c| \sum_{n=0}^{j-1} e^{\gamma n} \sum_{i=0}^{\infty} \frac{1}{\left|\left(f^{i}\right)^{\prime}\left(f^{n}\left(c_{1}\right)\right)\right|} \\
& \quad \stackrel{(39)}{\leq} C|x-c| \sum_{n=0}^{j-1} e^{2 \gamma n} \leq C|x-c| e^{2 \gamma j} \leq C \frac{e^{-3 \gamma(j-1) / 4}}{\left|\left(f^{j-2}\right)^{\prime}\left(c_{1}\right)\right|^{1 / 2}} e^{2 \gamma j},
\end{aligned}
$$

where we used (35) from Lemma 3.4 in the last inequality.

\subsection{Resumation: Definition and boundedness of $\alpha$ for horizontal $v$}

Proposition 3.9 is the heart of the proof of Theorem 2.4. This is where we define the dynamical resumation for the series $\alpha_{\text {cand }}$, under a horizontality condition.

Proposition 3.9 (Resumation). - Let $f$ be an S-unimodal $\left(\lambda_{c}, H_{0}\right)$-Collet-Eckmann map satisfying the Benedicks-Carleson condition (3) for $\mathscr{G}=4$, with non preperiodic critical point. Let $v=X \circ f$, for $X$ a Lipschitz function, and assume that $v$ satisfies the horizontality condition (10) for $f$.

If $\delta$ is small enough, then for every $x \in I$, letting $T_{i}=T_{i}(x)$ and $S_{i}=S_{i}(x)$ be the times associated to the tower for $\delta$, the following series converges:

$$
\sum_{i=1}^{\infty}\left(\frac{1}{\left|\left(f^{T_{i}}\right)^{\prime}(x)\right|}\left|w_{S_{i}-T_{i}}\left(f^{T_{i}}(x)\right)\right|+\frac{1}{\left|\left(f^{S_{i-1}}\right)^{\prime}(x)\right|}\left|w_{T_{i}-S_{i-1}}\left(f^{S_{i-1}}(x)\right)\right|\right),
$$

where $w_{\infty}(c)=0$ and ${ }^{(14)}$

$$
w_{n}(y):=\sum_{\ell=0}^{n-1} \frac{v\left(f^{\ell}(y)\right)}{\left(f^{\ell+1}\right)^{\prime}(y)}, \quad y \neq c, n \in \mathbb{Z}_{+} \cup\{\infty\} .
$$

Moreover the sum of the series (42) is bounded uniformly in $x \in I$.

The proposition allows us to give the following definition:

Definition 3.10. - We define $\alpha(x)$ for any $x \in I$ by

$$
\alpha(x)=-\sum_{i=1}^{\infty}\left(\frac{1}{\left(f^{T_{i}}\right)^{\prime}(x)} w_{S_{i}-T_{i}}\left(f^{T_{i}}(x)\right)+\frac{1}{\left(f^{S_{i-1}}\right)^{\prime}(x)} w_{T_{i}-S_{i-1}}\left(f^{S_{i-1}}(x)\right)\right) .
$$

(14) In particular, we claim that $w_{n}(y)$ converges if $n=S_{i}-T_{i}=\infty$ and $y=f^{T_{i}}(x) \neq c$, or if $n=T_{i}-S_{i-1}=\infty$ and $y=f^{S_{i-1}}(x)$. 
If the formal series (8) is absolutely convergent at $x$, then (43) is just the sum of this series. If $f^{j}(x)=c$ for some $j \geq 0$, minimal with this property, then our notation ensures that (43) is just the finite sum (9). In both these cases, $\alpha(x)=\alpha_{\text {cand }}(x)$.

Proof of Proposition 3.9.. - Choose $\delta$ small enough, as in Lemma 3.5, and $C_{1}(\rho), \sigma>1$, and $c(\delta)$ from Lemma 3.1.

For any $i \geq 1$ so that $S_{i-1}<\infty$, since $T_{i}-S_{i-1}=T_{1}\left(f^{S_{i-1}}(x)\right)$, Lemma 3.5 implies

$$
\begin{aligned}
\left|w_{T_{i}-S_{i-1}}\left(f^{S_{i-1}}(x)\right)\right| & \leq \sum_{\ell=0}^{T_{i}-S_{i-1}-1}\left|\frac{v\left(f^{\ell}\left(f^{S_{i-1}}(x)\right)\right)}{\left(f^{\ell+1}\right)^{\prime}\left(f^{S_{i-1}}(x)\right)}\right| \\
& \leq \frac{C}{c(\delta)\left(1-\sigma^{-1}\right)} \sup |v|, \quad \forall i \geq 0 .
\end{aligned}
$$

(Note that $T_{i}=\infty$ is allowed in the previous estimate.)

If $S_{i}=\infty$ then either $f^{T_{i}}(x)=c$ or $T_{i}=\infty$; in both cases $w_{S_{i}-T_{i}}\left(f^{T_{i}}(x)\right)=0$. Next, we claim that whenever $S_{i} \neq \infty$, we have,

$$
\left|w_{S_{i}-T_{i}}\left(f^{T_{i}}(x)\right)\right| \leq C \max \{\sup |v|, \operatorname{Lip} X\} \frac{e^{2 \gamma\left(S_{i}-T_{i}\right)}}{\left|\left(f^{S_{i}-T_{i}-1}\right)^{\prime}\left(c_{1}\right)\right|^{1 / 2}}, \forall i \geq 0 .
$$

We shall prove (45), which requires horizontality as well as the key Proposition 3.7 and its Corollary 3.8, at the end of this proof.

Putting together (44) and (45), and recalling Lemma 3.5, we find the following upper bound for (42) :

$$
\sum_{i=1}^{\infty} \frac{C \rho^{-S_{i-1}}}{c(\delta)\left(1-\sigma^{-1}\right)} \sup |v|+C \frac{\rho^{-T_{i}}}{C_{1}(\rho)} \max (\sup |v|, \operatorname{Lip} X) \frac{e^{2 \gamma\left(S_{i}-T_{i}\right)}}{\left|\left(f^{S_{i}-T_{i}-1}\right)^{\prime}\left(c_{1}\right)\right|^{1 / 2}} .
$$

Using again (3) for $\mathscr{G}=4$, we are done.

It remains to prove (45). The definitions imply $f^{T_{i}}(x) \in I_{j}$ for $j=S_{i}-T_{i}$, for all $i \geq 1$. So it suffices to show that

$$
\left|w_{j}(y)\right| \leq C \max (\sup |v|, \operatorname{Lip} X) \frac{e^{2 \gamma j}}{\left|\left(f^{j-1}\right)^{\prime}\left(c_{1}\right)\right|^{1 / 2}}, \quad \forall y \in I_{j} .
$$

We shall use the decomposition

$$
\begin{gathered}
\left|w_{j}(y)\right| \leq \underbrace{\frac{1}{\left|f^{\prime}(y)\right|}\left|\sum_{k=0}^{j-1} \frac{v\left(c_{k}\right)}{\left(f^{k}\right)^{\prime}\left(c_{1}\right)}\right|}_{I}+\underbrace{\frac{1}{\left|f^{\prime}(y)\right|} \sum_{k=0}^{j-1}\left|v\left(c_{k}\right)\right| \frac{1}{\left(f^{k}\right)^{\prime}(f(y))}-\frac{1}{\left(f^{k}\right)^{\prime}\left(c_{1}\right)} \mid}_{I I} \\
+\underbrace{\frac{1}{\left|f^{\prime}(y)\right|} \sum_{k=0}^{j-1}\left|\frac{v\left(f^{k}(y)\right)-v\left(c_{k}\right)}{\left(f^{k}\right)^{\prime}(y)}\right|}_{I I I} .
\end{gathered}
$$

We first consider $I$. By the horizontality condition (10) for $v$, we have

$$
\sum_{k=0}^{j-1} \frac{v\left(c_{k}\right)}{\left(f^{k}\right)^{\prime}\left(c_{1}\right)}+\frac{1}{\left(f^{j}\right)^{\prime}\left(c_{1}\right)} \sum_{k=j}^{\infty} \frac{v\left(c_{k}\right)}{\left(f^{k-j}\right)^{\prime}\left(c_{j+1}\right)}=0 .
$$

$4^{\text {e }}$ SÉRIE - TOME $45-2012$ - No 6 
Therefore, (37) in Lemma 3.4 and Proposition 3.7 imply

$$
\begin{aligned}
I & =\frac{1}{\left|f^{\prime}(y)\right|} \frac{1}{\left|\left(f^{j}\right)^{\prime}\left(c_{1}\right)\right|}\left|\sum_{k=j}^{\infty} \frac{v\left(c_{k}\right)}{\left(f^{k-j}\right)^{\prime}\left(c_{j+1}\right)}\right| \\
& \leq C e^{\gamma j}\left|\left(f^{j-1}\right)^{\prime}\left(c_{1}\right)\right|^{1 / 2} \frac{1}{\left|\left(f^{j}\right)^{\prime}\left(c_{1}\right)\right|} \sup |v| e^{\gamma j} \\
& \leq C \sup |v| \frac{e^{2 \gamma j}}{\left|\left(f^{j-1}\right)^{\prime}\left(c_{1}\right)\right|^{1 / 2}} .
\end{aligned}
$$

Next, by Corollary 3.8 we find

$$
\begin{aligned}
I I & \leq \sup |v| \frac{1}{\left|f^{\prime}(y)\right|} \sum_{k=0}^{j-1}\left|\frac{1}{\left(f^{k}\right)^{\prime}(f(y))}-\frac{1}{\left(f^{k}\right)^{\prime}\left(c_{1}\right)}\right| \\
& \leq C \sup |v| \frac{e^{5 \gamma j / 4}}{\left|\left(f^{j-2}\right)^{\prime}\left(c_{1}\right)\right|^{1 / 2}} .
\end{aligned}
$$

Recalling our assumption $v=X \circ f$, we consider now

$$
I I I=\frac{1}{\left|f^{\prime}(y)\right|} \sum_{k=0}^{j-1}\left|\frac{X\left(f^{k+1}(y)\right)-X\left(c_{k+1}\right)}{\left(f^{k}\right)^{\prime}(y)}\right| .
$$

Since $X$ is Lipschitz and $f$ is $C^{1}$, for any $0 \leq k \leq j-1$ there exists $z$ between $c_{1}$ and $f(y)$ so that

$$
\begin{aligned}
\left|X\left(f^{k+1}(y)\right)-X\left(f^{k+1}(c)\right)\right| & \leq \operatorname{Lip}(X)\left|f^{k}(f(y))-f^{k}\left(c_{1}\right)\right| \\
& \leq \operatorname{Lip}(X)\left|f(y)-c_{1}\right|\left|\left(f^{k}\right)^{\prime}(z)\right| .
\end{aligned}
$$

Then (51), together with (33) from the proof of Lemma 3.3 (recall $k \leq j-1$ and $y \in I_{j}$ ), imply

$$
\begin{aligned}
\frac{\left|X\left(f^{k+1}(y)\right)-X\left(f^{k+1}(c)\right)\right|}{\left|\left(f^{k}\right)^{\prime}\left(c_{1}\right)\right|} & \leq \operatorname{Lip} X\left|f(y)-c_{1}\right| \sup _{z \in\left[f(y), c_{1}\right]} \frac{\left|\left(f^{k}\right)^{\prime}(z)\right|}{\left(f^{k}\right)^{\prime}\left(c_{1}\right)} \\
& \leq C \operatorname{Lip} X\left|f(y)-c_{1}\right| \\
& \leq C \operatorname{Lip} X|y-c| \sup _{z \in[c, y]}\left|f^{\prime}(z)\right| .
\end{aligned}
$$

(Recall that in this proof $C$ denotes a universal constant, which can however vary.) Since $\left|f^{\prime}(y)\right| \geq C|y-c|$ and $\left|f^{\prime}(z)\right| \leq C|z-c|$, the bound (35) in Lemma 3.4 gives

$$
\begin{aligned}
I I I & \leq C \frac{j}{\left|f^{\prime}(y)\right|} \operatorname{Lip} X|y-c| \sup _{z \in[c, y]}\left|f^{\prime}(z)\right| \leq C j \operatorname{Lip} X \sup _{z \in[c, y]}|z-c| \\
& \leq C j \operatorname{Lip} X e^{-\frac{3 \gamma(j-1)}{4}}\left|\left(f^{j-2}\right)^{\prime}\left(c_{1}\right)\right|^{-1 / 2} \leq C \operatorname{Lip} X \frac{1}{\left|\left(f^{j-2}\right)^{\prime}\left(c_{1}\right)\right|^{1 / 2}} .
\end{aligned}
$$

Putting (48), (49), and (52) together, we get (46). 


\subsection{Proof of Theorem 2.4: Continuity of $\alpha$ and checking the TCE}

We prove that Proposition 3.9 implies Theorem 2.4:

Proof of Theorem 2.4. - The so-called subhyperbolic case when the critical point is preperiodic is easier and left to the reader. For small enough $\delta$ (recall Sections 3.1 and 3.2), we construct a tower map and associated times $T_{i}(x)$ and $S_{i}(x)$.

To show the uniqueness statement for bounded $v$, suppose that $\beta: I \rightarrow \mathbb{C}$ is a bounded function such that $v=\beta \circ f-f^{\prime} \cdot \beta$ on $I$. It is easy to see that for every $x$ and $n \geq 1$ such that $\left(f^{n}\right)^{\prime}(x) \neq 0$ we have

$$
\beta(x)=-\sum_{i=0}^{n-1} \frac{v\left(f^{i}(x)\right)}{\left(f^{i+1}\right)^{\prime}(x)}+\frac{\beta\left(f^{n}(x)\right)}{\left(f^{n}\right)^{\prime}(x)} .
$$

If $f^{n}(x) \neq c$ for every $n$, Remark 3.6 implies that $\limsup _{n}\left|\left(f^{n}\right)^{\prime}(x)\right|=\infty$, so that there exists ${ }^{(15)} n_{i}(x) \rightarrow_{i} \infty$ with $\lim _{i}\left|\left(f^{n_{i}(x)}\right)^{\prime}(x)\right|=\infty$. Since $\beta$ is bounded, it follows from (53) that

$$
\beta(x)=-\lim _{i \rightarrow \infty} \sum_{j=0}^{n_{i}} \frac{v\left(f^{j}(x)\right)}{\left(f^{j+1}\right)^{\prime}(x)} .
$$

This proves that $\beta$ is uniquely defined on $\left\{x \mid f^{n}(x) \neq c, \forall n\right\}$. In particular, $\beta\left(c_{1}\right)=$ $\alpha_{\text {cand }}\left(c_{1}\right)$, so that $v$ is horizontal (using the TCE, that $f^{\prime}(c)=0$, and that $\beta$ is bounded).

Now, if $f^{i}(x) \neq c$ for $0 \leq i<n$ and $f^{n}(x)=c$, then (53) and $\beta(c)=0$ give

$$
\beta(x)=-\sum_{i=0}^{n-1} \frac{v\left(f^{i}(x)\right)}{\left(f^{i+1}\right)^{\prime}(x)} .
$$

Therefore, $\beta(x)=\alpha_{\text {cand }}(x)$. This ends the proof of uniqueness.

From now on, we assume that $v=X \circ f$ with $X$ Lipschitz. If $v$ is horizontal, Proposition 3.9 implies that the function $\alpha(x)$ defined by the series (43) is bounded uniformly in $x \in I$. It remains to show that $\alpha$ is continuous and satisfies the TCE.

The definitions easily imply that for every $x \in I$ and all $i \geq 1$ the following limits exist:

$$
\begin{array}{ll}
S_{i}^{+}(x)=\lim _{y \rightarrow x^{+}} S_{i}(y), & S_{i}^{-}(x)=\lim _{y \rightarrow x^{-}} S_{i}(y), \\
T_{i}^{+}(x)=\lim _{y \rightarrow x^{+}} T_{i}(y), & T_{i}^{-}(x)=\lim _{y \rightarrow x^{-}} T_{i}(y) .
\end{array}
$$

Writing $T_{i}^{ \pm}$and $S_{i}^{ \pm}$for $T_{i}^{ \pm}(x)$ and $S_{i}^{ \pm}(x)$, define

$$
\alpha^{ \pm}(x):=-\sum_{i=1}^{\infty}\left(\frac{1}{\left(f^{T_{i}^{ \pm}}\right)^{\prime}(x)} w_{S_{i}^{ \pm}-T_{i}^{ \pm}}\left(f^{T_{i}^{ \pm}}(x)\right)+\frac{1}{\left(f^{\left.S_{i-1}^{ \pm}\right)^{\prime}}(x)\right.} w_{T_{i}^{ \pm}-S_{i-1}^{ \pm}}\left(f^{S_{i-1}^{ \pm}}(x)\right)\right) .
$$

We claim that for every $x \in I$ we have

$$
\lim _{y \rightarrow x^{+}} \alpha(y)=\alpha^{+}(x), \text { and } \lim _{y \rightarrow x^{-}} \alpha(y)=\alpha^{-}(x) .
$$

We shall show (56) at the end of the proof of this theorem.

Let now $\&$ be the set of $x \in I$ so that there exists $\ell \geq 0$ with $\hat{f}^{\ell}(x, 0) \in \partial E_{k}$ for some $k \geq 1$, or $\hat{f}^{\ell}(x, 0)=( \pm \delta, 0)$, or $\hat{f}^{\ell}(x, 0)=(c, 0)$. Clearly, if $x \notin \ell$, then $S_{i}(x)=S_{i}^{+}(x)=S_{i}^{-}(x)$

$\overline{\text { (15) Note that if } T_{i}}(x)<\infty$ for all $i$, we can take $n_{i}(x)=T_{i}(x)$. 
and $T_{i}(x)=T_{i}^{+}(x)=T_{i}^{-}(x)$, for every $i \geq 1$. Consequently $\alpha$ is continuous at $x \notin \&$. If $x \in \&$ but $\hat{f}^{\ell}(x, 0) \neq(c, 0)$ for all $\ell \geq 0$, then the conditions on $\left\{e_{k}\right\}$ in Section 3.1 imply that the series (8) converges absolutely at $x$. If $\hat{f}^{\ell}(x, 0)=(c, 0)$ for some $\ell \geq 0$, then $\alpha(x)$ is the finite sum (9). Let now $x \in \varnothing$. The three series, or finite sums, which define $\alpha(x), \alpha^{+}(x)$, and $\alpha^{-}(x)$ are obtained by grouping together in different ways the terms of the absolutely convergent series (8), or of the sum (9). Therefore, $\alpha(x)=\alpha^{+}(x)=\alpha^{-}(x)$, and (56) implies that $\alpha$ is continuous at $x$.

To show that $\alpha$ satisfies the twisted cohomological equation, note that if $x$ is a repelling periodic point then $f^{\ell}(x) \neq c$ for all $\ell$, and the series (8) is absolutely convergent at $x$. Therefore, this series coincides with $\alpha(x)$. In particular one can easily check that $v(x)=\alpha(f(x))-f^{\prime}(x) \alpha(x)$ for repelling periodic points $x$. Since all periodic points of a Collet-Eckmann map are repelling, since the set of periodic points is dense, and since $\alpha$ is continuous, it follows that $\alpha$ satisfies the twisted cohomological equation everywhere.

The fact that $\alpha(x)=\alpha_{\text {cand }}(x)$ for all $x$ so that $f^{j}(x)=c$ or such that the series $\alpha_{\text {cand }}(x)$ converges absolutely follows from the remark after Definition 3.10.

It remains to prove (56). We shall consider the limit as $y$ approaches $x$ from above (the proof of the other one-sided limit is identical).

Before we start, note that for any $\epsilon>0$, the uniform constants and exponential rates in the proof of Proposition 3.9 (see (44), where $T_{i}=\infty$ is allowed, and (45), (38)) imply that there is $n_{0}=n_{0}(\epsilon) \geq 1$ such that for all $x \in I$

$$
\sum_{i: T_{i} \geq n_{0}} \frac{1}{\left|\left(f^{T_{i}}\right)^{\prime}(x)\right|}\left|w_{S_{i}-T_{i}}\left(f^{T_{i}}(x)\right)\right|+\sum_{i: S_{i} \geq n_{0}} \frac{1}{\mid\left(f^{S_{i}}\right)^{\prime}(x)}\left|w_{T_{i+1}-S_{i}}\left(f^{S_{i}}(x)\right)\right|<\frac{\epsilon}{4} .
$$

Fix $x \in I$, and let $T_{i}=T_{i}(x), S_{i}=S_{i}(x), T_{i}^{+}=T_{i}^{+}(x), S_{i}^{+}=S_{i}^{+}(x)$. There are three cases to consider to prove (56) when $y \rightarrow x^{+}$. The first case occurs when $T_{i}<\infty$ and $S_{i}<\infty$ for every $i$. This means that the forward $\hat{f}$-orbit of $x$ never hits the critical point $(c, 0)$ and never gets trapped inside the base $E_{0}$. Then observe that there exists $\epsilon_{1}>0$ such that if $x<y<x+\epsilon_{1}$ then, for every $i$ so that $S_{i}^{+}<n_{0}$, we have $S_{i}(y)=S_{i}^{+}$, and for every $i$ so that $T_{i}^{+}(x)<n_{0}$, we have $T_{i}(y)=T_{i}^{+}$. Clearly, for any $n_{0} \geq 1$, the function

$$
\begin{aligned}
& \tilde{\alpha}(y)=\tilde{\alpha}_{n_{0}}(y)=-\sum_{i: T_{i}^{+}<n_{0}} \frac{1}{\left(f^{T_{i}^{+}}\right)^{\prime}(y)} w_{S_{i}^{+}-T_{i}^{+}}\left(f^{T_{i}^{+}}(y)\right) \\
&-\sum_{i: S_{i}^{+}<n_{0}} \frac{1}{\left(f^{S_{i}^{+}}\right)^{\prime}(y)} w_{T_{i+1}^{+}(x)-S_{i}^{+}}\left(f^{S_{i}^{+}}(y)\right)
\end{aligned}
$$

is continuous on $\left[x, x+\epsilon_{1}\right.$ ). So for any $\epsilon>0$ there exists $0<\epsilon_{2}<\epsilon_{1}$ (depending also on $n_{0}$ ) such that if $x \leq y<x+\epsilon_{2}$ then $|\tilde{\alpha}(y)-\tilde{\alpha}(x)|<\epsilon / 2$. Clearly, $\tilde{\alpha}(x)$ is just the $n_{0}$-truncation of $\alpha^{+}(x)$ while the observation above implies that $\tilde{\alpha}(y)$ is the $n_{0}$-truncation of $\alpha(y)$. Thus, taking $n_{0}(\epsilon)$ as in (57), we get that

$$
\left|\alpha(y)-\alpha^{+}(x)\right|<|\tilde{\alpha}(y)-\tilde{\alpha}(x)|+\frac{2 \epsilon}{4}<\epsilon, \quad \forall y \in\left[x, x+\epsilon_{2}\right) .
$$

The second case occurs when the forward $\hat{f}$-orbit of $x$ gets trapped in the base $E_{0}$. That is, there exists $i_{0} \geq 0$ such that $S_{i_{0}}<\infty$ but $T_{i_{0}+1}(x)=\infty$. Then, for any $n_{0} \geq 1$, there 
exists $\epsilon_{1}>0$ such that if $x<y<x+\epsilon_{1}$ then $S_{i}(y)=S_{i}^{+}$and $T_{i}(y)=T_{i}^{+}$for every $i \leq i_{0}$, and $T_{i_{0}+1}(y) \geq n_{0}$. Clearly, the function

$$
\begin{gathered}
\tilde{\alpha}(y)=-\sum_{i<i_{0}} \frac{1}{\left(f^{S_{i}^{+}(x)}\right)^{\prime}(y)} w_{T_{i+1}^{+}-S_{i}^{+}}\left(f^{S_{i}^{+}}(y)\right)-\frac{1}{\left(f^{S_{i_{0}}}\right)^{\prime}(y)} w_{n_{0}-S_{i_{0}}^{+}}\left(f^{S_{i_{0}}^{+}}(y)\right) \\
-\sum_{i \leq i_{0}} \frac{1}{\left(f^{\left.T_{i}^{+}\right)^{\prime}(y)}\right.} w_{S_{i}^{+}-T_{i}^{+}}\left(f^{T_{i}}(y)\right)
\end{gathered}
$$

is continuous on $\left[x, x+\epsilon_{1}\right)$. Choose $\epsilon_{2}<\epsilon_{1}$ such that if $x \leq y<x+\epsilon_{2}$ then $|\tilde{\alpha}(y)-\tilde{\alpha}(x)|<\epsilon / 4$. Using again the uniformity of the estimates in the proof of Proposition 3.9 (in particular of the exponentially decaying term of the series (44) for $i=i_{0}$ ), it is easy to see that if $n_{0}$ is large enough then $\left|\alpha(y)-\alpha^{+}(x)\right|<\epsilon$ for $y \in\left[x, x+\epsilon_{2}\right)$.

The third case occurs when $\hat{f}^{i_{0}}(x, 0)=(c, 0)$ for some $i_{0} \geq 0$. That is, there exists $i_{0} \geq 1$ such that $T_{i_{0}}(x)<\infty$ but $S_{i_{0}}(x)=\infty$, and $\alpha(x)$ is just a finite sum. (If $x=c$ then $i_{0}=1$.) Then there exists $\epsilon_{2}>0$ such that if $x<y<x+\epsilon_{2}$ then $T_{i}(y)=T_{i}^{+}$and $S_{i-1}(y)=S_{i-1}^{+}$ for every $i \leq i_{0}$, while $S_{i_{0}}(y) \geq n_{0}$. To finish, define

$$
\tilde{\alpha}(y)=-\sum_{i<i_{0}} \frac{1}{\left(f^{S_{i}^{+}}\right)^{\prime}(y)} w_{T_{i+1}^{+}-S_{i}^{+}}\left(f^{S_{i}^{+}}(y)\right)-\sum_{i<i_{0}} \frac{1}{\left(f^{T_{i}^{+}}\right)^{\prime}(y)} w_{S_{i}^{+}-T_{i}^{+}}\left(f^{T_{i}^{+}}(y)\right)
$$

and adapt the arguments from the first two cases. This ends the proof of (56) and of Theorem 2.4 .

\subsection{Divergence of the formal power series (Proposition 2.5)}

In this final subsection, we show that the formal power series $\alpha_{\text {cand }}(x)$ diverges for many $x$.

Proof of Proposition 2.5. - It is enough to show that the set of points such that $\lim \sup _{i}\left|\frac{v\left(f^{i}(x)\right)}{\left(f^{i+1}\right)^{\prime}(x)}\right|>0$ has the desired property. Let $A$ be as in the statement of the proposition. We shall build a decreasing sequence of closed sets

$$
A \supset \mathcal{K}_{n} \supset \mathcal{K}_{n+1}
$$

where each connected component of $\mathcal{K}_{n}$ is a closed interval with positive length, and a sequence of functions

$$
\Gamma_{n+1}:\left\{C \mid C \text { connected comp. of } \mathcal{K}_{n}\right\} \rightarrow \mathscr{P}\left(\left\{1, \ldots, n+i_{0}\right\}\right),
$$

where $\mathscr{P}(\{1, \ldots, n\})$ stands for the family of all subsets of $\left\{1, \ldots, n+i_{0}\right\}$, with the following properties:

i. If $C$ is a connected component of $\mathcal{K}_{n}$ and $x \in C$, then $\left|\frac{v\left(f^{j}(x)\right)}{\left(f^{j+1}\right)^{\prime}(x)}\right| \geq 2$ for every $j \in \Gamma_{n}(C)$.

ii. If $C$ is a connected component of $\mathcal{K}_{n}$, then $f^{n}$ is a diffeomorphism on $C$.

iii. If $C_{n+1} \subset C_{n}$ are connected components of $\mathcal{K}_{n}$ and $\mathcal{K}_{n+1}$ respectively, then $\Gamma_{n}\left(C_{n}\right) \subset$ $\Gamma_{n+1}\left(C_{n+1}\right)$.

iv. If $C_{n}$ is a connected component of $\mathcal{K}_{n}$, then there exists $m>n$ such that $\mathcal{K}_{m}$ has at least two connected components contained in $C_{n}$.

v. If $x \in \cap_{n} C_{n}$, where $C_{n}$ is a connected component of $\mathcal{K}_{n}$, then $\{x\}=\cap_{n} C_{n}$ and $\lim _{n} \# \Gamma_{n}\left(C_{n}\right)=\infty$. 
Note that (iv) and (v) imply that $\cap_{n} \mathcal{K}_{n}$ is a Cantor set. If $x \in \cap_{n} C_{n}$ then

$$
\left|\frac{v\left(f^{j}(x)\right)}{\left(f^{j+1}\right)^{\prime}(x)}\right| \geq 2
$$

holds for every $j \in \cup_{n} \Gamma_{n}\left(C_{n}\right)$, and condition (v) implies that $\cup_{n} \Gamma_{n}\left(C_{n}\right)$ is an infinite set. Denote

$$
\theta(c)=\left\{x \in I \mid f^{i}(x)=c, \text { for some } i \geq 0\right\} .
$$

Let $\mathcal{K}_{0} \subset A$ be a closed interval $[a, b], a \neq b$, with $a, b \notin \Theta(c)$, and $\Gamma_{0}\left(\mathcal{K}_{0}\right)=\varnothing$. Suppose that we have defined $\mathcal{K}_{n}$. Let $C$ be a connected component of $\mathcal{K}_{n}$. If $c \notin f^{n}(C)$ then $C$ is the unique connected component of $\mathcal{K}_{n+1}$ which intersects $C$ and $\Gamma_{n+1}(C)=$ $\Gamma_{n}(C)$. Otherwise, let $x \in C$ be such that $f^{n}(x)=c$. Since $c$ is the critical point, we have $\left(f^{n+i_{0}+1}\right)^{\prime}(x)=0$. Moreover $v\left(f^{n+i_{0}}(x)\right)=v\left(f^{i_{0}}(x)\right) \neq 0$, so

$$
\lim _{y \rightarrow x}\left|\frac{v\left(f^{n+i_{0}}(y)\right)}{\left(f^{n+i_{0}+1}\right)^{\prime}(y)}\right|=\infty .
$$

Let $\epsilon>0$ be such that if $0<|y-x| \leq \epsilon$ then $y \in C$ and

$$
\left|\frac{v\left(f^{n+i_{0}}(y)\right)}{\left(f^{n+i_{0}+1}\right)^{\prime}(y)}\right| \geq 2 .
$$

Choose two closed disjoint intervals $J_{1}$ and $J_{2}$ with positive lengths, such that $J_{1} \cup J_{2} \subset(x-\epsilon, x+\epsilon) \backslash\{x\}$ and $\Theta(c) \cap \partial\left(J_{1} \cup J_{2}\right)=\varnothing$. Then $J_{1}$ and $J_{2}$ will be the unique connected component of $\mathcal{K}_{n+1}$ that intersects $C$ and $\Gamma_{n+1}\left(J_{1}\right)=\Gamma_{n+1}\left(J_{2}\right)=\Gamma_{n}(C) \cup\left\{n+i_{0}\right\}$. Note that in this case $\Gamma_{n+1}\left(J_{1}\right)=\Gamma_{n+1}\left(J_{2}\right) \supseteqq \Gamma_{n}(C)$.

Properties (i)-(iii) follow from the definition of $\mathcal{K}_{n}$. To show (iv) and (v), consider $C_{\infty}=\cap_{n} C_{n}$, where the $C_{n}$ are the connected components of $\mathcal{K}_{n}$ containing $x$. The set $C_{\infty}$ is either a closed interval of positive length or $\{x\}$. In the first case, in particular we have that $f^{n}$ is a diffeomorphism on $C_{\infty}$, for every $n$. This is not possible, since $f$ has neither wandering intervals nor periodic attractors. Furthermore, note that if $\lim _{n} \# \Gamma_{n}\left(C_{n}\right)<\infty$, then there exists $n_{0}$ such that $\Gamma_{n}\left(C_{n}\right)=\Gamma_{n_{0}}\left(C_{n_{0}}\right)$ for every $n \geq n_{0}$, so by the construction of $\mathcal{K}_{i}$ this occurs only if $f^{i}$ is a diffeomorphism on $C_{n_{0}}$ for every $i$, which is not possible, as we saw above. The proof of (iv) is similar. If (iv) does not hold for certain $C_{n_{0}}$, then by the construction of $\mathcal{K}_{i}$ we have that $f^{i}$ is a diffeomorphism on $C_{n_{0}}$ for every $i$, which contradicts the non-existence of wandering intervals and periodic attractors.

\section{Transfer operators $\widehat{\mathscr{L}}$ and their spectra}

In this section, we study a transfer operator associated to a Collet-Eckmann $S$-unimodal map $f$ satisfying the Benedicks-Carleson condition. More precisely, in Subsection 4.1, we introduce a Banach space $\mathscr{B}=\mathscr{B}^{1}$ of smooth $\left(W_{1}^{1}\right)$ functions (see Definition 4.3) on the tower $\hat{I}$ defined in the previous section, maps $\Pi: \mathscr{B} \rightarrow L^{1}(I)$ (see (72)), as well as a transfer operator $\widehat{\mathscr{L}}$ acting on $\mathscr{B}$ (Definition 4.8). We shall prove that $\widehat{\mathscr{L}}$ has essential spectral radius strictly smaller than 1 (Proposition 4.10), and then (Proposition 4.11) that 1 is a simple eigenvalue, and that the fixed point $\hat{\phi}$ of $\widehat{\mathscr{L}}$ is such that $\Pi(\hat{\phi})$ is the invariant density of $f$. In Subsection 4.2 , we present results of truncated versions of $\widehat{\mathcal{L}}$, acting on finite parts of the tower. 
The methods in this section are inspired from [15], but we would like to point out here that nontrivial modifications were needed in view of proving Theorem 2.13. (See Remark 4.4 below for the comparison with [51].) Also, the transfer operator we use here is slightly different from the one in [15]: First, and this is the most original ingredient, we introduce a smooth cutoff function $\xi_{k}$ at each level on which there exist points which "fall" to the ground level; second, we do not compose with the dynamics until we fall (this strategy was used by L.S. Young in [61], [62]). Finally, our Banach spaces $\mathscr{B}$ are not exactly the same as the space $\widehat{\mathcal{B}}$ used in [15]: The functions in $\mathscr{B}$ are smooth and locally supported at each level (this is possible in view of the other two changes). These are the main new ideas in the present section, and they allow to circumscribe the effect of the discontinuities and square root singularities (the spikes) to the maps $\Pi$ and $\Pi_{t}$ (see Step 3 in the proof of Theorem 2.13). See also the comments after Definition 4.8.

\subsection{Spectral gap for a transfer operator $\widehat{\mathscr{L}}$ associated to the tower map}

Let $f$ be a $\left(\lambda_{c}, H_{0}\right)$-Collet-Eckmann $S$-unimodal map, with a non preperiodic critical point (the preperiodic case is easier and left to the reader) satisfying the Benedicks-Carleson condition (3) for $\mathscr{G}=4$. (We shall need to take larger values for $\mathscr{G}$ below, see also (109).) We consider the tower map $\hat{f}: \hat{I} \rightarrow \hat{I}$ from Section 3.1, for some small enough fixed $\delta$. We shall not require the fact that the Lyapunov exponents of $\pm \delta$, or of the endpoints $a_{k}$ and $b_{k}$ of the tower levels $B_{k}$, are positive, and we remove ${ }^{(16)}$ this assumption. (The positivity of the exponents was useful only when proving that $\alpha_{t}$ is continuous, and one can use different towers for $f_{t}$ when studying $\alpha$ and when considering the transfer operator.) In particular, recalling $\beta_{1}$ from (27), we may take for all $k \geq H_{0}$

$$
B_{k}=\left[c_{k}-e^{-\beta_{1} k}, c_{k}+e^{-\beta_{1} k}\right] .
$$

The following refinement of the estimates in Subsection 3.2 will play an important part in our argument (see Proposition 4.10, and - in view of (72)—Proposition 4.11, see also Step 2 in the proof of Theorem 2.13):

Lemma 4.1. - Let $f$ be an S-unimodal $\left(\lambda_{c}, H_{0}\right)$-Collet-Eckmann map satisfying the Benedicks-Carleson condition (3) for $\mathscr{G}=4$, and with a non-preperiodic critical point. Then there exists $C$ so that for any $k \geq 1$, we have

$$
\frac{1}{\left|\left(f^{k}\right)^{\prime}\left(f_{ \pm}^{-k}(x)\right)\right|} \leq C \frac{1}{\left|\left(f^{k-1}\right)^{\prime}\left(c_{1}\right)\right|^{1 / 2} \sqrt{\left|x-c_{k}\right|}}, \quad \forall x \in \pi\left(E_{k} \cap \hat{f}^{k}\left(E_{0}\right)\right) .
$$

In addition, recalling the intervals $I_{k}$ defined in (32), we have for any $k \geq H(\delta)$

$$
\sup _{x \in f^{k}\left(I_{k}\right)}\left|\partial_{x} \frac{1}{\left|\left(f^{k}\right)^{\prime}\left(f_{ \pm}^{-k}(x)\right)\right|}\right| \leq C \frac{e^{3 \gamma k}}{\left|\left(f^{k-1}\right)^{\prime}\left(c_{1}\right)\right|^{1 / 2}},
$$

and

$$
\sup _{x \in f^{k}\left(I_{k}\right)}\left|\partial_{x}^{2} \frac{1}{\left|\left(f^{k}\right)^{\prime}\left(f_{ \pm}^{-k}(x)\right)\right|}\right| \leq C C \frac{e^{5 \gamma k}}{\left|\left(f^{k-1}\right)^{\prime}\left(c_{1}\right)\right|^{1 / 2}}
$$

(16) This additional freedom will also be used in Subsection 5.2.

$4^{\text {e }}$ SÉRIE - TOME $45-2012$ - No 6 
Proof. - We consider the case $\varsigma=+$, the other case is identical. Let us first show (58). Putting $z=f_{+}^{-(k-1)}(x)$, we decompose

$$
\left|\left(f^{k}\right)^{\prime}\left(f_{+}^{-k}(x)\right)\right|=\left|\left(f^{k-1}\right)^{\prime}(z)\right|\left|f^{\prime}\left(f_{+}^{-k}(x)\right)\right| .
$$

By Lemma 3.3, the first factor can be estimated by ${ }^{(17)}$

$$
\left|\left(f^{k-1}\right)^{\prime}(z)\right| \geq C^{-1}\left|\left(f^{k-1}\right)^{\prime}\left(c_{1}\right)\right| .
$$

For the second factor, we have

$$
\left|f^{\prime}\left(f_{+}^{-k}(x)\right)\right| \geq C^{-1}\left|f_{+}^{-k}(x)-c\right| .
$$

Put $w=f\left(f_{+}^{-k}(x)\right)$. Then, Lemma 3.3 and the mean value theorem imply

$$
\left|w-c_{1}\right| \geq \frac{\left|x-c_{k}\right|}{C\left|\left(f^{k-1}\right)^{\prime}\left(c_{1}\right)\right|} .
$$

Next, noting that $w \in \pi\left(E_{1} \cap \hat{f}\left(E_{0}\right)\right)$, we have

$$
\left|f_{+}^{-k}(x)\right|=\left|f_{+}^{-1}(w)\right| \geq C^{-1} \sqrt{\left|w-c_{1}\right|} .
$$

(Just use that $f(y)=c_{1}+f^{\prime \prime}(c) y^{2}+f^{\prime \prime \prime}(\tilde{y}) y^{3}$ for some $|\tilde{y}| \leq \delta$, if $|y| \leq \delta$.) The three previous inequalities give

$$
\left|f^{\prime}\left(f_{+}^{-k}(x)\right)\right| \geq C^{-1} \frac{\sqrt{\left|x-c_{k}\right|}}{\left|\left(f^{k-1}\right)^{\prime}\left(c_{1}\right)\right|^{1 / 2}} .
$$

Putting together (61) and (62), we get (58).

To prove (59), we first note that there is $C \geq 1$ so that

$$
\left|f^{\prime}\left(f^{j}(y)\right)\right| \geq C^{-1} e^{-\gamma j}, \forall y \in I_{k}, \forall 1 \leq j \leq k .
$$

Indeed, $\left|f^{j}(y)-c_{j}\right| \leq e^{-\beta_{1} j}$ and $\left|c_{j}-c\right| \geq e^{-\gamma k}$ for $j \geq H_{0}$, with $\beta_{1} \geq 3 \gamma / 2$, and we assumed that $f$ is $C^{2}$ with $f^{\prime}(c)=0$ and $f^{\prime \prime}(c) \neq 0$. Next, Lemma 3.3 and Lemma 3.7 give $C>0$ so that

$$
\sup _{y \in I_{k}} \frac{1}{\left|\left(f^{k-j}\right)^{\prime}\left(f^{j}(y)\right)\right|} \leq C \sum_{\ell=1}^{\infty} \frac{1}{\left|\left(f^{\ell}\right)^{\prime}\left(f^{j-1}\left(c_{1}\right)\right)\right|} \leq C e^{\gamma j}, \forall 1 \leq j \leq k-1 .
$$

Then, (36) from Lemma 3.4 gives

$$
\sup _{y \in I_{k}} \frac{1}{\left|\left(f^{m}\right)^{\prime}(y)\right|} \leq C_{2}^{-1} e^{\gamma k} \frac{1}{\left|\left(f^{m-1}\right)^{\prime}\left(c_{1}\right)\right|^{1 / 2}}, \quad \forall 1 \leq m \leq k
$$

Applying (63) and (64), we get $C>0$ so that

$$
\begin{aligned}
\sup _{y \in I_{k}} \partial_{y} \frac{1}{\left|\left(f^{k}\right)^{\prime}(y)\right|} & \leq \sup _{y \in I_{k}} \sum_{j=0}^{k-1} \frac{\left|f^{\prime \prime}\left(f^{j}(y)\right)\right|}{\left|\left(f^{k}\right)^{\prime}(y)\right|} \frac{\left|\left(f^{j}\right)^{\prime}(y)\right|}{\left|f^{\prime}\left(f^{j}(y)\right)\right|} \\
& =\sup _{y \in I_{k}} \sum_{j=0}^{k-1} \frac{\left|f^{\prime \prime}\left(f^{j}(y)\right)\right|}{\left|\left(f^{k-j}\right)^{\prime}\left(f^{j}(y)\right)\right|} \frac{1}{\left|f^{\prime}\left(f^{j}(y)\right)\right|} \leq C e^{2 \gamma k} .
\end{aligned}
$$

Finally,

$$
\partial_{x} \frac{1}{\left|\left(f^{k}\right)^{\prime}\left(f_{+}^{-k}(x)\right)\right|}=\frac{1}{\left|\left(f^{k}\right)^{\prime}\left(f_{+}^{-k}(x)\right)\right|} \cdot \partial_{y} \frac{1}{\left|\left(f^{k}\right)^{\prime}(y)\right|}
$$

$\overline{\text { (17) The constant } C}$ depends on $H_{0}$, by Lemma 5.8 it will be uniform within our families $f_{t}$. 
The first factor in (67) is bounded by (65) for $m=k$, the second by (66), so that we have proved (59).

To prove (60), we start from the decomposition (67), and we deduce from (66) that for any $x \in f^{k}\left(I_{k}\right)$, setting $y=f_{+}^{-k}(x)$,

$$
\begin{aligned}
\partial_{x}^{2} \frac{1}{\left|\left(f^{k}\right)^{\prime}\left(f_{+}^{-k}(x)\right)\right|} \leq & C e^{2 \gamma k} \partial_{x} \frac{1}{\left|\left(f^{k}\right)^{\prime}\left(f_{+}^{-k}(x)\right)\right|} \\
& +\frac{1}{\left|\left(f^{k}\right)^{\prime}\left(f_{+}^{-k}(x)\right)\right|} \cdot \sum_{j=0}^{k-1} \partial_{x}\left(\frac{\left|f^{\prime \prime}\left(f^{j}(y)\right)\right|}{\left|\left(f^{k-j}\right)^{\prime}\left(f^{j}(y)\right)\right|\left|f^{\prime}\left(f^{j}(y)\right)\right|}\right) .
\end{aligned}
$$

By (59), the first term in the right-hand side is bounded by $C e^{5 \gamma k}\left|\left(f^{k-1}\right)^{\prime}\left(c_{1}\right)\right|^{-1 / 2}$. For the second term, we have, for $0 \leq j \leq k-1$,

$$
\partial_{x} \frac{\left|f^{\prime \prime}\left(f^{j}(y)\right)\right|}{\left|\left(f^{k-j}\right)^{\prime}\left(f^{j}(y)\right)\right|\left|f^{\prime}\left(f^{j}(y)\right)\right|} \leq \frac{1}{\left|\left(f^{k}\right)^{\prime}\left(f_{+}^{-k}(x)\right)\right|} \partial_{y} \frac{\left|f^{\prime \prime}\left(f^{j}(y)\right)\right|}{\left|\left(f^{k-j}\right)^{\prime}\left(f^{j}(y)\right)\right|\left|f^{\prime}\left(f^{j}(y)\right)\right|} .
$$

Since $f$ is $C^{3}$, the Leibniz formula gives for $0 \leq j \leq k-1$,

$$
\begin{aligned}
& \frac{1}{\left|\left(f^{k}\right)^{\prime}\left(f_{+}^{-k}(x)\right)\right|} \partial_{y} \frac{\left|f^{\prime \prime}\left(f^{j}(y)\right)\right|}{\left|\left(f^{k-j}\right)^{\prime}\left(f^{j}(y)\right)\right|\left|f^{\prime}\left(f^{j}(y)\right)\right|} \\
& \leq \frac{1}{\left|\left(f^{k}\right)^{\prime}\left(f_{+}^{-k}(x)\right)\right|} \frac{1}{\left|\left(f^{k-j}\right)^{\prime}\left(f^{j}(y)\right)\right|\left|f^{\prime}\left(f^{j}(y)\right)\right|} \cdot\left[\left|f^{\prime \prime \prime}\left(f^{j}(y)\right)\right|\left|\left(f^{j}\right)^{\prime}(y)\right|\right. \\
& \left.\quad+\left|f^{\prime \prime}\left(f^{j}(y)\right)\right|\left[\frac{\left|f^{\prime \prime}\left(f^{j}(y)\right)\right|\left|\left(f^{j}\right)^{\prime}(y)\right|}{\left|f^{\prime}\left(f^{j}(y)\right)\right|}+\sum_{\ell=j}^{k-1} \frac{\left|f^{\prime \prime}\left(f^{\ell}(y)\right)\right|\left|\left(f^{\ell}\right)^{\prime}(y)\right|}{\left|f^{\prime}\left(f^{\ell}(y)\right)\right|}\right]\right] \\
& \leq \frac{C}{\left|\left(f^{k-j}\right)^{\prime}\left(f^{j}(y)\right)\right|} \cdot\left[\frac { 1 } { | ( f ^ { k - j } ) ^ { \prime } ( f ^ { j } ( y ) ) | } \left(\frac{1}{\left|f^{\prime}\left(f^{j}(y)\right)\right|}+\frac{1}{\left.\left|f^{\prime}\left(f^{j}(y)\right)\right|^{2}\right)}\right.\right. \\
& \left.\quad+\frac{1}{\left|\left(f^{k}\right)^{\prime}(y)\right|} \sum_{\ell=j}^{k-1} \frac{\left|\left(f^{\ell}\right)^{\prime}(y)\right|}{\left|f^{\prime}\left(f^{\ell}(y)\right)\right|}\right] .
\end{aligned}
$$

If $j \geq 1$, we may apply (63). Then, (69), together with (65) for $m=k-\ell$ and (64) imply that

$$
\begin{gathered}
\frac{1}{\left|\left(f^{k}\right)^{\prime}\left(f_{+}^{-k}(x)\right)\right|} \sum_{j=1}^{k-1} \partial_{y} \frac{\left|f^{\prime \prime}\left(f^{j}(y)\right)\right|}{\left|\left(f^{k-j}\right)^{\prime}\left(f^{j}(y)\right)\right|\left|f^{\prime}\left(f^{j}(y)\right)\right|} \\
\leq C e^{\gamma k} \cdot\left[e^{2 \gamma k}+e^{3 \gamma k}+e^{2 \gamma k}\right] .
\end{gathered}
$$

If $j=0$, then (69) together with (37) and (65) for $m=k$ imply (distinguish between $\ell=0$ and $\ell \geq 1$ )

$$
\begin{aligned}
& \frac{1}{\left|\left(f^{k}\right)^{\prime}\left(f_{+}^{-k}(x)\right)\right|} \partial_{y} \frac{\left|f^{\prime \prime}(y)\right|}{\left|\left(f^{k}\right)^{\prime}(y)\right|\left|f^{\prime}(y)\right|} \\
& \quad \leq C \frac{e^{3 \gamma k}}{\left|\left(f^{k}\right)^{\prime}\left(c_{1}\right)\right|^{1 / 2}}\left(e^{\gamma k}+e^{2 \gamma k}\left|\left(f^{k}\right)^{\prime}\left(c_{1}\right)\right|^{1 / 2}+e^{2 \gamma k}\right) .
\end{aligned}
$$

Putting the two above inequalities together with (68) and (65) for $m=k$, we get (60).

In view of the definition of our Banach space $\mathcal{B}$, we need further preparations. First, we assume from now on that $f$ satisfies the Benedicks-Carleson condition (3) for $\mathscr{G}=8$. 
REMARK 4.2. - In [15] we needed to assume that $f$ was $C^{4}$ or symmetric (see the comments before [15, Lemma 5]) because of the more complicated form of the cocycle used in the transfer operator there.

In view of (3) for $\mathscr{G}=8$ we may choose $\lambda$ so that

$$
1<\lambda<e^{\gamma} \text {, and } e^{4 \gamma} \lambda<\sqrt{\lambda}_{c} .
$$

It does not seem possible to work on spaces of sequences of $C^{1}$ functions $\psi_{k}$ (when summing over critical inverse branches in the proof of Proposition 4.10, bounded distortion would allow us to replace $\left|\left(f^{k}\right)^{\prime}(x)\right|^{-1}$ by the square root of the length of the corresponding monotonicity interval, instead of the length itself), and it will be convenient to work with Sobolev spaces: For integer $r \geq 0$, we recall that the generalized Sobolev norm of a function $\psi: I \rightarrow \mathbb{C}$, compactly supported in the interior of $I$, is

$$
\|\psi\|_{W_{1}^{r}}=\left\|\partial_{x}^{r} \varphi(x)\right\|_{L^{1}} .
$$

Compactly supported $C^{\infty}$ functions are dense in $W_{1}^{r}$ for $r \geq 1$. The Sobolev embedding in dimension one gives $\|\cdot\|_{C^{0}} \leq C\|\cdot\|_{W_{1}^{1}}$ and $\|\cdot\|_{C^{1}} \leq C\|\cdot\|_{W_{1}^{2}}$.

Definition 4.3 (The main Banach space $\mathscr{B}=\mathscr{B}^{W_{1}^{1}}$ ). - Let $\mathscr{B}=\mathscr{B}^{W_{1}^{1}}$ be the space of sequences $\hat{\psi}=\left(\psi_{k}: I \rightarrow \mathbb{C}, k \in \mathbb{Z}_{+}\right)$, so that each $\psi_{k}$ is in $W_{1}^{1}$ and, in addition,

$$
\begin{aligned}
& \operatorname{supp}\left(\psi_{0}\right) \subset(-1,1), \quad \operatorname{supp}\left(\psi_{k}\right) \subset[-\delta, \delta], \forall 1 \leq k \leq \max \left(2, H_{0}\right), \\
& \operatorname{supp}\left(\psi_{k}\right) \subset \cap_{H_{0} \leq j \leq k}\left(f_{+}^{-j}\left(B_{j}\right) \cup f_{-}^{-j}\left(B_{j}\right)\right), \forall k>\max \left(2, H_{0}\right),
\end{aligned}
$$

endowed with the norm

$$
\|\hat{\psi}\|_{\mathcal{B}^{W_{1}^{1}}}=\sum_{k \geq 0}\left\|\psi_{k}\right\|_{W_{1}^{1}}
$$

We sometimes write $\hat{\psi}(x, k)$ instead of $\psi_{k}(x)$.

REMARK 4.4. - In contradistinction to the piecewise expanding case treated in [10], or to the Misiurewicz and analytic case studied in [51], the postcritical data is not given here by a finite set of complex numbers for each $c_{k}$ with $k \geq 1$ : We need a full "germ" $\psi_{k}$, which is supported in a neighborhood of $c$. Since we shall later consider $\left(\psi_{k} \circ f_{ \pm}^{-k}\right) \chi_{k}$ (recall $\chi_{k}$ from Proposition 2.7, and see (72)), we can view $\psi_{k}$ as the contribution in a one-sided neighborhood of $c_{k}$.

Definition 4.5 (The projection $\Pi)$. - Define $\Pi(\hat{\psi})$ for $\hat{\psi} \in \mathcal{B}$ by

$$
\Pi(\hat{\psi})(x)=\sum_{k \geq 0, \varsigma \in\{+,-\}} \frac{\lambda^{k}}{\left|\left(f^{k}\right)^{\prime}\left(f_{\varsigma}^{-k}(x)\right)\right|} \psi_{k}\left(f_{\varsigma}^{-k}(x)\right) \chi_{k}(x) .
$$

(We set $\chi_{0} \equiv 1$. When the meaning is clear, we sometimes omit the factor $\chi_{k}$ in the formula.) 
By (58) in Lemma 4.1 and our construction ${ }^{(18)}$, setting $\left[c_{k}, d_{k}\right]=\pi\left(E_{k} \cap \hat{f}^{k}\left(E_{0}\right)\right)$,

$$
\begin{aligned}
\int_{\left[c_{k}, d_{k}\right]} \frac{\lambda^{k}}{\left|\left(f^{k}\right)^{\prime}\left(f_{+}^{-k}(x)\right)\right|}\left|\psi_{k}\left(f_{+}^{-k}(x)\right)\right| d x & \leq C \lambda^{k} \lambda_{c}^{-k / 2} \sqrt{\left|d_{k}-c_{k}\right|} \sup \left|\psi_{k}\right| \\
& \leq C\left(\lambda \lambda_{c}^{-1 / 2} e^{-3 \gamma / 4}\right)^{k} \sup \left|\psi_{k}\right| .
\end{aligned}
$$

Since $\lambda<\sqrt{\lambda_{c}}$, the above bound, and its analogue for the branch $f_{-}^{-k}$, imply that $\Pi(\hat{\psi}) \in L^{1}(I)$ for $\hat{\psi} \in \mathcal{B}$.

We shall need a weak norm, in order to write Lasota-Yorke inequalities. Set

$$
w(x, k)=\lambda^{k}, \quad x \in I, k \geq 0,
$$

and define $\nu$ to be the nonnegative measure on $\cup_{k \geq 0}\{k\} \times I$ whose density with respect to Lebesgue is $w(x, k)$.

Definition 4.6 (Space $\left.\mathscr{B}^{L^{1}(\nu)}\right)$. - Let $\mathscr{B}^{L^{1}}=\mathscr{B}^{L^{1}(\nu)}$ be the space of sequences $\hat{\psi}$ of functions $\psi_{k}$, with the norm

$$
\|\hat{\psi}\|_{\mathcal{B}^{L^{1}}}=\sum_{k \geq 0} \lambda^{k}\left\|\psi_{k}\right\|_{L^{1}(I)}=\|\hat{\psi}\|_{L^{1}(\nu)}
$$

In order to define the transfer operator $\widehat{\mathscr{L}}$, we next introduce smooth cutoff functions $\xi_{k}$. Recall the constants $3 \gamma / 2<\beta_{1}<\beta_{2}<2 \gamma$ from (27) and (28).

Definition 4.7 (The cutoff functions $\xi_{k}$ ). - For each $k \geq 0$, let $\xi_{k}: I \rightarrow[0,1]$ be a $C^{\infty}$ function satisfying the following conditions: $\xi_{k} \equiv 1$ for those levels $k$ from which no point falls to level 0 and otherwise

$$
\begin{aligned}
& \operatorname{supp}\left(\xi_{0}\right)=[-\delta, \delta],\left.\quad \xi_{0}\right|_{\left[-\frac{\delta}{2}, \frac{\delta}{2}\right]} \equiv 1, \\
& k \geq H(\delta):\left\{\begin{array}{l}
\operatorname{supp}\left(\xi_{k}\right)=f_{+}^{-(k+1)}\left(B_{k+1}\right) \cup f_{-}^{-(k+1)}\left(B_{k+1}\right), \\
\left.\xi_{k}\right|_{f_{+}^{-(k+1)}\left[c_{k+1}-e^{-\beta_{2}(k+1)}, c_{k+1}+e^{-\beta_{2}(k+1)}\right]} \equiv 1, \\
\xi_{k} \text { is unimodal, }
\end{array}\right.
\end{aligned}
$$

$\left|\partial_{x}^{j}\left(\xi_{0} \circ f_{ \pm}^{-1}(x)\right)\right| \leq c(\delta)^{-j}$ for $j=1,2,3$, and, finally, that $\beta_{1}$ is close enough to $3 \gamma / 2$ and $\beta_{2}$ is close enough to $2 \gamma$ so that for some $C>0$

$$
\sup \left|\partial_{x}^{j}\left(\xi_{k} \circ f_{ \pm}^{-(k+1)}(x)\right)\right| \leq C e^{2 j \gamma k}, j=1,2,3 .
$$

Note that $\xi_{k}(y)>0$ if and only if $\hat{f}\left(f^{k}(y), k\right) \in B_{k+1} \times(k+1)$, and $\xi_{k}(y)=1$ implies that $\pi \hat{f}\left(f^{k}(y), k\right) \in\left[c_{k+1}-e^{-\beta_{2}(k+1)}, c_{k+1}+e^{-\beta_{2}(k+1)}\right]$. The low levels $(k \leq H(\delta))$ will be taken care of by the condition $\operatorname{supp}\left(\psi_{k}\right) \subset[-\delta, \delta]$.

Definition 4.8 (Transfer operator). - The transfer operator $\widehat{\mathscr{L}}$ is defined on $\mathscr{B}$ by

$$
(\widehat{\mathscr{L}} \widehat{\psi})(x, k)= \begin{cases}\frac{\xi_{k-1}(x)}{\lambda} \cdot \hat{\psi}(x, k-1) & k \geq 1 \\ \sum_{j \geq 0, \varsigma \in\{+,-\}} \frac{\lambda^{j}\left(1-\xi_{j}\left(f_{\varsigma}^{-(j+1)}(x)\right)\right)}{\left|\left(f^{j+1}\right)^{\prime}\left(f_{\varsigma}^{-(j+1)}(x)\right)\right|} \cdot \hat{\psi}\left(f_{\varsigma}^{-(j+1)}(x), j\right) & k=0\end{cases}
$$

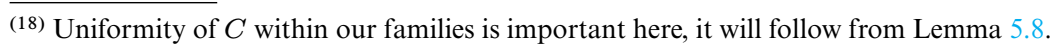

$4^{\text {e }}$ SÉRIE - TOME $45-2012$ - No 6 
Some $j$-terms in the sum for $(\widehat{\mathcal{L}} \widehat{\psi})(x, 0)$ vanish, in particular, for all $1 \leq j<H_{0}$ because of our choice of small $\delta$ giving $H(\delta) \geq H_{0}$.

As already mentioned in the beginning of this section, there are two differences between the present definition and the one used in [15]. First, $\widehat{\mathcal{L}}$ does not act via the dynamics when climbing the tower, only when falling. Secondly, if $0<\xi_{j}(y)<1$, then $y$ will contribute to both $(\widehat{\mathscr{L}} \widehat{\psi})(y, j+1)$ and $(\widehat{\mathscr{L}} \widehat{\psi})\left(f^{j+1}(y), 0\right)$. In other words, the transfer operator just defined is associated to a multivalued (probabilistic-type) tower dynamics. For this multivalued dynamics, some points may fall from the tower a little earlier than they would for $\hat{f}$. However, the conditions on the functions $\xi_{k}$ s guarantee that they do not fall too early. More precisely, if we define "fuzzy" analogues of the intervals $I_{k}$ from (32) as follows

$$
\widetilde{I}_{k}:=\left\{x \in I \mid \xi_{k}(x)<1, \xi_{j}(x)>0, \forall 0 \leq j<k\right\},
$$

then we can replace $I_{k}$ by $\widetilde{I}_{k}$ in the previous estimates, in particular in Lemma 4.1. Indeed, just observe that if a point "falls" according to our fuzzy dynamics, it would have fallen for some choice of intervals $\widetilde{B}_{k}$ so that

$$
\left[c_{k}-e^{-\beta_{2} k}, c_{k}+e^{-\beta_{2} k}\right] \subset \widetilde{B}_{k} \subset B_{k} .
$$

Remark 4.9. - The intervals $\widetilde{I}_{k}$ do not have pairwise disjoint interiors. However, for each $k$, the cardinality of those $\widetilde{I}_{j}$ whose interiors intersect the interior of $\widetilde{I}_{k}$ is bounded by $k$ for $k \geq N(f)$, where $N(f)$ depends only on $\sigma$ from Lemma 3.1 and on $\gamma$ (the smaller $\gamma$, the shorter this waiting time). We may assume by taking smaller $\delta$ that $N(f) \leq H(\delta)$. Indeed, if a point falls $x$ from level $k$ for the first time with the fuzzy dynamics, it will fall for the last time at level $2 k$, because Lemma 3.1 for $\delta=e^{-4 \gamma k}$ together with the BenedicksCarleson assumption give $\left|\left(f^{k}\right)^{\prime}(x)\right| \geq c e^{-4 \gamma k} \sigma^{k}$, and $c e^{-4 \gamma k} \sigma^{k} e^{-2 \gamma k}>e^{-\frac{3}{2} 2 k}$. (The present remark will be used to get the Lasota-Yorke estimate at the heart of Proposition 4.10, see Appendix B.)

These two modifications allow us to work with Sobolev spaces $W_{1}^{r}$ (as opposed to the BV functions in [15], where the jump singularities corresponding to the edges of the levels are an artifact of the construction), and will simplify our spectral perturbation argument in Section 6.

Before we continue, let us note that if we introduce the ordinary (Perron-Frobenius) transfer operator

$$
\mathscr{L}: L^{1}(I) \rightarrow L^{1}(I), \quad \mathscr{L} \varphi(x)=\sum_{f(y)=x} \frac{\varphi(y)}{\left|f^{\prime}(y)\right|}
$$

then one easily shows that

$$
\mathscr{L}(\Pi(\hat{\psi}))=\Pi(\widehat{\mathscr{L}}(\hat{\psi}))
$$


Indeed, on the one hand, we have (recall $\left.\chi_{0} \equiv 1\right)$

$$
\begin{aligned}
& {[\Pi \widehat{\mathscr{L}}(\hat{\psi})](x)=[\widehat{\mathscr{L}}(\hat{\psi})](x, 0) \chi_{0}(x)} \\
& \quad+\sum_{k \geq 1, \varsigma \in\{+,-\}} \frac{\lambda^{k}}{\left|\left(f^{k}\right)^{\prime}\left(f_{\varsigma}^{-k}(x)\right)\right|}[\widehat{\mathscr{L}}(\hat{\psi})]\left(f_{\varsigma}^{-k}(x), k\right) \chi_{k}(x) \\
& =\sum_{j \geq 0, \varsigma \in\{+,-\}} \frac{\lambda^{j}}{\left|\left(f^{(j+1)}\right)^{\prime} f_{\varsigma}^{-(j+1)}(x)\right|} \psi_{j}\left(f_{\varsigma}^{-(j+1)}(x)\right)\left(1-\xi_{j}\right)\left(f_{\varsigma}^{-(j+1)}(x)\right) \chi_{1}(x) \\
& \quad+\sum_{k \geq 1, \varsigma \in\{+,-\}} \frac{\lambda^{k-1}}{\left|\left(f^{k}\right)^{\prime}\left(f_{\varsigma}^{-k}(x)\right)\right|} \psi_{k-1}\left(f_{\varsigma}^{-k}(x)\right) \xi_{k-1}\left(f_{\varsigma}^{-k}(x)\right) \chi_{k}(x) \\
& =\sum_{j \geq 0, \varsigma \in\{+,-\}} \frac{\lambda^{j}}{\left|\left(f^{(j+1)}\right)^{\prime} f_{\varsigma}^{-(j+1)}(x)\right|} \psi_{j}\left(f_{\varsigma}^{-(j+1)}(x)\right)\left(1-\xi_{j}\right)\left(f_{\varsigma}^{-(j+1)}(x)\right) \chi_{1}(x) \\
& \quad+\sum_{j \geq 0, \varsigma \in\{+,-\}} \frac{\lambda^{j}}{\left|\left(f^{(j+1)}\right)^{\prime}\left(f_{\varsigma}^{-(j+1)}(x)\right)\right|} \psi_{j}\left(f_{\varsigma}^{-(j+1)}(x)\right) \xi_{j}\left(f_{\varsigma}^{-(j+1)}(x)\right) \chi_{j+1}(x) .
\end{aligned}
$$

On the other hand

$$
\begin{aligned}
(\mathscr{L} \Pi \hat{\psi}) & \left.(x)=\sum_{f(y)=x} \frac{\chi_{1}(x)}{\left|f^{\prime}(y)\right|} \sum_{\ell \geq 0, \varsigma \in\{+,-\}} \frac{\lambda^{\ell}}{\left|\left(f^{\ell}\right)^{\prime}\left(f_{\varsigma}^{-\ell}(y)\right)\right|} \psi_{\ell}\left(f_{\varsigma}^{-\ell}(y)\right) \chi_{\ell}(y)\right) \\
= & \sum_{f(y)=x} \frac{\chi_{1}(x)}{\left|f^{\prime}(y)\right|}\left(\sum_{\ell \geq 0, \varsigma \in\{+,-\}} \frac{\lambda^{\ell}}{\left|\left(f^{\ell}\right)^{\prime}\left(f_{\varsigma}^{-\ell}(y)\right)\right|} \psi_{\ell}\left(f_{\varsigma}^{-\ell}(y)\right)\left(1-\xi_{\ell}\left(f_{\varsigma}^{-\ell}(y)\right) \chi_{\ell}(y)\right)\right. \\
& +\sum_{f(y)=x} \frac{\chi_{1}(x)}{\left|f^{\prime}(y)\right|}\left(\sum_{\ell \geq 0, \varsigma \in\{+,-\}} \frac{\lambda^{\ell}}{\left|\left(f^{\ell}\right)^{\prime}\left(f_{\varsigma}^{-\ell}(y)\right)\right|} \psi_{\ell}\left(f_{\varsigma}^{-\ell}(y)\right) \xi_{\ell}\left(f_{\varsigma}^{-\ell}(y)\right) \chi_{\ell}(y)\right) \\
= & \sum_{f(y)=x}\left(\sum_{j \geq 0, \varsigma \in\{+,-\}} \frac{\lambda^{j}}{\left|\left(f^{(j+1)}\right)^{\prime}\left(f_{\varsigma}^{-j}(y)\right)\right|} \psi_{j}\left(f_{\varsigma}^{-j}(y)\right)\left(1-\xi_{j}\left(f_{\varsigma}^{-j}(y)\right) \chi_{1}(x) \chi_{j}(y)\right)\right. \\
& +\sum_{f(y)=x}\left(\sum_{j \geq 0, \varsigma \in\{+,-\}} \frac{\lambda^{j}}{\left|\left(f^{(j+1)}\right)^{\prime}\left(f_{\varsigma}^{-j}(y)\right)\right|} \psi_{j}\left(f_{\varsigma}^{-j}(y)\right) \xi_{j}\left(f_{\varsigma}^{-j}(y)\right) \chi_{1}(x) \chi_{j}(y)\right) .
\end{aligned}
$$

Now, our definitions ensure that

$$
\xi_{j}\left(f_{\varsigma}^{-j}(y)\right) \neq 0 \Longrightarrow \chi_{j}(y) \chi_{1}(f(y))=\chi_{j+1}(f(y)),
$$

and

$$
\psi_{j}\left(f_{\varsigma}^{-j}(y)\right)\left(1-\xi_{j}\left(f_{\varsigma}^{-j}(y)\right)\right) \neq 0 \Longrightarrow \chi_{j}(y)=1 .
$$

Finally, the sums over inverse branches coincide: Distinguish between zero level - where it is obvious - and other levels - where the last sum $(f(y)=x)$ over two inverse branches has at most one nonzero contribution by the support properties of the $\psi_{k}$ and $\xi_{k}$. This proves (78). In particular, if $\hat{\mathscr{L}}(\hat{\phi})=\hat{\phi}$ then $\mathscr{L}(\Pi(\hat{\phi}))=\Pi(\hat{\phi})$.

Our main result on the spectral properties of $\widehat{\mathscr{L}}$ follows:

Proposition 4.10 (Essential spectral radius of $\widehat{\mathscr{L}}$ ). - Let $f$ be an S-unimodal $\left(\lambda_{c}, H_{0}\right)$-Collet-Eckmann map satisfying the Benedicks-Carleson condition (3) for $\mathscr{G}=8$, with a non-preperiodic critical point. Let $\lambda$ satisfy (70). Then the operator $\widehat{\mathcal{L}}$ is bounded on $\mathscr{B}$, with 
spectral radius equal to 1 . The dual of $\widehat{\mathscr{L}}$ fixes the measure $\nu$ defined after (73). Let $\rho$ satisfy (26) and let $\sigma>1$ be the constant from Lemma 3.1, and set

$$
\Theta_{0}:=\min \left(\frac{\lambda_{c}^{1 / 2}}{e^{4 \gamma} \lambda}, \lambda, \sigma, \rho\right)>1 .
$$

The essential spectral radius of $\widehat{\mathscr{L}}$ on $\mathcal{B}$ is bounded by $\Theta_{0}^{-1}$.

Proof. - Let $c(\delta)$ be the constant from Lemma 3.1.

For $\hat{\psi} \in \mathscr{B}$, our assumptions on the $\xi_{j}$ ensure that $(\widehat{\mathscr{L}}(\hat{\psi}))_{k} \in W_{1}^{1}$ for all $k \geq 1$, with $(\widehat{\mathscr{L}}(\hat{\psi}))_{k}$ supported in the desired interval, and that $(\widehat{\mathscr{L}}(\hat{\psi}))_{0}$ is supported in the desired interval.

Note that for any interval $U$ (not necessarily containing the support of $\psi_{j}$ ), using the Sobolev embedding again,

$$
\sup _{U}\left|\psi_{j}\right| \leq \min \left(C\left\|\psi_{j}^{\prime}\right\|_{L^{1}}, \int_{U}\left|\psi_{j}^{\prime}\right| d x+\left|U^{-1} \int_{U}\right| \psi_{j} \mid d x\right) .
$$

Since $\xi_{\ell}$ is unimodal if it is not $\equiv 1$, for each $\ell \geq 1$ there exist $v_{\ell}>u_{\ell}$ in $B_{\ell}$ so that, setting $J_{\ell}=\left\{x \in \operatorname{supp}\left(\psi_{j}\right) \mid x \leq u_{\ell}\right\} \cup\left\{x \in \operatorname{supp}\left(\psi_{\ell}\right) \mid x \geq v_{\ell}\right\}$,

$$
\begin{aligned}
\int_{B_{\ell}}\left|\xi_{\ell}^{\prime} \psi_{\ell}\right| d x & =\int_{x \leq u_{\ell}} \xi_{\ell}^{\prime}\left|\psi_{\ell}\right| d x-\int_{x \geq v_{\ell}} \xi_{\ell}^{\prime}\left|\psi_{\ell}\right| d x \leq\left(\xi_{\ell}\left(u_{\ell}\right)+\xi_{\ell}\left(v_{\ell}\right)\right) \sup _{J_{\ell}}\left|\psi_{\ell}\right| \\
& \leq 2 \sup _{J_{\ell}}\left|\psi_{\ell}\right|
\end{aligned}
$$

(this can be viewed as the analogue of the " 2 sup sup" boundary term in [15]). Therefore, for all $k \geq 1$, using also (80),

$$
\left\|(\widehat{\mathscr{L}}(\hat{\psi}))_{k}^{\prime}\right\|_{L^{1}} \leq \frac{3 C}{\lambda}\left\|\psi_{k-1}^{\prime}\right\|_{L^{1}}
$$

More generally, for $1 \leq n \leq k$,

$$
\left\|\left(\widehat{\mathcal{L}}^{n}(\hat{\psi})\right)_{k}^{\prime}\right\|_{L^{1}} \leq \frac{3 C n}{\lambda^{n}}\left\|\psi_{k-n}^{\prime}\right\|_{L^{1}}
$$

If $\left|\psi_{k}(y)\right|>0$ then $\left|f^{j}(y)-c_{j}\right| \leq e^{-\beta_{1} j}$ for all $j \leq k$. If $\xi_{k}\left(f_{ \pm}^{-(k+1)}(x)\right)<1$ then $\left|x-c_{k+1}\right| \geq e^{-\beta_{2}(k+1)}$. Thus, changing variables in the integrals, using (81) for the terms involving $\xi_{k}^{\prime}$ for $k \geq 0$, and recalling (36) from Lemma 3.4, as well as (66) and (67) from Lemma 4.1, we see that $(\widehat{\mathscr{L}}(\hat{\psi}))_{0}$ belongs to $W_{1}^{1}$ and

$$
\begin{array}{rl}
\left\|(\widehat{\mathcal{L}}(\hat{\psi}))_{0}^{\prime}\right\|_{L^{1}} \leq C & c(\delta)^{-1}\left(\left\|\psi_{0}^{\prime}\right\|_{L^{1}}+\left\|\psi_{0}\right\|_{L^{1}}+\sup \left|\psi_{0}\right|\right) \\
& +\sum_{k \geq H(\delta)} \frac{\lambda^{k} e^{2 \gamma k}}{\left|\left(f^{k+1}\right)^{\prime}\left(c_{1}\right)\right|^{1 / 2}}\left(\left\|\psi_{k}^{\prime}\right\|_{L^{1}}+\sup \left|\psi_{k}\right|+\left\|\psi_{k}\right\|_{L^{1}}\right) .
\end{array}
$$

In view of (70) and (80), we have proved that $\widehat{\mathscr{L}}$ is bounded on $\mathscr{B}$. (The claim on the spectral radius will be proved below.)

Observe that $\sum_{k} \int_{B_{k}}|\hat{\psi}(x, k)| w(x, k) d x$ is finite if $\hat{\psi} \in \mathscr{B}$ (just recall that $\left|B_{k}\right| \leq 2 e^{-\beta_{1} k} \leq 2 e^{-3 \gamma k / 2}$ and use the bound $\lambda<e^{3 \gamma / 2}$ from (70)). So $\nu$ is an element of the dual of $\mathscr{B}$. The fact that $\widehat{\mathscr{L}}^{*}(\nu)=\nu$ can easily be proved using the change of 
variables formula. Indeed,

$$
\begin{gathered}
\int \widehat{\mathscr{L}}(\hat{\psi}) d \nu=\int_{B_{0}} \widehat{\mathscr{L}}(\hat{\psi})(y, 0) d y+\sum_{k \geq 0} \int_{B_{k+1}} \widehat{\mathscr{L}}(\hat{\psi})(y, k+1) w(y, k+1) d y \\
=\sum_{j \geq 0, \varsigma \in\{+,-\}} \int \frac{\lambda^{j}}{\left|\left(f^{j+1}\right)^{\prime} f_{\varsigma}^{-(j+1)}(y)\right|} \psi_{j}\left(f_{\varsigma}^{-(j+1)}(y)\right)\left(1-\xi_{j}\right)\left(f_{\varsigma}^{-(j+1)}(y)\right) d y \\
\quad+\sum_{k \geq 0} \int \frac{1}{\lambda} \psi_{k}(x) \xi_{k}(x) w(x, k+1) d x \\
=\sum_{j \geq 0} \int \psi_{j}(x)\left(1-\xi_{j}\right)(x) w(x, j) d x+\sum_{k \geq 0} \int \psi_{k}(x) \xi_{k}(x) w(x, k) d x .
\end{gathered}
$$

Note for further use that $\widehat{\mathscr{L}}^{*}(\nu)=\nu$ implies

$$
\nu\left(\left|\widehat{\mathscr{L}}^{N}(\hat{\psi})\right|\right) \leq \nu\left(\widehat{\mathscr{L}}^{N}(|\hat{\psi}|)\right)=\nu(|\hat{\psi}|) .
$$

We next estimate the spectral and essential spectral radii of $\widehat{\mathscr{L}}$ on $\mathscr{B}$. Using (81) and the overlap control of fuzzy intervals, it is not very difficult (see Appendix B) to adapt the proof of $\left[15\right.$, Sublemma] to show inductively that for any $\Theta<\Theta_{0}$, there exists $C$, and for all $n$ there exists $C(n)$, so that

$$
\left\|\left(\widehat{\mathcal{L}}^{n}(\hat{\psi})\right)_{0}^{\prime}(x)\right\|_{L^{1}(I)} \leq C \Theta^{-n}\|\hat{\psi}\|_{\mathcal{B}}+C(n)\|\hat{\psi}\|_{\mathscr{B}^{L^{1}}} .
$$

Recalling (83), and using (86), up to slightly decreasing $\Theta$, one then finds $C^{\prime}$ so that for all $n \geq 1$ (see the proof of [15, Variation Lemma])

$$
\left\|\hat{\mathscr{L}}^{n}(\hat{\psi})\right\|_{\mathscr{B}} \leq C^{\prime} \Theta^{-n}\|\hat{\psi}\|_{\mathscr{B}}+C^{\prime}\|\hat{\psi}\|_{\mathscr{B}^{L^{1}}} .
$$

Since (71) implies that the length of the support of $\psi_{k}$ is (much) smaller than $\lambda^{-2 k}$, we find $\|\hat{\psi}\|_{\mathcal{B}^{L^{1}}} \leq\|\hat{\psi}\|_{\mathcal{B}^{L^{1}}}+C \lambda^{-M}\|\hat{\psi}\|_{\mathscr{B}^{W_{1}^{1}}}$ for all $M \geq 1$ (we used again the Sobolev embedding to estimate the supremum by the $W_{1}^{1}$ norm). The bound (88) implies that the spectral radius of $\widehat{\mathscr{L}}$ on $\mathscr{B}$ is at most one, and thus equal to one.

Finally, since Rellich-Kondrachov implies that $\mathscr{B}^{W_{1}^{1}}$ is compactly included in $\mathscr{B}^{L^{1}}$ (the total length of the tower is bounded, even up to $\lambda^{k}$-expansion at level $k$ ), the Lasota-Yorke estimate (88) together with Hennion's theorem [24] give the claimed bound on essential spectral radius of $\widehat{\mathscr{L}}$ on $\mathscr{B}=\mathscr{B}^{W_{1}^{1}}$. This ends the proof of Proposition 4.10.

We next state further spectral properties of $\widehat{\mathscr{L}}$.

Proposition 4.11 (Maximal eigenvalue of $\widehat{\mathscr{L}}$ ). - Let $f$ be an $S$-unimodal $\left(\lambda_{c}, H_{0}\right)$-Collet-Eckmann map satisfying the Benedicks-Carleson condition (3) for $\mathscr{G}=8$, with a nonpreperiodic critical point.

The maximal eigenvalue 1 is a simple eigenvalue of $\widehat{\mathcal{L}}$, for a nonnegative eigenvector $\hat{\phi}$. If $\nu(\hat{\phi})=1$, then $\phi:=\Pi(\hat{\phi})$ is the density of the unique absolutely continuous $f$-invariant probability measure. Finally, if $f$ is $C^{4}$ and the Benedicks-Carleson condition (3) holds for $\mathscr{G}=14$, then one can choose the parameter $\lambda$ so that $\hat{\phi}_{0} \in W_{1}^{2}$. 
Note that $\sup _{k}\|\hat{\phi}\|_{W_{1}^{2}}=\infty$, since otherwise $\sup _{k}\left\|\hat{\phi}_{k}\right\|_{C^{1}}<\infty$ so that $\sup \left|\hat{\phi}_{k}\right|<C\left|\operatorname{supp}\left(\hat{\phi}_{k}\right)\right|$, which is impossible since $\sup \left|\hat{\phi}_{k}\right|=\lambda^{-k}\left|\hat{\phi}_{0}(c)\right| \neq 0$.

Proof. - By Proposition 4.10, the essential spectral radius of $\widehat{\mathscr{L}}$ on $\mathscr{B}$ is strictly smaller than one and the spectral radius is equal to 1 . The fact that 1 is a simple eigenvalue for a nonnegative eigenvector then follows from standard arguments (see, e.g., [15, Corollaries 1,2] or [59, Propositions 5.13, 5.14]). The normalization $\nu(\hat{\phi})=1$ implies that $\int_{I} \phi d x=$ $\int_{I} \Pi(\hat{\phi}) d x=1$. Recalling (78), we get that $\mathscr{L}(\Pi(\hat{\phi}))=\phi$ so that $\Pi(\hat{\phi}) \in L^{1}$ is indeed the invariant density of $f$ (which is known to be unique and ergodic).

It only remains to show that, under a stronger Benedicks-Carleson condition, $\hat{\phi}_{0} \in W_{1}^{2}$ if $f$ is $C^{4}$. For this, take $\hat{\psi}$ so that $\hat{\psi}_{k}=0$ for all $k \geq 1$ and $\hat{\psi}_{0}$ is $C^{\infty}$, of Lebesgue average 1 (we can even take $\hat{\psi}_{0}$ constant in a neighborhood of $\left[c_{2}, c_{1}\right]$ ), and use that $\widehat{\mathcal{L}}^{n}(\hat{\psi})$ converges to $\hat{\phi}$ in the $\mathscr{B}^{W_{1}^{1}}$ norm (exponentially fast) as $n \rightarrow \infty$. We claim that $\left\|\left(\widehat{\mathcal{L}}^{n}(\hat{\psi})\right)_{0}\right\|_{W_{1}^{3}} \leq C$ for all $n$, up to a suitable modification of the conditions on $\lambda$ in (70). Adapting the proof of (60), one shows $\sup _{x \in f^{k}\left(I_{k}\right)}\left|\partial_{x}^{3} \frac{1}{\left|\left(f^{k}\right)^{\prime}\left(f_{ \pm}^{-k}(x)\right)\right|}\right| \leq C \frac{e^{7 \gamma k}}{\left|\left(f^{k-1}\right)^{\prime}\left(c_{1}\right)\right|^{1 / 2}}$. Then, in view of (3) for $\mathscr{G}=14$, one can exploit (in addition to the properties already used in the proof of the Lasota-Yorke estimates for the $W_{1}^{1}$ norm in Proposition 4.10) the conditions on $\xi_{k}^{\prime \prime}, \xi_{k}^{\prime \prime \prime}$ in (75) to adapt (152) in Appendix B (noting also that $\hat{\psi} \mid \omega=0$ if the interval $\omega$ is in some level $E_{k}$ with $k>0)$. Note that (81) is not needed, since we only look at the component of $\widehat{\mathscr{L}}^{n}(\hat{\psi})$ at level 0 . Details are straightforward and left to the reader. (We do not claim that the factor $\mathscr{G}=14$ in (3) is optimal. In any case, we shall need to work with the stronger TSR condition soon.) To conclude, use that if a sequence converging to $\hat{\phi}_{0}$ in $W_{1}^{1}(I)$ has bounded $W_{1}^{3}(I)$ norms then $\hat{\phi}_{0} \in W_{1}^{2}(I)$ by Rellich-Kondrakov.

\subsection{Truncated transfer operators $\widehat{\mathscr{L}}_{M}$}

We introduce for each $M \geq 0$ the truncation operator $\mathcal{J}_{M}$ defined by

$$
\mathcal{T}_{M}(\hat{\psi})_{k}= \begin{cases}\psi_{k} & k \leq M \\ 0 & k>M .\end{cases}
$$

Clearly, $\left\|\mathcal{J}_{M}\right\|_{\mathscr{B}^{W_{1}^{r}}} \leq 1$ for all integers $r \geq 0$ and $\left\|\mathcal{J}_{M}\right\|_{\mathscr{B}^{L^{1}}} \leq 1$. We consider the operator defined by

$$
\widehat{\mathscr{L}}_{M}=\mathcal{T}_{M} \widehat{\mathscr{L}} \mathcal{T}_{M} .
$$

Using the results of Keller and Liverani [29], we shall prove the following lemma:

Lemma 4.12 (Spectrum of the truncated operators). - Let $f$ be an S-unimodal $\left(\lambda_{c}, H_{0}\right)$-Collet-Eckmann map satisfying the Benedicks-Carleson condition (3) for $\mathscr{G}=8$, with a non-preperiodic critical point. Recall $\Theta_{0}$ from (79).

The essential spectral radius of $\widehat{\mathscr{L}}_{M}$ acting on $\mathscr{B}$ is not larger than $\Theta_{0}^{-1}<1$.

In addition, there exists $M_{0} \geq 1$ so that for all $M \geq M_{0}$ the operator $\widehat{\mathcal{L}}_{M}$ has a real nonnegative maximal eigenfunction $\hat{\phi}_{M}$, for an eigenvalue $\kappa_{M}>\Theta_{0}^{-1}$, the dual operator of $\widehat{\mathscr{L}}_{M}$ has a nonnegative maximal eigenfunction $\nu_{M}$, and, setting

$$
\tau_{M}=e^{3 \gamma M}\left|\left(f^{M}\right)^{\prime}\left(c_{1}\right)\right|^{-1 / 2}<1
$$


for any $\eta<1$ there exists $C>0$ so that, normalizing by $\nu(1)=\nu_{M}(1)$ and $\int \hat{\phi}_{M} d \nu_{M}=1$, we have

$$
\left\|\hat{\phi}-\hat{\phi}_{M}\right\|_{\mathscr{B}^{L^{1}}} \leq C \tau_{M}^{\eta},\left\|\nu-\nu_{M}\right\|_{\left(\mathscr{B}^{\left.W_{1}^{1}\right)^{*}}\right.} \leq C \tau_{M}^{\eta},\left|\kappa_{M}-1\right| \leq C \tau_{M}^{\eta} .
$$

If $f$ is $C^{4}$ and the Benedicks-Carleson condition (3) holds for $\mathscr{G}=14$, we may choose $\lambda>1$ so that $\sup _{M}\left\|\hat{\phi}_{M, 0}\right\|_{W_{1}^{2}}<\infty$.

Proof. - The claim about the essential spectral radius can be obtained by going over the proof of Proposition 4.10 and checking that it applies to $\widehat{\mathscr{L}}_{M}$, and that the constants are uniform in $M$. The reader is invited to do this, and to check that we have the following uniform Lasota-Yorke estimates for $\widehat{\mathscr{L}}$ and $\widehat{\mathscr{L}}_{M}$ : There exists $C \geq 1$ so that for all $N$ and all $M$

$$
\max \left(\left\|\widehat{\mathcal{L}}^{N}(\hat{\psi})\right\|_{\mathscr{B}^{W 1}},\left\|\widehat{\mathcal{L}}_{M}^{N}(\hat{\psi})\right\|_{\mathscr{B}^{W_{1}^{1}}}\right) \leq C \Theta^{-N}\|\hat{\psi}\|_{\mathscr{B}^{W_{1}^{1}}}+C\|\hat{\psi}\|_{\mathscr{B}^{L^{1}}}
$$

and (recall (86) and note that $\left.\nu\left(\left|\widehat{\mathscr{L}}_{M}^{N}(\hat{\psi})\right|\right) \leq \nu\left(\widehat{\mathscr{L}}_{M}^{N}(|\hat{\psi}|)\right) \leq \nu\left(\widehat{\mathscr{L}}^{N}(|\hat{\psi}|)\right)=\nu(|\psi|)\right)$

$$
\left\|\widehat{\mathscr{L}}^{N}\right\|_{\mathscr{B}^{L^{1}}} \leq 1, \quad\left\|\left(\widehat{\mathscr{L}}_{M}\right)^{N}\right\|_{\mathscr{B}^{L^{1}}} \leq 1, \forall M, \forall N
$$

Finally, there exists $C$ so that for all large enough $M$

$$
\left\|\left(\widehat{\mathcal{L}}-\widehat{\mathscr{L}}_{M}\right)(\hat{\psi})\right\|_{\mathcal{B}^{L^{1}}} \leq C \tau_{M}\|\hat{\psi}\|_{\mathcal{B}^{W_{1}^{1}}} .
$$

The last inequality is an easy consequence of

$$
\left\|\left(\mathrm{id}-\mathcal{J}_{M}\right) \hat{\psi}\right\|_{\mathcal{B}^{L^{1}}} \leq C \tau_{M}\|\hat{\psi}\|_{\mathscr{B}^{W_{1}^{1}}}
$$

which follows from Lemma 4.1 and Proposition 3.7, since $\left|\sup \psi_{k}\right| \leq C\left\|\psi_{k}^{\prime}\right\|_{L^{1}}$. The bounds (91) for $\eta \in(0,1)$ then follow from [29, Theorem 1, Corollary 1].

It follows from what has been done up to now and [29] that $\sup _{M}\left\|\hat{\phi}_{M}\right\|_{\mathcal{B}^{W_{1}^{1}}}<\infty$. For the last claim of Lemma 4.12, we proceed like in the analogous statement of Proposition 4.11, and get uniform bounds in $M$.

\section{Topological invariance and uniformity of constants for various recurrence conditions}

It is well-known [40] that the Collet-Eckmann property is an invariant of topological conjugacy, and the fact that $\lambda_{c}\left(f_{t}\right)$ can be estimated uniformly in $t$ for a smooth deformation $f_{t}$ of $f_{0}$ is explained, e.g., in [11, Appendix]. Our argument requires more: We need a BenedicksCarleson condition (3) for $\mathscr{G}=8$ or $\mathscr{G}=14$ and uniform estimates on the constants

$$
\lambda_{c}\left(f_{t}\right), H_{0}\left(f_{t}\right), \gamma\left(f_{t}\right), \text { and also } \sigma\left(f_{t}\right), C_{1}\left(f_{t}\right), c\left(\delta, f_{t}\right), \rho\left(f_{t}\right)
$$

(recall Lemma 3.1), as $t$ varies. The constant $\sigma\left(f_{t}\right)$ is bounded away from 1 uniformly in small $t$, by the proof of [36, Theorem III.3.3], in particular the choice of $m$ and $\lambda$ there, and noting that all $f_{t}$ have only repelling periodic orbits and are $S$-unimodal. However, if $f_{t}$ is a smooth deformation of a Benedicks-Carleson $S$-unimodal map, we do not know how to estimate $\gamma\left(f_{t}\right)$ in general.

Lemma 5.8, the main result of this section, is proved in Subsection 5.1: It says that all constants in (93) are uniform, for deformations $f_{t}$ which satisfy the TSR condition (5). In Subsection 5.2, we exploit a consequence of this uniformity which will play an important 
part in the proof of Theorem 2.13: If $f_{t}$ is a deformation, one can use the same lower part of the tower for all operators $\widehat{\mathscr{L}}_{t}$ with $|t| \leq t_{0}$, up to some level depending on $t_{0}$.

In order to apply directly the results of Nowicki [38], we shall assume that $f$ is symmetric, i.e.,

$$
f(x)=f(-x)
$$

\subsection{Uniformity of constants}

Recall that our definition of $S$-unimodal includes the condition $f^{\prime \prime}(c) \neq 0$, that is, all our $S$-unimodal maps are quadratic. Let $R_{f}(x)$ be the function from (4) in the definition of the TSR condition.

Proposition 5.1 (Uniform Collet-Eckmann condition [32]). - Let $f_{0}$ be a $C^{3} S$-unimodal map satisfying the topological slow recurrence condition (5). Then there exist $\lambda_{c}>1$, $\underline{\kappa}>0, K>0$, and $\epsilon>0$ such that for every $S$-unimodal map $f$ in the topological class of $f_{0}$ such that $\left|f-f_{0}\right|_{C^{3}}<\epsilon$, we have

$$
R_{f}\left(f^{j}(c)\right) \geq-\underline{\kappa} \ln \left|f^{j}(c)-c\right|, \quad \forall j \geq 0,
$$

and

$$
\left|\left(f^{j}\right)^{\prime}(f(c))\right| \geq K \lambda_{c}^{j}, \quad \forall j \geq 0 .
$$

Proof. - Except for the explicit statement on the dependence of $\underline{\kappa},(95)$ is Lemma 2 in [32]. We say that $f \in V(D, L, \theta)$ if

$$
D_{f}=\max _{x \in I}\left|f^{\prime}(x)\right|<D, L_{f}=\sup _{x \in I} \frac{|f(x)-f(c)|}{|x-c|^{2}}<L, \theta_{f}=\sup _{f(x)=f(y)} \frac{|x-c|}{|y-c|}<\theta .
$$

For $\epsilon$ small enough, we have $f \in V(D, L, \theta)$, with $D=2 D_{g_{0}}, L=2 L_{f_{0}}$ and $\theta=2 \theta_{f_{0}}$. The proof of Lemma 2 relies on Sublemmas 2.1 and 2.2 in [32]. The constants $C$ and $\underline{\kappa}$ in [32, Sublemma 2.1] depend only on $D, L$ and $\theta$. The constant $N_{\varepsilon}$ in [32, Sublemma 2.2] depends only on the topological class of $f$. In the proof of Lemma 2 in [32], since $f$ has a unique critical point we can take $\delta_{0}=|I|$ and $N_{0}=1$ in (5) and (6) of [32]. Moreover, we can find $\epsilon>0$ such that

$$
\inf _{\left|f-f_{0}\right|_{C^{3}}<\epsilon} \min \left\{\left|f^{i}(c)-c\right| \text { s.t. } 0<i \leq \max \left\{N_{0}, N_{\varepsilon}\right\}\right\}>0 .
$$

This shows (95).

Except for the explicit statement on the dependence of $K$ and $\lambda_{c}$, (96) is Corollary 5.1 in [32]. The proof of this result relies on (95) above, and on Lemmas 3, 4, 5 and Sublemma 5.1 in [32]. The estimates obtained in Lemma 3 depend only on the topological class of $f$. Given $T>0$, we can find $\epsilon>0$ such that

$$
\inf _{\left|f-f_{0}\right|_{C^{3}}<\epsilon} \min \left\{|x-y| \text { s.t. } x \neq y, x, y \in\left\{f^{i}(c)\right\}_{i \leq T} \cup\left\{z: f^{i}(z)=c\right\}_{i \leq T}\right\}>0,
$$

so we can see from the proof of [32, Lemma 4] that there exists $\gamma(T)$ that satisfies the estimates obtained in Lemma 4 for every $S$-unimodal map $f$ in the topological class of $f_{0}$ satisfying $\left|f-f_{0}\right|_{C^{3}}<\epsilon$. Sublemma 5.1 in [32] follows directly from Lemmas 3 and 4 for every $f$ satisfying the same conditions, with the same constants $\eta$ and $\gamma$. Finally the proof of 
the estimates in Lemma 5 in [32] depends only on the topological class of $f$, estimates in Sublemma 2.2, (95) above and $D$.

Proposition 5.2 (Uniform Benedicks-Carleson type conditions)

Let $f_{0}$ be an $S$-unimodal map satisfying the topological slow recurrence condition (5). Then for every $\gamma>0$ there exist $H_{0}>0$ and $\epsilon>0$ such that for every $S$-unimodal map $f$ in the topological class of $f_{0}$ such that $\left|f-f_{0}\right|_{C^{3}}<\epsilon$, we have

$$
\left|f^{k}(c)-c\right| \geq e^{-\gamma k}, \quad \forall k \geq H_{0} .
$$

Proof. - Let $\underline{\kappa}$ and $\epsilon$ be as in Proposition 5.1. By (5) we can choose $m_{0}$ such that

$$
\limsup _{n \rightarrow \infty} \frac{1}{n} \sum_{\substack{1 \leq j \leq n \\ R_{f_{0}}\left(f_{0}^{j}(c)\right) \geq m_{0}}} R_{f_{0}}\left(f_{0}^{j}(c)\right)<\underline{\kappa} \gamma / 2,
$$

so for $n_{0}$ large enough

$$
\frac{1}{n} \sum_{\substack{1 \leq j \leq n \\ R_{f_{0}}\left(f_{0}^{j}(c)\right) \geq m_{0}}} R_{f_{0}}\left(f_{0}^{j}(c)\right)<\underline{\kappa} \gamma, \forall n \geq n_{0} .
$$

Consequently, we have the same estimate for every map $f$ topologically conjugate to $f_{0}$, that is

$$
\frac{1}{n} \sum_{\substack{1 \leq j \leq n \\ R_{f}\left(f^{j}(c)\right) \geq m_{0}}} R_{f}\left(f^{j}(c)\right)<\underline{\kappa} \gamma, \forall n \geq n_{0} .
$$

In particular, if $R_{f}\left(f^{k}(c)\right) \geq m_{0}$ and $k \geq n_{0}$, we have

$$
\frac{R_{f}\left(f^{k}(c)\right)}{k}<\underline{\kappa} \gamma
$$

so by (95) we obtain

$$
-\frac{\ln \left|f^{k}(c)-c\right|}{k}<\gamma,
$$

so $\left|f^{k}(c)-c\right| \geq e^{-\gamma k}$. Since $c$ is not periodic for $f_{0}$, we can find $\eta, \epsilon>0$ such that for each $S$-unimodal map $f$ such that $\left|f-f_{0}\right|_{C^{3}}<\epsilon$ and for every $x \in(c-\eta, c+\eta)$ we have $\left|f^{i}(x)-c\right|>0$ for $1 \leq i \leq 2 m_{0}$. In particular, dist $\left(\Omega_{f}, c\right)>\eta$, where

$$
\Omega_{f}=\left\{x \in I: R_{f}(x)<m_{0}\right\} .
$$

Let $H_{0}>n_{0}$ be large enough such that $\eta>e^{-\gamma H_{0}}$. Then $\left|f^{k}(c)-c\right| \geq e^{-\gamma k}$ for every $k \geq H_{0}$.

We are going to use some results by Nowicki [38]. An interval $\left[a_{1}, a_{2}\right]$ is a nice interval if $c \in\left(a_{1}, a_{2}\right)$ and $f^{j}\left(a_{i}\right) \notin\left(a_{1}, a_{2}\right)$, for every $j \geq 1$ and $i=1,2$. We say that an interval $(c, b)$ is a $*(n)$ interval if $f^{n}$ is a diffeomorphism on $(c, b)$ and $f^{n}(b)=c$.

Proposition 5.3 (Lemma 9 and Proposition 11 in [38]). - Let $(c, b)$ be an $*(n)$ interval of a symmetric $S$-unimodal map $f$ satisfying (96). Then $\left|f^{n}(c)-f^{n}(b)\right|>|c-b|$. Furthermore

$$
\left|f^{n}(c)-f^{n}(b)\right| \geq K_{1} \lambda_{c}^{n / 4}|c-b|,
$$

where $K_{1}=(K m / 4 M)^{1 / 2}$, where $K$ is as in (96), and $m$ and $M$ satisfy $m|x-c| \leq\left|f^{\prime}(x)\right| \leq$ $M|x-c|$. 
Proposition 5.4 (Proposition 13 in [38]). - Let $f$ be a symmetric S-unimodal map satisfying (96). Let $b \in[-1,1]$ be such that $f^{n}(b)=c$. Then

$$
\left|\left(f^{n}\right)^{\prime}(b)\right| \geq \rho^{n}
$$

for every ${ }^{(19)}$

$$
\rho \leq \min \left(\inf _{n} \inf _{(c, b) \text { an } *(\mathrm{n}) \text { interval }}\left|\frac{f^{n}(c)-f^{n}(b)}{c-b}\right|^{1 / n},\left|f^{\prime}(-1)\right|^{1 / 2}\right) .
$$

(The right-hand side above is $>1$ by Proposition 5.3.)

Proposition 5.5. - Let $f_{0}$ be an S-unimodal map satisfying the topological slow recurrence condition (5). Then for every $\beta \in(0,1)$ there exist $\epsilon, \delta>0$ and $\tilde{K}>0$ with the following property: Let $f$ be a symmetric $S$-unimodal map in the topological class of $f_{0}$ such that $\left|f-f_{0}\right|_{C^{3}}<\epsilon$, let $[-q, q] \subset[-\delta, \delta]$ be a nice interval for $f$, and let $x \in[-1,1] \backslash[-q, q]$ be such that $f^{n}(x) \in[-q, q]$ for some $n \geq 1$. Define

$$
n_{0}(x)=\min \left\{n \geq 1 \text { s.t. } f^{n}(x) \in[-q, q]\right\} .
$$

Then there exist intervals $I_{n_{0}(x)} \subset J_{n_{0}(x)}$, with $x \in I_{n_{0}(x)}$, such that

1. For every $y \in I_{n_{0}(x)}$ we have $n_{0}(y)=n_{0}(x)$ and $f^{n_{0}(x)} I_{n_{0}(x)}=[-q, q]$.

2. The map $f^{n_{0}(x)}: J_{n_{0}(x)} \rightarrow f^{n_{0}(x)} J_{n_{0}(x)}$ is a diffeomorphism, and each connected component of $f^{n_{0}(x)} J_{n_{0}(x)} \backslash\{c\}$ is larger than $\tilde{K} q^{\beta}$.

Proof. - The existence of $I_{n_{0}(x)}$ satisfying Claim 1 follows from the fact that $[-q, q]$ is a nice interval. Let $[a, b]=J_{n_{0}(x)} \supset I_{n_{0}(x)}$ be the largest interval such that $f^{n_{0}(x)}$ is a diffeomorphism on $(a, b)$. In particular there are $n_{a}, n_{b}<n_{0}(x)$ such that $f^{n_{a}}(a) \in\{1,-1, c\}$ and $f^{n_{b}}(b) \in\{1,-1, c\}$. Suppose $f^{n_{b}}(b)=c$. We are going to show that $\left|f^{n_{0}(x)} b-c\right| \geq \tilde{K} q^{\beta}$. The proof of the analogous statement for $a$ is similar. By Claim 1 there is $d \in I_{n_{0}(x)}$ such that $f^{n_{0}(x)}(d)=c$ and, moreover, $f^{n_{b}}(d) \notin[-q, q]$, so either $[-q, c] \subset\left[f^{n_{b}}(d), c\right]=f^{n_{b}}[d, b]$ or $[c, q] \subset\left[c, f^{n_{b}}(d)\right]=f^{n_{b}}[b, d]$. Since $\left(f^{n_{b}}(d), c\right)$ is a $*\left(n_{0}(x)-n_{b}\right)$ interval [38], by Proposition 5.3 and Proposition 5.1, we have

$$
\left|f^{n_{0}(x)}(b)-c\right|=\left|f^{n_{0}(x)-n_{b}}(c)-c\right| \geq K_{1} \lambda_{c}^{\left(n_{0}(x)-n_{b}\right) / 4}\left|c-f^{n_{b}}(d)\right| \geq K_{1} \lambda_{c}^{\left(n_{0}(x)-n_{b}\right) / 4} q,
$$

where $K_{1}$ is uniform on a $C^{3}$ neighborhood of $f_{0}$. Choose

$$
0<\gamma<\frac{\beta \ln \lambda_{c}}{4(1-\beta)}
$$

Reducing this neighborhood, if necessary, we have by Proposition 5.2 that

$$
\left|f^{n_{0}(x)}(b)-c\right|=\left|f^{n_{0}(x)-n_{b}}(c)-c\right| \geq K e^{-\gamma\left(n_{0}(x)-n_{b}\right)} .
$$

We have two cases. If $n_{0}(x)-n_{b}>-4(1-\beta) \ln q / \ln \lambda_{c}$ then, by (98), we easily obtain $\left|f^{n_{0}(x)}(b)-c\right| \geq K_{1} q^{\beta}$. Otherwise $n_{0}(x)-n_{b} \leq-4(1-\beta) \ln q / \ln \lambda_{c}$, so by (99), we get

$$
\left|f^{n_{0}(x)}(b)-c\right|=\left|f^{n_{0}(x)-n_{b}}(c)-c\right| \geq K e^{-\gamma\left(n_{0}(x)-n_{b}\right)} \geq K e^{-\frac{\beta \ln \lambda_{c}}{4(1-\beta)}\left(n_{0}(x)-n_{b}\right)} \geq K q^{\beta} .
$$

(19) Note that $\rho$ is called $\lambda_{T}$ in [38]. 
Choose $\tilde{K}=\min \left(K, K_{1}\right)$. If $\left|f^{n_{b}}(b)\right|=1$, then $f^{n_{0}(x)}(b)=-1$. Choose $\delta$ such that $\delta^{-\beta} \geq \tilde{K}$. Then

$$
\left|f^{n_{0}(x)}(b)-c\right|=1 \geq \tilde{K} \delta^{\beta} \geq \tilde{K} q^{\beta} .
$$

COROLlary 5.6 (Uniformity of $C_{1}$ and $\rho$ ). - Let $f_{0}$ be a symmetric S-unimodal map satisfying the topological slow recurrence condition (5). There exist $\rho>1$ and $\epsilon>0$ with the following property: For every $C_{1} \in(0,1)$ there exists $\delta>0$ so that, for every symmetric $S$-unimodal map $f$ in the topological class of $f_{0}$ such that $\left|f-f_{0}\right|_{C^{3}}<\epsilon$, and for every nice interval $[-q, q]$ of $f$ such that $q<\delta$, if $x \notin[-q, q]$ and $n \geq 1$ is the first entrance time of $x$ in $[-q, q]$, then

$$
\left|\left(f^{n}\right)^{\prime}(x)\right| \geq C_{1} \rho^{n}
$$

Proof. - By Proposition 5.1, we can find $\epsilon_{0}>0$ and $\rho>1$ such that Proposition 5.4 holds for every $f$ such that $\left|f-f_{0}\right|_{C^{3}}<\epsilon_{0}$, with $f$ in the topological class of $f_{0}$. Take $\beta=1 / 2$, and let $\epsilon<\epsilon_{0}, \delta$ be as in Proposition 5.5. Reducing $\delta$ if necessary, we have that if $q<\delta$ then each connected component of $f^{n}\left(J_{n}(x)\right) \backslash[-q, q]$ is far larger than $q$. In particular by the Koebe lemma

$$
\frac{\left(f^{n}\right)^{\prime}(z)}{\left(f^{n}\right)^{\prime}(w)}<C_{1}^{-1}, \forall z, w \in I_{n}(x) \text {. }
$$

But there exists $b \in I_{n}(x)$ such that $f^{n}(b)=c$, so $\left(f^{n}\right)^{\prime}(b) \geq \rho^{n}$. We conclude that $\left|\left(f^{n}\right)^{\prime}(x)\right| \geq C_{1} \rho^{n}$.

Finally, we shall need the following result:

Corollary 5.7 (Uniformity of $c(\delta)$ and $\sigma$ ). - Let $f_{0}$ be a symmetric S-unimodal map satisfying the topological slow recurrence condition (5). There exists ${ }^{(20)} \sigma>1$ such that for every $\delta>0$ there exist $c(\delta)>0$ and $\epsilon>0$ with the following property: For every symmetric $S$-unimodal map $f$ in the topological class of $f_{0}$ such that $\left|f-f_{0}\right|_{C^{3}}<\epsilon$, if $\left|f^{i}(x)\right|>\delta$ for $0 \leq i<n$ then

$$
\left|\left(f^{n}\right)^{\prime}(x)\right| \geq c(\delta) \sigma^{n}
$$

Proof. - By Proposition 5.1, we can find $\epsilon_{0}>0$ and $\rho>1$ such that Proposition 5.4 holds for every $f$ such that $\left|f-f_{0}\right|_{C^{3}}<\epsilon_{0}$, with $f$ in the topological class of $f_{0}$. Using the same argument as in Proposition 3.9 in [37], we can show that for every periodic point $q$ such that $f^{n}(q)=q$ we have $\left|\left(f^{n}\right)^{\prime}(q)\right| \geq \rho^{n}$. Note that since $c$ is recurrent by $f_{0}$, there exists a sequence of periodic points for $f_{0}$ converging to $c$. So given $\delta>0$ there exists a periodic point $p$ for $f_{0}$ such that $|p|<\delta$. Let $n_{0}$ be the prime period of $p$. There exists $\epsilon_{1}<\epsilon_{0}$ such that every map $f$ such that $\left|f-f_{0}\right|_{C^{3}}<\epsilon_{1}$ has an analytic continuation $p_{f}$ for $p$ such that $\left|p_{f}\right|<\delta$ and

$$
\eta_{p e r}=\inf _{\left|f-f_{0}\right|_{C^{3}<\epsilon_{1}}}\left|p_{f}\right|>0
$$

Without loss of generality, we can assume that $\left|f^{i}\left(p_{f}\right)\right| \geq\left|p_{f}\right|$ for every $i$. So $\left[-p_{f}, p_{f}\right]$ is a nice interval. Let $x \notin[-\delta, \delta]$ be such that $\left|f^{i}(x)\right|>\delta$ for $0 \leq i<n$. If $f^{n}(x) \in\left[-p_{f}, p_{f}\right]$ we can use Corollary 5.6 to conclude that $\left|\left(f^{n}\right)^{\prime}(x)\right| \geq C_{1} \rho^{n}$. So assume that $f^{n}(x) \notin\left[-p_{f}, p_{f}\right]$. Let $(a, b)$ be the largest interval such that $x \in(a, b)$ and $f^{i}(y) \notin\left[-p_{f}, p_{f}\right]$ for every

(20) The analogue of $\sigma$ is called $\lambda_{M}$ in [37].

$4{ }^{\text {e }}$ SÉRIE - TOME $45-2012-\mathrm{N}^{\circ} 6$ 
$0 \leq i \leq n$ and $y \in(a, b)$. In particular, $f^{n}$ is a diffeomorphism on $(a, b)$, and there exist $n_{a}, n_{b} \leq n$ such that $\left|f^{n_{a}}(a)\right|,\left|f^{n_{b}}(b)\right| \in\left\{\left|p_{f}\right|, 1\right\}$. Without loss of generality, we can assume that $\left|f^{i}(a)\right|,\left|f^{j}(b)\right| \notin\left\{\left|p_{f}\right|, 1\right\}$ for every $i<n_{a}, j<n_{b}$. If $f^{n_{a}}(a) \in\{-1,1\}$, then indeed $a \in\{-1,1\}$, so $\left|\left(f^{n}\right)^{\prime}(a)\right|=\left|f^{\prime}(-1)\right|^{n}$. We have a similar statement for $b$. Otherwise either $f^{n_{a}}(a)$ (respectively $f^{n_{b}}(b)$ ) or $-f^{n_{a}}(a)$ (respectively $-f^{n_{b}}(b)$ ) is a periodic point with period $n_{0}$. Then $n_{a}$ and $n_{b}$ are the first entry times of $a$ and $b$ in $\left[-p_{f}, p_{f}\right]$. By Corollary 5.6, we have

$$
\left|\left(f^{n_{a}}\right)^{\prime}(a)\right| \geq C_{1} \rho^{n_{a}} \text { and }\left|\left(f^{n_{b}}\right)^{\prime}(b)\right| \geq C_{1} \rho^{n_{b}} .
$$

Since $p_{f}$ is a periodic point of period $n_{0},\left|\left(f^{n_{0}}\right)^{\prime}\left(p_{f}\right)\right| \geq \rho^{n_{0}}$ and $f$ is symmetric and quadratic, we have

$$
\begin{aligned}
\left|\left(f^{n-n_{a}}\right)^{\prime}\left(f^{n_{a}}(a)\right)\right| & \geq \rho^{n-n_{a}-n_{0}} \min \left\{\left|f^{\prime}\left(f^{i}\left(p_{f}\right)\right)\right|, 0 \leq i<n_{0}\right\}^{n_{0}} \\
& \geq C^{n_{0}}\left|p_{f}\right|^{n_{0}} \rho^{n-n_{a}-n_{0}}
\end{aligned}
$$

so

$$
\left|\left(f^{n}\right)^{\prime}(a)\right| \geq C^{n_{0}}\left|p_{f}\right|^{n_{0}} \rho^{n-n_{0}} \geq C^{n_{0}} \eta_{p e r}^{n_{0}} \rho^{-n_{0}} \rho^{n}=c(\delta) \rho^{n} .
$$

We can obtain similarly $\left|\left(f^{n}\right)^{\prime}(b)\right| \geq c(\delta) \rho^{n}$. In any case

$$
\min \left(\left|\left(f^{n}\right)^{\prime}(a)\right|,\left|\left(f^{n}\right)^{\prime}(b)\right|\right) \geq \min (1, c(\delta)) \cdot \min \left(\rho,\left|f^{\prime}(-1)\right|\right)^{n} .
$$

By the minimum principle

$$
\left|\left(f^{n}\right)^{\prime}(x)\right| \geq \min (1, c(\delta)) \cdot \min \left(\rho,\left|f^{\prime}(-1)\right|\right)^{n} .
$$

So choose $\sigma=\min \left(\rho,\left|f^{\prime}(-1)\right|\right)>1$.

Summarizing the results of this section, we have proved:

Lemma 5.8 (Uniformity of constants in topological classes of TSR maps)

If $f_{0}$ is a symmetric $S$-unimodal $\left(\lambda_{c}\left(f_{0}\right), H_{0}\left(f_{0}\right)\right)$-Collet-Eckmann map satisfying topological slow recurrence (5), then for every $C_{1} \in(0,1)$ there exists $\lambda_{c} \in\left(1, \lambda_{c}\left(f_{0}\right)\right)$ so that for any $\gamma \in\left(0, \log \left(\lambda_{c}\right) / 14\right)$ there exists $H_{0}>H_{0}\left(f_{0}\right)$ so that for each $\rho \in\left(1, \lambda_{c}^{1 / 2}\right)$, there exist $\sigma>1$ and $\delta_{0}>0$ so that for every $\delta \in\left(0, \delta_{0}\right)$ there exist $c(\delta)=c_{f}(\delta)>0$ and $\epsilon>0$ so that the following holds for each symmetric $S$-unimodal map $f$ topologically conjugated to $f_{0}$ and so that $\left|f-f_{0}\right|_{C^{3}}<\epsilon$ :

The map $f$ is $\left(\lambda_{c}, H_{0}\right)$-Collet-Eckmann and satisfies (3) with $\mathscr{G}=14$ for the above given values of $\gamma, \lambda_{c}$, and $H_{0}$. For any $y \in I$, if $j \geq 0$ is minimal satisfying $\left|f^{j}(y)\right| \leq \delta$, then

$$
\left|\left(f^{j}\right)^{\prime}(y)\right| \geq C_{1} \rho^{j}
$$

for any $x \in I$, if $j \geq 1$ is such that $\left|f^{k}(x)\right|>\delta$ for all $0 \leq k<j$, then

$$
\left|\left(f^{i}\right)^{\prime}(x)\right| \geq c(\delta) \sigma^{i}, \forall 0 \leq i \leq j .
$$

Comparing the above result to Lemma 3.1 we emphasize that we do not claim that $\lambda_{c}$ can be taken arbitrarily close to $\lambda_{c}\left(f_{0}\right)$ or $H_{0}$ close to $H_{0}\left(f_{0}\right)$, where $\lambda_{c}\left(f_{0}\right), H_{0}\left(f_{0}\right)$ are the best possible constants for $f_{0}$. So the conclusion of Lemma 5.8 cannot be viewed strictly as a Benedicks-Carleson condition. (This is mostly because of the infimum in the right-hand side of (97) from Proposition 5.4.) However, this does not matter since we are assuming the much stronger TSR assumption in any case (see also (109) below), which implies that we can take $\gamma$ arbitrarily close to 0 after $\lambda_{c}$ has been fixed. The advantage of the notation 
introduced in Lemma 5.8 is that we can use the estimates from Sections 3 and 4 directly, with the same notation for the constants, for deformations of maps $f_{0}$ satisfying the assumptions of Lemma 5.8.

Finally, we give a long postponed proof:

Proof of Lemma 2.12. - We may assume that the critical point is not preperiodic. (If it is, the proof is much easier.)

The proof of Proposition 3.9 shows that for each fixed $s$ the supremum $\sup _{x}\left|\alpha_{s}(x)\right|$ may be estimated in terms of $c_{f_{s}}(\delta)^{-1}, \sup \left|v_{s}\right|, \operatorname{Lip} X_{s},\left(1-\sigma\left(f_{s}\right)^{-1}\right)^{-1},\left(1-\left(\rho\left(f_{s}\right)\right)^{-1}\right)^{-1}$, in the notation of Lemma 3.1. By Lemma 5.8, this implies (13).

Next, applying Lemma 2.10 to each $f_{s}$, we get

$$
\lim _{t \rightarrow 0} \frac{c_{k, s+t}-c_{k, s}}{t}=\alpha_{s}\left(h_{s}\left(c_{k}\right)\right)=\alpha_{s}\left(c_{k, s}\right) .
$$

In other words, $t \mapsto h_{t}\left(c_{k}\right)$ is differentiable on $[-\epsilon, \epsilon]$ (with $\epsilon$ independent of $k$ ), with derivative $\alpha_{s}\left(c_{k, s}\right)$.

Then, for each $k \geq 1$ and each $|t|<\epsilon$ the mean value theorem gives $s$ with $|s| \leq|t|$ so that

$$
\frac{c_{k, t}-c_{k}}{t}=\frac{h_{t}\left(c_{k}\right)-h_{0}\left(c_{k}\right)}{t}=\alpha_{s}\left(c_{k, s}\right) .
$$

\subsection{Transfer operators $\widehat{\mathscr{L}}_{t}, \widehat{\mathscr{L}}_{t, M}$ for a (TSR) smooth deformation $f_{t}$}

If $f_{t}$ is a $C^{1}$ one-parameter family of $S$-unimodal symmetric Collet-Eckmann maps $f_{t}$, with a non preperiodic critical point, Lemma 5.8 implies that all $f_{t}$ satisfy estimates for uniform parameters $\lambda_{c}$ and $H_{0}$, and satisfy the Benedicks-Carleson condition (3) for $\gamma>0$ and $\mathscr{G}=14$. We can associate a tower $\hat{f}_{t}: \hat{I}_{t} \rightarrow \hat{I}_{t}$ to each $f_{t}$, choosing small $\delta_{t}$ and intervals $B_{k, t}$ by using the parameters $\lambda_{c}, H_{0}, \gamma$ as in Subsection 3.1, replacing $c_{k}$ by $c_{k, t}$. Then, we can define spaces $\mathscr{B}_{t}^{W_{1}^{1}}$, and an operator $\widehat{\mathcal{L}}_{t}$ in Subsection 4.1, replacing $f^{k}$ by $f_{t}^{k}$ and $c_{k}$ by $c_{k, t}$ in (71) and the definition of $\xi_{k, t}$. We summarize first the results which follow from applying Proposition 4.10 and Proposition 4.11 to each $f_{t}$, in order to fix notation (note however that we shall modify slightly the lower parts of the tower maps $\hat{f}_{t}$ in Proposition 5.9): There is $\Theta_{0}>1$ so that each operator $\widehat{\mathscr{L}}_{t}$ has essential spectral radius bounded by $\Theta_{0}^{-1}$ on $\mathscr{B}_{t}=\mathscr{B}_{t}^{W_{1}^{1}}$. Outside of a disc of radius $\theta_{t}<1$ the spectrum of $\widehat{\mathscr{L}}_{t}$ on $\mathscr{B}_{t}$ consists in a simple eigenvalue at 1 , with a nonnegative eigenfunction $\hat{\phi}_{t}$, so that $\left(\hat{\phi}_{t}\right)_{0}$ belongs to $W_{1}^{2}$. Define $\Pi_{t}: \mathscr{B}_{t} \rightarrow L^{1}(I)$ by

$$
\Pi_{t}(\hat{\psi})(x)=\sum_{k \geq 0, \varsigma \in\{+,-\}} \frac{\lambda^{k}}{\left|\left(f_{t}^{k}\right)^{\prime}\left(f_{t, \varsigma}^{-k}(x)\right)\right|} \psi_{k}\left(f_{t, \varsigma}^{-k}(x)\right) \chi_{k, t}(x),
$$

where $\chi_{k, t}$ is defined like $\chi_{k}$ (see Proposition 2.7), replacing $f^{k}$ by $f_{t}^{k}$ and $c_{k}$ by $c_{k, t}$. The fixed point of the dual of $\widehat{\mathscr{L}}_{t}$ is the nonnegative measure $\nu$ on $\widehat{I}_{t}$, absolutely continuous with respect to Lebesgue on $\widehat{I}_{t}$ whose density is $w(x, k)$. If we normalize by requiring $\nu\left(\hat{\phi}_{t}\right)=1$, the invariant density of $f_{t}$ is just $\phi_{t}=\Pi_{t}\left(\hat{\phi}_{t}\right)$. Lemma 4.12 also holds for $\widehat{\mathcal{L}}_{t, M}$, using the weak norm $\|\cdot\|_{\mathscr{B}^{L^{1}}}$. This gives $\hat{\phi}_{t, M}, \nu_{t, M}=\nu_{M}$, and $\kappa_{t, M}$. If $\sigma\left(f_{t}\right), C_{1}\left(f_{t}\right)$ and $c\left(\delta, f_{t}\right)$ from Lemma 3.1 applied to $f_{t}$ are uniform in $t$, then all objects constructed are uniform in $t$ (including the $W_{1}^{2}$ norms of $\left(\hat{\phi}_{t}\right)_{0}$ and $\left(\hat{\phi}_{t, M}\right)_{0}$ for $r \in(1,2)$ ). 
There is of course some flexibility in choosing the intervals $B_{k, t}$ and the functions $\xi_{k, t}$. It is tempting, in order to get conjugated tower dynamics $\hat{f}_{t}: \hat{I}_{t} \rightarrow \hat{I}_{t}$, to choose $B_{k, t}=h_{t}\left(B_{k, 0}\right)$ and $\xi_{k, t}=\xi_{k, 0} \circ h_{t}^{-1}$, where the homeomorphisms $h_{t}$ are given by Lemma 2.11. Then, in order to prove Theorem 2.13 on linear response, one would need additional information on the $h_{t}$ (for example, but not only, the fact that $\partial_{t} h_{t}(x)=\alpha(x)$ at all points $x$ ). We shall work instead with truncated operators $\mathscr{L}_{t, M}$, disregarding the top part of the tower via Lemma 4.12, and artificially forcing the lower parts of the towers associated to the various $f_{t}$ to coincide. This is possible in view of the following consequence of Lemmas 2.10 and 2.12:

Proposition 5.9 (Controlling the truncated tower). - Let $f_{t}$ be a $C^{1}$ deformation of $S$-unimodal maps $f_{t}$ satisfying the Benedicks-Carleson condition (3) for $\mathscr{G}=14$ and $\gamma_{0}=\gamma$. Let $\hat{f}_{0}: \hat{I} \rightarrow \hat{I}$ be a tower associated to $f_{0}$ as in Section 3.1, for some $\delta>0$ and $3 \gamma_{0} / 2<\beta_{1}<\beta_{2}<2 \gamma_{0}$. Let $\alpha_{s}$ be the solution of the TCE (7) for $f_{s}$ and $v_{s}=\left.\partial_{t} f_{t}\right|_{t=s}$, with $\alpha_{s}(c)=0$, given by ${ }^{(21)}$ Theorem 2.4. Fix

$$
\frac{3 \gamma_{0}}{2}<\tilde{\beta}_{1}<\beta_{1}<\beta_{2}<\tilde{\beta}_{2}<2 \gamma_{0} .
$$

Then, for any $M \geq 1$, and for any $t$ so that

$$
\sup _{|s| \leq t}\left|\alpha_{s}\left(c_{k}\right)\right||t|<\min \left(\left(e^{-\tilde{\beta}_{1} k}-e^{-\beta_{1} k}\right),\left(e^{-\beta_{2} k}-e^{-\tilde{\beta}_{2} k}\right)\right), 1 \leq k \leq M,
$$

one can construct the tower maps $\hat{f}_{t}: \hat{I}_{t} \rightarrow \hat{I}_{t}$, the Banach spaces $\mathcal{B}_{t}^{W_{1}^{1}}, \mathcal{B}^{L^{1}}$, and the transfer operators $\widehat{\mathscr{L}}_{t}$, using parameters $\delta_{t}>0$, intervals $B_{k, t}$ admissible for $\tilde{\beta}_{1}$, and $\tilde{\beta}_{2}$, and smooth cutoff functions $\xi_{k, t}$ in such a way as to ensure

$$
\delta_{t}=\delta, \xi_{k, t}=\xi_{k}, \forall k \leq M,
$$

and, in addition, so that all results of Section 4 hold for $\widehat{\mathscr{L}}_{t}$.

If $f_{0}$ enjoys TSR then, up to taking smaller $\epsilon$, Lemma 2.12 implies that

$$
\sup _{|x| \leq \delta,|s| \leq \epsilon}\left|\alpha_{s}(x)\right||\epsilon|<\infty,
$$

so that we can exploit the above proposition.

Proof of Proposition 5.9. - Recall $h_{t}$ as given by (12) and recall Lemma 2.10. By the proof of Lemma 2.12, we have

$$
\begin{aligned}
\left|a_{k}-h_{t}\left(c_{k}\right)\right| & \leq\left|a_{k}-c_{k}\right|+\left|h_{t}\left(c_{k}\right)-h_{0}\left(c_{k}\right)\right| \\
& \leq e^{-\beta_{1} k}+\sup _{|s| \leq|t|}\left|\alpha_{s}\left(c_{k}\right)\right||t|,
\end{aligned}
$$

and

$$
\begin{aligned}
\left|a_{k}-h_{t}\left(c_{k}\right)\right| & \geq\left|a_{k}-c_{k}\right|-\left|h_{t}\left(c_{k}\right)-h_{0}\left(c_{k}\right)\right| \\
& \geq e^{-\beta_{2} k}-\sup _{|s| \leq|t|}\left|\alpha_{s}\left(c_{k}\right)\right||t| .
\end{aligned}
$$

The claim of Proposition 5.9 follows.

(21) Recall Remark 2.9. 


\section{Proof of linear response}

In this section, we prove Theorem 2.13. Let $f_{t}$ satisfy the assumptions of the theorem. We suppose in addition that the critical point is not preperiodic (the proof is much easier if it is). Applying Lemma 5.8, we fix $\epsilon>0$ and constants $\gamma, \lambda_{c}, \sigma, C_{1}, \rho, \delta$, and $c(\delta)$ and we choose $3 \gamma / 2<\beta_{1}<\beta_{2}<2 \gamma$. By Lemma 5.8, we may assume that the Benedicks-Carleson condition (3) holds for $\mathscr{G}=14$, and in some places in the proof below we may require a stronger upper bound on $\gamma$.

Constructing a tower, Banach space, and transfer operator for each $f_{t}$ as in Section 4, the invariant density of $f_{t}$ can be written as $\phi_{t}=\Pi_{t}\left(\hat{\phi}_{t}\right)$, where $\Pi_{t}$ was defined in (102), and where $\hat{\phi}_{t}$ is the nonnegative and normalized fixed point of $\widehat{\mathscr{L}}_{t}$ on $\mathscr{B}_{t}^{W_{1}^{1}}$ given by Proposition 4.11 applied to $f_{t}$. It will be convenient to work with truncated transfer operators $\widehat{\mathscr{L}}_{t, M}$, recalling Subsection 4.2, in particular Lemma 4.12, which gives $\hat{\phi}_{t, M}$. We shall in fact require the lower part of the towers of $f_{t}$ for small enough $t$, up to $M=M(t)$ as given by Proposition 5.9 to coincide with that of $f_{0}$.

When the meaning is clear, we shall remove 0 from the notation, writing, e.g., $\Pi, \hat{\phi}$, and $\hat{\phi}_{M}$, instead of $\Pi_{0}, \hat{\phi}_{0}$, and $\hat{\phi}_{0, M}$.

We start with the decomposition

$$
\phi_{t}-\phi=\Pi_{t}\left(\hat{\phi}_{t}-\hat{\phi}_{t, M}\right)+\Pi\left(\hat{\phi}_{M}-\hat{\phi}\right)+\Pi_{t}\left(\hat{\phi}_{t, M}\right)-\Pi\left(\hat{\phi}_{M}\right) .
$$

Note that if $\left.f^{k}\right|_{[c, y]}$ is injective then

$$
\int_{c_{k}}^{f^{k}(y)} \frac{\left|\psi_{k}\left(f_{+}^{-k}(z)\right)\right|}{\left|\left(f^{k}\right)^{\prime}\left(f_{+}^{-k}(z)\right)\right|} d z=\int_{c}^{y}\left|\psi_{k}(x)\right| d x .
$$

Lemma 4.12 implies that, for large enough $M$ (uniformly in $t$ )

$$
\left\|\Pi_{t}\left(\hat{\phi}_{t}-\hat{\phi}_{t, M}\right)\right\|_{L^{1}(I)} \leq C\left\|\hat{\phi}_{t}-\hat{\phi}_{t, M}\right\|_{\mathscr{B}^{L^{1}}} \leq C \tau_{M}^{\eta}, \forall|t|<\epsilon .
$$

Fix $\zeta>0$, then (106) implies

$$
\begin{array}{r}
\max \left(\left\|\Pi_{t}\left(\hat{\phi}_{t}-\hat{\phi}_{t, M}\right)\right\|_{L^{1}(I)},\left\|\Pi\left(\hat{\phi}-\hat{\phi}_{M}\right)\right\|_{L^{1}(I)}\right) \leq C|t|^{1+\zeta}, \\
\forall t \text { so that }\left(\tau_{M}^{\eta}\right)^{\frac{1}{1+\zeta}}<|t|<\epsilon .
\end{array}
$$

It is now sufficient to estimate the third term in the right-hand side of (104) for $t$ and $M=M(t)$ satisfying (107).

For this, in order to apply Proposition 5.9, and noting that the right-hand side of (103) is $\geq C e^{-2 \gamma M}$, we want $t$ and $M$ to satisfy

$$
\sup _{k,|s|<\epsilon}\left|\alpha_{s}\left(c_{k}\right)\right||t|<C e^{-2 \gamma M} .
$$

(Recall that $\sup _{k,|s|<\epsilon}\left|\alpha_{s}\left(c_{k}\right)\right| \leq L$ by Lemma 2.12.) In several places below we shall require a stronger version of (108), of the form

$$
|t|<e^{-\Gamma \gamma M}
$$

$4^{\text {e }}$ SÉRIE - TOME $45-2012-$ NNo $^{\circ}$ 
where $\Gamma>2$ is large (but uniformly bounded over the argument). Since $\tau_{M}<\lambda_{c}^{-M / 2} e^{3 M \gamma}$ (recalling Lemma 4.12) and $\lambda<e^{\gamma}$ (by (70)), we see that (107) and (109) are compatible if $\epsilon$ is small enough and

$$
\frac{1}{2} \ln \lambda_{c}>\gamma\left(\frac{1+\Gamma(1+\zeta)}{\eta}+3\right)
$$

By our TSR assumption and Lemma 5.8, we may indeed require that $\gamma$ is small enough for the above Benedicks-Carleson condition to hold, even if $\Gamma>2$ is large.

We shall call pairs $(M, t)$ so that $|t|<\epsilon$ and (107) and (109) hold admissible pairs. In the remainder of this section, $(M, t)$ will always be an admissible pair, and we shall work with the towers and operators given by Proposition 5.9 for a given such pair.

The key decomposition for an admissible pair is then

$$
\Pi_{t}\left(\hat{\phi}_{t, M}\right)-\Pi\left(\hat{\phi}_{M}\right)=\Pi_{t}\left(\hat{\phi}_{t, M}-\hat{\phi}_{M}\right)+\Pi_{t}\left(\hat{\phi}_{M}\right)-\Pi\left(\hat{\phi}_{M}\right) .
$$

Before we start with the proof, let us briefly sketch it: The term $\hat{\phi}_{t, M}-\hat{\phi}_{M}$, will be handled using spectral perturbation-type methods. This is the content of Steps 1 and 2 below, the outcome of which are claims (127), (131), and (133). (Horizontality is used here to get uniform estimates, in view of Proposition 5.9 but also, e.g., in Lemma 6.1.)

The other term requires the analysis of $\Pi_{t}-\Pi_{0}$. This will produce derivatives of the spikes, i.e., of functions of the type $\left(x-c_{k}\right)^{-1 / 2}$ (recall the definition of $\Pi_{t}$, and see Lemma 4.1). Since $\partial_{t}\left(x-h_{t}\left(c_{k}\right)\right)^{-1 / 2}$ is not integrable, this will require working with $\int A\left(\Pi_{t} \hat{\phi}_{t}-\Pi_{0} \hat{\phi}_{t}\right) d x$, with $A$ a $C^{1}$ function and integrating by parts, as well as using again horizontality. We perform this analysis in Step 3 of the proof, which yields (150).

\section{Step 1: The first term of (111): perturbation theory via resolvents}

(Recall that $(M, t)$ is an admissible pair.) In order to get a formula for the limit as $t \rightarrow 0$ (in a suitable norm) of the first term of (111) divided by $t$, we shall first analyze $\left(\hat{\phi}_{t, M}-\hat{\phi}_{M}\right) / t$, and then see how $\Pi_{t}$ enters in the picture.

Since $\nu_{M}\left(\hat{\phi}_{M}\right)=1$, we have

$$
\hat{\phi}_{t, M} \nu_{t, M}\left(\hat{\phi}_{M}\right)-\hat{\phi}_{M}=\hat{\phi}_{t, M}-\hat{\phi}_{M}+\hat{\phi}_{t, M}\left(\nu_{t, M}\left(\hat{\phi}_{M}\right)-\nu_{M}\left(\hat{\phi}_{M}\right)\right) \text {. }
$$

Now, Lemma 4.12 applied to $f$ and $f_{t}$ implies that

$$
\begin{aligned}
\left|\nu_{t, M}\left(\hat{\phi}_{M}\right)-\nu_{M}\left(\hat{\phi}_{M}\right)\right| & \leq 2 \max \left(\left\|\nu_{t, M}-\nu_{t}\right\|_{\left(\mathscr{B}^{W_{1}^{1}}\right)^{*}},\left\|\nu_{M}-\nu\right\|_{\left(\mathscr{B}^{\left.W_{1}^{1}\right) *}\right.}\right)\left\|\hat{\phi}_{M}\right\|_{\mathscr{B}^{W_{1}^{1}}} \\
& \leq C \tau_{M}^{\eta}\left\|\hat{\phi}_{M}\right\|_{\mathscr{B}_{1}^{W_{1}^{1}}} .
\end{aligned}
$$

Our choices imply that $C \tau_{M}^{\eta}=O\left(|t|^{1+\zeta}\right)$ while $\left\|\hat{\phi}_{M}\right\|_{\mathscr{B}^{W_{1}^{1}}}$ is uniformly bounded, e.g., by the proof of Lemma 4.12. Therefore, to study $\hat{\phi}_{t, M}-\hat{\phi}_{M}$, it suffices to estimate $\hat{\phi}_{t, M} \nu_{t, M}\left(\hat{\phi}_{M}\right)-\hat{\phi}_{M}$, that we shall express as a difference of spectral projectors.

Next, set

$$
\widehat{Q}_{t, M}=\widehat{Q}_{t, M}(z)=z-\widehat{\mathscr{L}}_{t, M}, \quad \widehat{Q}_{M}=\widehat{Q}_{M}(z)=z-\widehat{\mathscr{L}}_{M},
$$

recall $\mathbb{P}_{M}$ from the proof of Lemma 4.12, and denote by

$$
\mathbb{P}_{t, M}(\hat{\psi})=\hat{\phi}_{t, M} \nu_{t, M}(\hat{\psi})
$$


the spectral projector corresponding to the maximal eigenvalue of $\widehat{\mathscr{L}}_{t, M}$. Using

$$
\widehat{Q}_{t, M}^{-1}-\widehat{Q}_{M}^{-1}=\widehat{Q}_{t, M}^{-1}\left(\widehat{\mathscr{L}}_{t, M}-\widehat{\mathscr{L}}_{M}\right) \widehat{Q}_{M}^{-1}, \text { and } Q_{M}^{-1}\left(\hat{\phi}_{M}\right)=\frac{\hat{\phi}_{M}}{z-\kappa_{M}},
$$

we rewrite $\hat{\phi}_{t, M} \nu_{t, M}\left(\hat{\phi}_{M}\right)-\hat{\phi}_{M}=\left(\mathbb{P}_{t, M}-\mathbb{P}_{M}\right)\left(\hat{\phi}_{M}\right)$ as follows:

$$
\begin{aligned}
\hat{\phi}_{t, M} \nu_{t, M}\left(\hat{\phi}_{M}\right)-\hat{\phi}_{M} & =-\frac{1}{2 i \pi} \oint \frac{\widehat{\mathscr{Q}}_{t, M}^{-1}(z)}{z-\kappa_{M}}\left(\widehat{\mathscr{L}}_{t, M}-\widehat{\mathscr{L}}_{M}\right)\left(\hat{\phi}_{M}\right) d z \\
& =\left(\kappa_{M}-\widehat{\mathscr{L}}_{t, M}\right)^{-1}\left(\mathrm{id}-\mathbb{P}_{t, M}\right)\left(\widehat{\mathscr{L}}_{t, M}-\widehat{\mathscr{L}}_{M}\right)\left(\hat{\phi}_{M}\right),
\end{aligned}
$$

where the contour is a circle centered at 1 , outside of the disc of radius $\max \left(\theta_{0}, \theta_{t}\right)$ (using the notation from Subsection 5.2).

We are going to use again the arguments in [29]. By uniformity of the constants in Lemma 5.8, the Lasota-Yorke estimates ${ }^{(22)}$ in the proofs of Proposition 4.10 and Lemma 4.12 say that there exist $\epsilon>0$ and $C \geq 1$ so that, for all $|t| \leq \epsilon$, all $M$, all $j$,

$$
\begin{aligned}
& \left\|\hat{\mathscr{L}}_{t, M}^{j}(\hat{\psi})\right\|_{\mathcal{B}^{L^{1}}} \leq C\|\hat{\psi}\|_{\mathcal{B}^{L^{1}}}, \quad\left\|\hat{\mathscr{L}}_{t, M}^{j}(\hat{\psi})\right\|_{\mathscr{B}^{W_{1}^{1}}} \leq C \Theta^{-j}\|\hat{\psi}\|_{\mathscr{B}^{W_{1}^{1}}}+C\|\hat{\psi}\|_{\mathcal{B}^{L^{1}}}, \\
& \left\|\widehat{\mathscr{L}}_{M}^{j}(\hat{\psi})\right\|_{\mathcal{B}^{L^{1}}} \leq C\|\hat{\psi}\|_{\mathscr{B}^{L^{1}}}, \quad\left\|\widehat{\mathscr{L}}_{M}^{j}(\hat{\psi})\right\|_{\mathscr{B}^{W_{1}^{1}}} \leq C \Theta^{-j}\|\hat{\psi}\|_{\mathcal{B}^{W_{1}^{1}}}+C\|\hat{\psi}\|_{\mathcal{B}^{L^{1}}} .
\end{aligned}
$$

In Step 2 we shall find $\widetilde{C} \geq 1$ and $\tilde{\eta}>0$ so that for each admissible pair $(M, t)$

$$
\left\|\widehat{\mathscr{L}}_{t, M}(\hat{\psi})-\widehat{\mathscr{L}}_{M}(\hat{\psi})\right\|_{\mathcal{B}^{L^{1}}} \leq \widetilde{C}|t|^{\tilde{\eta}}\|\hat{\psi}\|_{\mathcal{B}}, \forall \hat{\psi} \in \mathcal{B}
$$

We are not exactly in the setting of [29], since we have a "moving target" $\widehat{\mathscr{L}}_{M(t)}$ as $t \rightarrow 0$. However, since the right-hand side of (115) does not depend on $M$, setting

$$
\mathcal{N}_{t, M}:=\left(\kappa_{M}-\widehat{\mathscr{L}}_{t, M}\right)^{-1}\left(\mathrm{id}-\mathbb{P}_{t, M}\right)-\left(\kappa_{M}-\widehat{\mathscr{L}}_{M}\right)^{-1}\left(\mathrm{id}-\mathbb{P}_{M}\right),
$$

then (113)-(114) and (115) imply, by a small modification of the proofs of [29, Theorem 1, Corollary 1], that there exist $\widehat{C} \geq 1$ and $\hat{\eta}>0$ so that for all admissible pairs $(M, t)$

$$
\left\|\mathcal{N}_{t, M}\right\|_{\mathscr{B}^{W_{1}^{1}}} \leq \widehat{C}, \quad\left\|\mathcal{N}_{t, M}(\hat{\psi})\right\|_{\mathscr{B}^{L^{1}}} \leq \widehat{C}|t|^{\tilde{\eta} \hat{\eta}}\|\hat{\psi}\|_{\mathscr{B}^{W_{1}^{1}}}
$$

In Step 2, we shall show that there exist $C>0$ and $\mathscr{D}_{M} \in \mathscr{B}=\mathscr{B}^{W_{1}^{1}}$ with

$$
\int_{I} \mathscr{D}_{M, 0} d x=0, \mathscr{D}_{M, k}=0, \forall k \geq 1, \text { and }\left\|\mathscr{D}_{M}\right\|_{\mathscr{B}} \leq C,
$$

and $\tilde{\zeta}>0$, so that for all admissible pairs $(M, t)$

$$
\left\|\widehat{\mathscr{L}}_{t, M}\left(\hat{\phi}_{M}\right)-\widehat{\mathscr{L}}_{M}\left(\hat{\phi}_{M}\right)-t \mathscr{D}_{M}\right\|_{\mathcal{B}} \leq C|t|^{1+\tilde{\zeta}} .
$$

Writing

$$
\left(\kappa_{M}-\widehat{\mathscr{L}}_{t, M}\right)^{-1}\left(\mathrm{id}-\mathbb{P}_{t, M}\right)=\mathcal{N}_{t, M}+\left(\kappa_{M}-\widehat{\mathscr{L}}_{M}\right)^{-1}\left(\mathrm{id}-\mathbb{P}_{M}\right),
$$

we see that (117) together with (125) and (112) imply

$$
\begin{aligned}
\hat{\phi}_{t, M}-\hat{\phi}_{M} & =\left[\mathcal{N}_{t, M}+\left(\kappa_{M}-\widehat{\mathscr{L}}_{M}\right)^{-1}\left(\mathrm{id}-\mathbb{P}_{M}\right)\right]\left(t \mathscr{D}_{M}+O_{\mathscr{B}}\left(|t|^{1+\tilde{\zeta}}\right)\right) \\
& =t \mathcal{N}_{t, M}\left(\mathscr{D}_{M}\right)+t\left(\kappa_{M}-\widehat{\mathscr{L}}_{M}\right)^{-1}\left(\mathrm{id}-\mathbb{P}_{M}\right)\left(\mathscr{D}_{M}\right)+\Delta O_{\mathscr{B}_{1}^{1}}\left(|t|^{1+\tilde{\zeta}}\right)
\end{aligned}
$$

(22) We use that the constant $c_{f_{t}}(\delta)$ associated to $f_{t}$ by Lemma 5.8 does not depend on $t$ and that $\inf _{\delta \geq \delta_{0}} c(\delta)>0$ for any $\delta_{0}>0$. 
Note for further use (in (130) below) that, since $\|\hat{\psi}\|_{C^{0}}:=\sup _{k} \sup \left|\psi_{k}\right| \leq C\|\hat{\psi}\|_{W_{1}^{1}}$, the bound (118) with the first inequality in (116) imply

$$
\left\|\hat{\phi}_{t, M}-\hat{\phi}_{M}\right\|_{C^{0}}=O(t), \text { as } t \rightarrow 0 \text {, uniformly in admissible pairs }(M, t) .
$$

Recalling from (18) the definition of $\hat{Y}$, we shall see in Step 2 that the following expression defines an element of $\mathscr{B}^{W_{1}^{1}}$

$$
\mathscr{D}=-\left(\mathcal{J}_{0}(\widehat{\mathscr{L}}(\hat{Y} \hat{\phi}))^{\prime}\right.
$$

and that, in addition $\lim _{M \rightarrow \infty}\left\|\mathscr{D}-\mathscr{D}_{M}\right\|_{W_{1}^{1}}=0$. More precisely, there is $\zeta^{\prime}>0$ so that for admissible pairs $(M, t) \rightarrow(\infty, 0)$

$$
\left\|\mathscr{D}-\mathscr{D}_{M}\right\|_{W_{1}^{1}}=\left\|\mathscr{D}_{0}-\mathscr{D}_{M, 0}\right\|_{W_{1}^{1}(I)}=O\left(|t|^{\zeta^{\prime}}\right) .
$$

Note that $\mathscr{D}_{k}=0$ for $k \geq 1$, and that $\int \mathscr{D}_{0} d x=0$, so that $\nu(\mathscr{D})=0$. Similarly, $\nu\left(\mathscr{D}_{M}\right)=0$. Therefore, by the spectral properties of $\widehat{\mathscr{L}}$ on $\mathscr{B}^{W_{1}^{1}}$ from Propositions 4.10 and 4 .11, we have that

$$
(\mathrm{id}-\widehat{\mathscr{L}})^{-1}(\mathscr{D}) \in \mathscr{B}^{W_{1}^{1}}, \quad(\mathrm{id}-\widehat{\mathscr{L}})^{-1}\left(\mathscr{D}_{M}\right) \in \mathscr{B}^{W_{1}^{1}}
$$

Now, by the Lasota-Yorke estimates in the beginning of the proof of Lemma 4.12, the first claim of [29, Theorem 1] gives an integer $M_{0}$ and a small disc $B$ around 1 so that

$$
\sup _{M \geq M_{0}} \sup _{z \notin B}\left\|z-\widehat{\mathscr{L}}_{M}\right\|_{\mathscr{B}^{W_{1}^{1}}}^{-1}<\infty
$$

while, letting $\mathbb{P}_{M}(\hat{\psi})=\hat{\phi}_{M} \nu_{M}(\hat{\psi})$ be the spectral projector corresponding to the maximal eigenvalue of $\widehat{\mathscr{L}}_{M}$, and setting

$$
\mathcal{N}_{M}:=\left(\kappa_{M}-\widehat{\mathscr{L}}_{M}\right)^{-1}\left(\mathrm{id}-\mathbb{P}_{M}\right)-\left(\mathrm{id}-\widehat{\mathscr{L}}_{0}\right)^{-1}(\mathrm{id}-\hat{\phi} \nu(\cdot)),
$$

the second claim of [29, Theorem 1] with the first lines of [10, Appendix B] give

$$
\left\|\mathcal{N}_{M}(\hat{\psi})\right\|_{\mathscr{B}^{L^{1}}} \leq \widehat{C} \tau_{M}^{\eta}\|\hat{\psi}\|_{\mathscr{B}^{W_{1}^{1}}}
$$

and

$$
\Delta:=\left\|\left(\kappa_{M}-\widehat{\mathscr{L}}_{M}\right)^{-1}\left(\mathrm{id}-\mathbb{P}_{M}\right)\right\|_{\mathscr{B}^{W_{1}^{1}}}<\infty .
$$

The estimate (124) and our condition (107) on $M$ then give

$$
\left\|\mathcal{N}_{M}(\hat{\psi})\right\|_{\mathscr{B}^{L^{1}}} \leq \widehat{C}|t|^{1+\zeta}\|\hat{\psi}\|_{\mathscr{B}^{W_{1}^{1}}}
$$

In particular, since $\left\|\mathscr{D}_{M}\right\|_{\mathscr{B}} \leq C$, we get $\lim _{t \rightarrow 0}\left\|\mathcal{N}_{M}\left(\mathscr{D}_{M}\right)\right\|_{\mathscr{B}^{L^{1}}}=0$, exponentially in $M$. Therefore, recalling that $\nu(\mathscr{D})=\nu\left(\mathscr{D}_{M}\right)=0$, and using (121), there exists $\zeta^{\prime \prime}>0$ so that for admissible $(M, t) \rightarrow(\infty, 0)$,

$$
\begin{aligned}
& \left\|\left(\kappa_{M}-\widehat{\mathscr{L}}_{M}\right)^{-1}\left(\mathrm{id}-\mathbb{P}_{M}\right)\left(\mathscr{D}_{M}\right)-(\mathrm{id}-\widehat{\mathscr{L}})^{-1}(\mathscr{D})\right\|_{\mathcal{B}^{L^{1}}} \\
& \quad \leq\left\|\mathcal{N}_{M}\left(\mathscr{D}_{M}\right)\right\|_{\mathcal{B}^{L^{1}}}+\left\|(\mathrm{id}-\widehat{\mathscr{L}})^{-1}\left(\mathscr{D}-\mathscr{D}_{M}\right)\right\|_{\mathcal{B}_{1}^{W_{1}^{1}}}=O\left(|t|^{\zeta^{\prime \prime}}\right) .
\end{aligned}
$$

We may now conclude the first part of Step 1: Dividing (118) by $t$, letting $t \rightarrow 0$, and applying the second bound of (116) gives $\hat{\zeta}>0$ so that (using again $\nu(\mathscr{D})=0$ )

$$
\left\|\frac{1}{t}\left(\hat{\phi}_{t, M(t)}-\hat{\phi}_{M(t)}\right)-(\mathrm{id}-\widehat{\mathscr{L}})^{-1}(\mathscr{D})\right\|_{\mathscr{B}^{L^{1}}}=O\left(|t|^{\hat{\zeta}}\right) \text { as } t \rightarrow 0 .
$$


It remains to assess the effect of composition by $\Pi_{t}$ in the first term of (111) divided by $t$. We claim that (127) implies

$$
\lim _{t \rightarrow 0}\left\|\frac{1}{t} \Pi_{t}\left[\hat{\phi}_{t, M}-\hat{\phi}_{M}\right]-\Pi_{0}\left((\mathrm{id}-\widehat{\mathcal{L}})^{-1}(\mathscr{D})\right)\right\|_{L^{1}(I)}=0 .
$$

To prove (128), we start from the decomposition

$$
\frac{1}{t} \Pi_{t}\left(\hat{\phi}_{t, M}-\hat{\phi}_{M}\right)=\frac{1}{t} \Pi_{0}\left(\hat{\phi}_{t, M}-\hat{\phi}_{M}\right)+\frac{1}{t}\left(\Pi_{t}-\Pi_{0}\right)\left(\hat{\phi}_{t, M}-\hat{\phi}_{M}\right) .
$$

Note that (105) implies $\left\|\Pi_{0} \mathcal{T}_{M}(\hat{\psi})\right\|_{L^{1}} \leq C\|\hat{\psi}\|_{\mathscr{B}^{L^{1}}}$. Therefore, since $\nu(\mathscr{D})=0,(127)$ takes care of the first term in (129), and it suffices to show that the second term in (129) tends to zero in $L^{1}(I)$ as $t \rightarrow 0$.

Recall that Lemma 5.8 allows us to take a larger value of $\Gamma$ in (109) if necessary. Estimate (141) in Step 3 implies

$$
\left\|\left(\Pi_{t}-\Pi_{0}\right)(\hat{\psi})\right\|_{L^{1}(I)} \leq C \sqrt{t}\|\hat{\psi}\|_{C^{0}} .
$$

Therefore, by (119), we have

$$
\left\|\left(\Pi_{t}-\Pi_{0}\right)\left(\hat{\phi}_{t, M}-\hat{\phi}_{M}\right)\right\|_{L^{1}} \leq C \sqrt{t}\left\|\hat{\phi}_{t, M}-\hat{\phi}_{M}\right\|_{C^{0}}=o(t),
$$

proving (128).

Finally, (128) immediately implies that

$$
\lim _{t \rightarrow 0} \frac{1}{t} \int A \Pi_{t}\left[\hat{\phi}_{t, M}-\hat{\phi}_{M}\right] d x=-\int A \Pi_{0}\left((\mathrm{id}-\widehat{\mathscr{L}})^{-1}\left[\mathcal{T}_{0}(\widehat{\mathscr{L}}(\hat{Y} \hat{\phi}))^{\prime}\right]\right) d x .
$$

In other words,

$$
\lim _{t \rightarrow 0} \frac{1}{t} \int(A-A \circ f) \Pi_{t}\left[\hat{\phi}_{t, M}-\hat{\phi}_{M}\right] d x=-\int A\left(\mathcal{J}_{0}(\widehat{\mathscr{L}}(\hat{Y} \hat{\phi}))\right)^{\prime} d x
$$

where we used (78). If $A$ is $C^{1}$, we can integrate by parts, and we find

$$
\lim _{t \rightarrow 0} \frac{1}{t} \int(A-A \circ f) \Pi_{t}\left[\hat{\phi}_{t, M}-\hat{\phi}_{M}\right] d x=\int A^{\prime} \mathcal{T}_{0}(\widehat{\mathscr{L}}(\hat{Y} \hat{\phi})) d x .
$$

Step 2: The first term of (111): Computing $\lim \frac{1}{t}\left(\widehat{\mathscr{L}}_{t, M}-\widehat{\mathscr{L}}_{M}\right)\left(\hat{\phi}_{M}\right)$.

In this step, we prove (115), (117), and (120), (121), for admissible pairs $(M, t)$. The following estimates will play a crucial part in the argument (their proof is given in Appendix $C$, it uses the fact that $t \mapsto f_{t} \in C^{3}$ is a $C^{2}$ map):

Lemma 6.1 (Taylor series for $f_{ \pm}^{-k}(x)-f_{t, \pm}^{-k}(x)$ ). - Let $f_{t}$ satisfy the assumptions of Theorem 2.13. Recall the functions $Y_{k, t}$ from (17), the maps $f_{t, \pm}^{-k}$ from (1), and the smooth cutoff functions $\xi_{k}$ from Definition 4.7. Then there is $C>0$ so that for any $k \geq H_{0}$ and any $|s| \leq \epsilon$, if (108) holds, then

$$
\sup _{y \in \widetilde{I}_{k}} \frac{\left|Y_{k, s}(y)\right|}{\left|\left(f_{s}^{k}\right)^{\prime}(y)\right|} \leq C \frac{e^{2 \gamma k}}{\left|\left(f_{s}^{k-1}\right)^{\prime}\left(c_{1, s}\right)\right|^{1 / 2}} .
$$

$4^{\mathrm{e}}$ SÉRIE - TOME $45-2012-\mathrm{N}^{\circ} 6$ 
In addition, for all $k \geq 1$, we have

$$
\begin{aligned}
& \sup _{x \in f_{s}^{k}\left(\widetilde{I}_{k}\right)}\left|\partial_{x}\left(\frac{Y_{k, s}\left(f_{s, \pm}^{-k}(x)\right)}{\left(f_{s}^{k}\right)^{\prime}\left(f_{s, \pm}^{-k}(x)\right)}\right)\right| \leq C \frac{e^{3 \gamma k}}{\left|\left(f_{s}^{k-1}\right)^{\prime}\left(c_{1, s}\right)\right|^{1 / 2}}, \\
& \sup _{x \in f_{s}^{k}\left(\widetilde{I}_{k}\right)}\left|\partial_{x}^{2}\left(\frac{Y_{k, s}\left(f_{s, \pm}^{-k}(x)\right)}{\left(f_{s}^{k}\right)^{\prime}\left(f_{s, \pm}^{-k}(x)\right)}\right)\right| \leq C \frac{e^{6 \gamma k}}{\left|\left(f_{s}^{k-1}\right)^{\prime}\left(c_{1, s}\right)\right|^{1 / 2}} .
\end{aligned}
$$

Finally, for all $k \leq M$ and all $x \in f_{s}^{k}\left(\widetilde{I}_{k}\right)$

$$
\left|f_{ \pm}^{-k}(x)-f_{t, \pm}^{-k}(x)-t \frac{Y_{k}\left(f_{ \pm}^{-k}(x)\right)}{\left(f^{k}\right)^{\prime}\left(f_{ \pm}^{-k}(x)\right)}\right| \leq C|t|^{2} e^{4 \gamma k}
$$

and, for the same $k$ and $x$

$$
\left|\frac{1}{\left(f_{t}^{k}\right)^{\prime}\left(f_{ \pm}^{-k}(x)\right)}-\frac{1}{\left(f^{k}\right)^{\prime}\left(f_{t, \pm}^{-k}(x)\right)}-t\left(\frac{Y_{k}\left(f_{ \pm}^{-k}(x)\right)}{\left(f^{k}\right)^{\prime}\left(f_{ \pm}^{-k}(x)\right)}\right)^{\prime}\right| \leq C|t|^{2} e^{7 \gamma k}
$$

We first prove (115). If $j>M$ then $\widehat{\mathscr{L}}_{t, M}(\hat{\psi})(x, j)=\widehat{\mathscr{L}}_{M}(\hat{\psi})(x, j)=0$. If $1 \leq j \leq M$, since $\xi_{j}=\xi_{j, t}$ (recall the construction in Proposition 5.9),

$$
\widehat{\mathscr{L}}_{t, M}(\hat{\psi})(x, j)-\widehat{\mathscr{L}}_{M}(\hat{\psi})(x, j)=0 .
$$

Therefore, we need only worry about $j=0$.

Recall the Definition (76) of $\widehat{\mathscr{L}}_{t, M}(\hat{\psi})(x, 0)$. The Definition (18) of $\hat{Y}_{s}$ (the shift in indices there mirrors that in (76)) together with (162) and (165) from the proof of Lemma 6.1 imply the following: Assume that $\varphi$ is $C^{1}$ and supported in $\widetilde{I}_{k}$. Then there exists $s(t) \in[0, t]$ so that

$$
\begin{aligned}
& \left|\frac{\varphi\left(f_{+}^{-k}(x)\right)}{\left|\left(f^{k}\right)^{\prime}\left(f_{+}^{-k}(x)\right)\right|}-\frac{\varphi\left(f_{t,+}^{-k}(x)\right)}{\left|\left(f_{t}^{k}\right)^{\prime}\left(f_{t,+}^{-k}(x)\right)\right|}\right| \\
& \leq|t|\left|\varphi\left(f_{s,+}^{-k}(x)\right)\right|\left|\left(\frac{Y_{k, s}\left(f_{s,+}^{-k}(x)\right)}{\left(f_{s}^{k}\right)^{\prime}\left(f_{s,+}^{-k}(x)\right)^{\prime}}\right)^{\prime}\right|+|t| \frac{\left|\varphi^{\prime}\left(f_{s,+}^{-k}(x)\right)\right|}{\left|\left(f_{s}^{k}\right)^{\prime}\left(f_{s,+}^{-k}(x)\right)\right|}\left|\frac{Y_{k, s}\left(f_{s,+}^{-k}(x)\right)}{\left(f_{s}^{k}\right)^{\prime}\left(f_{s,+}^{-k}(x)\right)}\right| .
\end{aligned}
$$

Of course, the branch $f_{-}^{-k}$ is handled similarly. Recall that $C^{\infty}$ is dense in $W_{1}^{1}$. Therefore, summing over the inverse branches, and taking into account the contribution of $\left(1-\xi_{k}\right)\left(f_{t,+}^{-k}(x)\right)-\left(1-\xi_{k}\right)\left(f_{+}^{-k}(x)\right)$ via (75) or (81) (our assumptions imply that each $\psi_{k}^{\prime}$ and $\xi_{k}^{\prime}$ vanishes at the boundary of its support), and averaging, we get $C>0$ so that for any $\hat{\psi} \in \mathscr{B}$ and any admissible pair $(M, t)$, using (134) and (135) from Lemma 6.1 and the upper bound (70) on $\lambda$

$$
\left\|\widehat{\mathscr{L}}_{t, M}(\hat{\psi})-\widehat{\mathscr{L}}_{M}(\hat{\psi})\right\|_{\mathcal{B}^{L^{1}}} \leq C|t| e^{5 \gamma M} \lambda^{2 M}\|\hat{\psi}\|_{\mathscr{B}^{W_{1}^{1}}} \leq C|t| e^{7 \gamma M}\|\hat{\psi}\|_{\mathcal{B}^{W_{1}^{1}}} .
$$

In view of (109), this proves (115).

Next, we show (117). Note that

$$
\begin{aligned}
\varphi\left(f_{+}^{-k}(x)\right)\left(\frac{Y_{k}\left(f_{+}^{-k}(x)\right)}{\left(f^{k}\right)^{\prime}\left(f_{+}^{-k}(x)\right)}\right)^{\prime}+\frac{\varphi^{\prime}\left(f_{+}^{-k}(x)\right)}{\left(f^{k}\right)^{\prime}\left(f_{+}^{-k}(x)\right)} \frac{Y_{k}\left(f_{+}^{-k}(x)\right)}{\left(f^{k}\right)^{\prime}\left(f_{+}^{-k}(x)\right)} \\
=\left(\frac{\varphi\left(f_{+}^{-k}(x)\right) Y_{k}\left(f_{+}^{-k}(x)\right)}{\left(f^{k}\right)^{\prime}\left(f_{+}^{-k}(x)\right)}\right)^{\prime},
\end{aligned}
$$


and set (recall that $\left.\left(\hat{\phi}_{M}\right)_{0}^{\prime} \in W_{1}^{1}\right)$

$$
\mathscr{D}_{M}:=-\left(\mathcal{J}_{0}\left(\widehat{\mathscr{L}}_{M}\left(\hat{Y} \hat{\phi}_{M}\right)\right)\right)^{\prime} \in \mathscr{B} \text {. }
$$

Clearly $\nu\left(\mathscr{D}_{M}\right)=0$, integrating by parts. Since Lemma 4.12 implies that $\hat{\phi}_{M}$ is an eigenvector of $\widehat{\mathscr{L}}_{M}$ for an eigenvalue $\kappa_{M}$ close to 1 (so that the $\lambda^{k}$ factor can be replaced by $\kappa_{M}^{k}$, which is strictly smaller than $C e^{2 \gamma k}$ by our choices), we get, using (134) and (135) from Lemma 6.1, as well as Lemma 4.1, $\sup _{M}\left\|\mathscr{D}_{M}\right\|_{\mathscr{B}^{L^{1}}}<\infty$. Up to increasing $\Gamma$ in (109), (135) and (136) imply that $\sup _{M}\left\|\mathscr{D}_{M}\right\|_{\mathscr{B}_{1}^{1}}<\infty$.

Using again that $\left(\hat{\phi}_{M}\right)_{0}^{\prime} \in W_{1}^{1}$, we may write the average of the $t$-Taylor series of order two of

$$
\frac{\hat{\phi}_{M, k-1}\left(f_{+}^{-k}(x)\right)}{\left|\left(f^{k}\right)^{\prime}\left(f_{+}^{-k}(x)\right)\right|}-\frac{\hat{\phi}_{M, k-1}\left(f_{t,+}^{-k}(x)\right)}{\left|\left(f_{t}^{k}\right)^{\prime}\left(f_{t,+}^{-k}(x)\right)\right|},
$$

and of its $x$-derivative. By (136), (137), and (138), this gives

$$
\left\|\widehat{\mathscr{L}}_{t, M}\left(\hat{\phi}_{M}\right)-\widehat{\mathscr{L}}_{M}\left(\hat{\phi}_{M}\right)-t \mathscr{D}_{M}\right\|_{\mathcal{B}} \leq C e^{11 \gamma M}|t|^{1+\zeta} .
$$

Since we can take $\Gamma$ in (109) as large as necessary, this establishes (117).

Set $\mathscr{D}=-\mathcal{T}_{0}(\widehat{\mathscr{L}}(\hat{Y} \hat{\phi}))^{\prime}$. Clearly, $\nu(\mathscr{D})=0$, integrating by parts. The estimates we proved imply that $\left\|\mathscr{D}_{0}-\mathscr{D}_{M, 0}\right\|_{W_{1}^{1}} \rightarrow 0$, exponentially fast as $M \rightarrow \infty$, and that $\mathscr{D} \in \mathscr{B}^{W_{1}^{1}}$. This shows (120) and (121).

\section{Step 3: The second term of (111): Estimating $\frac{1}{t}\left(\Pi_{t}-\Pi_{0}\right)\left(\hat{\phi}_{M}\right) \in\left(C^{1}(I)\right)^{*}$}

In this step, the points are not necessarily falling from the tower, so that the analogues of the derivatives in Lemma 6.1 have nonintegrable spikes. Therefore, as already mentioned, we shall not only require horizontality, but we shall also need to perform integration by parts, using that the observable $A$ is $C^{1}$.

As before, the index $k$ ranges between 1 and $M$, where $(M, t)$ is an admissible pair. We focus on the branch $f_{+}^{-k}$, the other one is handled in a similar way.

Note for further use that, recalling Proposition 5.9, Lemma 4.1 implies that there exists $C$ so that for any admissible pair $(M, t)$ and any $1 \leq k \leq M$

$$
\Psi_{k, t}:=\sup _{z \in\left[c_{k}, c_{k, t}\right]} \int_{c_{k}}^{z} \frac{\lambda^{k}}{\left|\left(f^{k}\right)^{\prime}\left(f_{+}^{-k}(x)\right)\right|} d x \leq \int_{c_{k}}^{c_{k, t}} \frac{C}{\sqrt{x-c_{k}}} d x \leq C L|t|^{1 / 2} .
$$

(We used (14) to get $\left|c_{k, t}-c_{k}\right|=\left|h_{t}\left(c_{k}\right)-h_{0}\left(c_{k}\right)\right| \leq L|t|$.) In particular,

$$
\left|f_{+}^{-k}\left(c_{k, t}\right)-f_{+}^{-k}\left(c_{k}\right)\right| \leq \Psi_{k, t} \leq C|t|^{1 / 2} .
$$

Assume to fix ideas that $c_{k}>c_{k, t}$, with $c_{k}$ and $c_{k, t}$ local maxima for $f^{k}$ and $f_{t}^{k}$, respectively (the other possibilities are treated similarly and left to the reader). 
We first study the points in $f^{k}\left(\operatorname{supp}\left(\phi_{M, k}\right)\right)$ for which $f_{+}^{-k}(x)$ exists but not $f_{t,+}^{-k}(x)$, i.e., the interval $\left[c_{k, t}, c_{k}\right]$. This gives the following contribution:

$$
\begin{aligned}
-\int_{c_{k, t}}^{c_{k}} A(x) & \frac{\lambda^{k}}{\left|\left(f^{k}\right)^{\prime}\left(f_{+}^{-k}(x)\right)\right|} \phi_{M, k}\left(f_{+}^{-k}(x)\right) d x \\
& =\int_{f_{+}^{-k}\left(c_{k, t}\right)}^{c_{0}}\left[\left(A\left(f^{k}(y)\right)-A\left(c_{k}\right)\right)+A\left(c_{k}\right)\right] \lambda^{k} \phi_{M, k}(y) d y \\
& =\int_{f_{+}^{-k}\left(c_{k, t}\right)}^{c_{0}} A^{\prime}\left(z_{k, t}(y)\right)\left(c_{k}-f^{k}(y)\right) \lambda^{k} \phi_{M, k}(y) d y \\
& \quad A\left(c_{k}\right) \int_{f_{+}^{-k}\left(c_{k, t}\right)}^{c_{0}} \lambda^{k} \phi_{M, k}(y) d y
\end{aligned}
$$

where $z_{k, t}(y) \in\left[c_{k, t}, c_{k}\right]$, and we used that $f_{+}^{-k}$ is orientation reversing. Now

$$
\begin{aligned}
& \left|\int_{f_{+}^{-k}\left(c_{k, t}\right)}^{c_{0}} A^{\prime}\left(z_{k, t}(y)\right)\left(c_{k}-f^{k}(y)\right) \lambda^{k} \phi_{M, k}(y) d y\right| \\
& \quad \leq \sup \left|A^{\prime}\right|\left|c_{k}-c_{k, t}\right| \int_{c_{k, t}}^{c_{k}} \frac{\lambda^{k}}{\left|\left(f^{k}\right)^{\prime}\left(f_{+}^{-k}(x)\right)\right|} \phi_{M, k}\left(f_{+}^{-k}(x)\right) d x \\
& \quad \leq C \sup \left|A^{\prime}\right|\left|c_{k}-c_{k, t}\right| \sqrt{c_{k}-c_{k, t}} \\
& \quad \leq C \sup \left|A^{\prime}\right| \sup _{s}\left|\alpha_{s}\left(c_{k}\right)\right|^{3 / 2}|t|^{3 / 2}
\end{aligned}
$$

where we used Lemma 2.12 together with (141). Since there are $M$ terms and since $\lim _{t \rightarrow 0} M \sqrt{|t|}=0$ for admissible pairs $(M, t)$, the relevant contribution of (143) is fully contained in the last line (144) of (143). (We shall see in a moment that (144) cancels out exactly with another term.)

Second, we need to consider

$$
\begin{gathered}
-\int_{-1}^{c_{k, t}} \lambda^{k} A(x)\left(\frac{\phi_{M, k}\left(f_{+}^{-k}(x)\right)}{\left|\left(f^{k}\right)^{\prime}\left(f_{+}^{-k}(x)\right)\right|}-\frac{\phi_{M, k}\left(f_{t,+}^{-k}(x)\right)}{\left|\left(f_{t}^{k}\right)^{\prime}\left(f_{t,+}^{-k}(x)\right)\right|}\right) d x \\
=-\int_{-1}^{c_{k, t}} \lambda^{k} A^{\prime}(x)\left[\tilde{\phi}_{M, k}\left(f_{+}^{-k}(x)\right)-\tilde{\phi}_{M, k}\left(f_{t,+}^{-k}(x)\right)\right] d x \\
\quad+\left.\lambda^{k}\left[A(x)\left(\tilde{\phi}_{M, k}\left(f_{+}^{-k}(x)\right)-\tilde{\phi}_{M, k}\left(f_{t,+}^{-k}(x)\right)\right)\right]\right|_{-1} ^{c_{k, t}},
\end{gathered}
$$

where $\tilde{\phi}_{M, k}^{\prime}=\phi_{M, k}, \tilde{\phi}_{M, k}(-1)=0$, and we used that $\left(f^{k}\right)^{\prime}\left(f_{+}^{-k}(x)\right)<0$ when integrating by parts. One term in (147) vanishes because of the support of $\tilde{\phi}_{M, k}$. The other term is

$$
\lambda^{k} A\left(c_{k, t}\right)\left(\tilde{\phi}_{M, k}\left(f_{+}^{-k}\left(c_{k, t}\right)\right)-\tilde{\phi}_{M, k}\left(f_{t,+}^{-k}\left(c_{k, t}\right)\right)\right) .
$$

Since $A\left(c_{k, t}\right)=A\left(c_{k}\right)+A^{\prime}\left(z_{k, t}\right)\left(c_{k, t}-c_{k}\right)$ for $z_{k, t} \in\left[c_{k, t}, c_{k}\right]$, and since $f_{t,+}^{-k}\left(c_{k, t}\right)=c_{0}$, (148) can be written as

$$
\lambda^{k} A\left(c_{k}\right)\left(\tilde{\phi}_{M, k}\left(f_{+}^{-k}\left(c_{k, t}\right)\right)-\tilde{\phi}_{M, k}\left(c_{0}\right)\right)+H_{k, t},
$$

with $\left|H_{k, t}\right| \leq C|t|^{3 / 2}$, uniformly in $k \leq M$ for admissible pairs $(M, t)$ (recall (14) and (142)). Summing over $1 \leq k \leq M$ and dividing by $t$, we have proved that, as $t \rightarrow 0$, the contributions from (148) cancel out exactly with the singular terms from line (144). (Recall that $(M, t)$ are admissible, in particular (109) holds.) 
The other term, (146), is

$$
\begin{aligned}
& -\int_{-1}^{c_{k, t}} \lambda^{k} A^{\prime}(x)\left[\tilde{\phi}_{M, k}\left(f_{+}^{-k}(x)\right)-\tilde{\phi}_{M, k}\left(f_{t,+}^{-k}(x)\right)\right] d x \\
& \quad=-\int_{-1}^{c_{k, t}} \lambda^{k} A^{\prime}(x) \phi_{M, k}\left(f_{u(t, x),+}^{-k}(x)\right)\left[f_{+}^{-k}(x)-f_{t,+}^{-k}(x)\right] d x,
\end{aligned}
$$

for $u=u(t, x) \in[0, t]$. To finish, we shall next prove that the sum over $1 \leq k \leq M$ of (149) divided by $t$ converges as $t \rightarrow 0$ and $(M, t)$ is an admissible pair.

Recalling Definition (17) of $Y_{k, s}$, the proof of Lemma 6.1 (in particular (162)) implies that there is $s=s(t, x) \in[0, t]$ so that

$$
f_{t,+}^{-k}(x)-f_{+}^{-k}(x)=-t \frac{Y_{k, s}\left(f_{s,+}^{-k}(x)\right)}{\left(f_{s}^{k}\right)^{\prime}\left(f_{s,+}^{-k}(x)\right)}=-t \sum_{j=1}^{k} \frac{X_{s}\left(f_{s}^{j}\left(f_{s,+}^{-k}(x)\right)\right)}{\left(f_{s}^{j}\right)^{\prime}\left(f_{s,+}^{-k}(x)\right)} .
$$

Since $X$ is $C^{1}$, and since bounded distortion holds for points which climb (Lemma 3.3), we find (recall (46) in the proof of Proposition 3.9)

$$
\begin{array}{r}
\left|\sum_{j=1}^{k} \frac{X_{s}\left(f_{s,+}^{j-k}(x)\right)}{\left(f_{s}^{j}\right)^{\prime}\left(f_{s,+}^{-k}(x)\right)}-\frac{1}{f_{s}^{\prime}\left(f_{s,+}^{-k}(x)\right)} \sum_{j=1}^{k} \frac{X_{s}\left(f_{s,+}^{j-1}\left(c_{1, s}\right)\right)}{\left(f_{s}^{j-1}\right)^{\prime}\left(c_{1, s}\right)}\right| \\
\leq C e^{2 \gamma k}\left|\left(f_{s}^{k-1}\right)^{\prime}\left(c_{1, s}\right)\right|^{-1 / 2} .
\end{array}
$$

Then, using horizontality, we find

$$
\sum_{j=1}^{k} \frac{X_{s}\left(f_{s,+}^{j-1}\left(c_{1, s}\right)\right)}{\left(f_{s}^{j-1}\right)^{\prime}\left(c_{1, s}\right)}=-\frac{1}{\left(f_{s}^{k}\right)^{\prime}\left(c_{1, s}\right)} \sum_{\ell=k}^{\infty} \frac{X_{s}\left(f_{s}^{\ell}\left(c_{1, s}\right)\right)}{\left(f_{s}^{\ell-k}\right)^{\prime}\left(c_{k+1, s}\right)} .
$$

The proof of Proposition 3.9 implies that the above expression is bounded, uniformly in $k \leq M$ and admissible pairs $(M, t)$. (Here we use the uniform bounds from Lemma 5.8.) Finally, recalling the properties of the support of $\phi_{M, k}$

$$
\begin{aligned}
\left|\int_{c_{k}-e^{-\beta_{1} k}}^{c_{k, t}} \frac{1}{\left|\left(f_{s}^{\prime}\right)\left(f_{s,+}^{-k}(x)\right)\right|} d x\right| & \leq C \int_{c_{k}-C e^{-\beta_{1} k}}^{c_{k, t}} \frac{1}{\sqrt{\left|x-c_{k, s}\right|}} d x \\
& =\left.C \sqrt{\left|x-c_{k, s}\right|}\right|_{c_{k}-C e^{-\beta_{1} k}} ^{c_{k, t}}
\end{aligned}
$$

Summarizing, we have proved that (149) divided by $t$ satisfies (recall also Lemma 2.10 and (108))

$$
\begin{gathered}
\left|\frac{1}{t} \int_{-1}^{c_{k, t}} \lambda^{k} A^{\prime}(x) \phi_{M, k}\left(f_{u(t, x),+}^{-k}(x)\right)\left[f_{+}^{-k}(x)-f_{t,+}^{-k}(x)\right] d x\right| \\
=\left|\int_{-1}^{c_{k, t}} \lambda^{k} A^{\prime}(x) \phi_{M, k}\left(f_{u(t, x),+}^{-k}(x)\right) \frac{Y_{k, s}\left(f_{s,+}^{-k}(x)\right)}{\left(f_{s}^{k}\right)^{\prime}\left(f_{s,+}^{-k}(x)\right)} d x\right| \\
\leq C \kappa_{M}^{-k} \sup \left|A^{\prime}\right| \sup \left|\phi_{M, 0}\right| e^{-\beta_{1} k / 2} .
\end{gathered}
$$

The bound in the third line above is summable over $k \geq 1$, uniformly in $M$. 
Since it is easy to check for each fixed $k$ that

$$
\begin{aligned}
\lim _{t \rightarrow 0} \int_{-1}^{c_{k, t}} \lambda^{k} A^{\prime}(x) & \phi_{M, k}\left(f_{u(t, x),+}^{-k}(x)\right) \frac{Y_{k, s}\left(f_{s,+}^{-k}(x)\right)}{\left(f_{s}^{k}\right)^{\prime}\left(f_{s,+}^{-k}(x)\right)} d x \\
= & \int_{-1}^{c_{k}} \lambda^{k} A^{\prime}(x) \phi_{k}\left(f_{+}^{-k}(x)\right) \frac{Y_{k}\left(f_{+}^{-k}(x)\right)}{\left(f^{k}\right)^{\prime}\left(f_{+}^{-k}(x)\right.} d x,
\end{aligned}
$$

we have proved that

$$
\begin{aligned}
\lim _{t \rightarrow 0} & \frac{1}{t} \int_{I} A(x)\left(\Pi_{t}\left(\phi_{M}\right)-\Pi\left(\phi_{M}\right)\right)(x) d x \\
& =-\sum_{k=1}^{\infty} \sum_{\varsigma \in\{+,-\}} \pm \int_{-1}^{c_{k}} \lambda^{k} A^{\prime}(x) \phi_{k}\left(f_{+}^{-k}(x)\right) \frac{Y_{k}\left(f_{\varsigma}^{-k}(x)\right)}{\left(f^{k}\right)^{\prime}\left(f_{\varsigma}^{-k}(x)\right.} d x \\
& =-\sum_{k=1}^{\infty} \sum_{\varsigma \in\{+,-\}} \int_{-1}^{c_{k-1}} A^{\prime}(f(y)) \frac{\lambda^{k-1}}{\mid\left(f^{k-1}\right)^{\prime}\left(f_{\varsigma}^{-(k-1)}(y) \mid\right.} \lambda\left(\phi_{k} \cdot Y_{k}\right)\left(f_{\varsigma}^{-(k-1)}(y)\right) d y .
\end{aligned}
$$

(The sign in the second line above comes from in (145), that is, it is the sign of $\left(f^{k-1}\right)^{\prime}\left(f_{\varsigma}^{-(k-1)}(x)\right.$.) The fixed point property of $\hat{\phi}$ implies $\lambda \phi_{k+1}=\phi_{k} \xi_{k}$. Therefore, setting $\hat{\xi}=\left(\xi_{k}\right)$, and recalling the shift in indices in the Definition (18) of $\hat{Y}$, we have

$$
\begin{aligned}
\lim _{t \rightarrow 0} \frac{1}{t} \int_{I} A(x)\left(\Pi_{t}\left(\hat{\phi}_{M}\right)-\Pi\left(\hat{\phi}_{M}\right)\right)(x) d x & =-\int_{I}\left(A^{\prime} \circ f\right) \cdot \Pi(\hat{\xi} \hat{Y} \hat{\phi}) d y \\
& =-\lambda \int_{I} A^{\prime} \cdot\left(\Pi \circ\left(\mathrm{id}-\mathcal{J}_{0}\right) \circ \hat{\mathscr{L}}\right)(\hat{Y} \hat{\phi}) d y,
\end{aligned}
$$

where we used (78). This ends Step 3 and the proof of Theorem 2.13.

\section{Appendix A}

\section{Relating the conjugacies $h_{t}$ with the infinitesimal conjugacy $\alpha$}

We show here that $\alpha$ deserves to be called an infinitesimal conjugacy.

Proof of Proposition 2.15. - Let $\alpha_{t}:[-1,1] \rightarrow \mathbb{R}$ be the unique continuous solution for the TCE

$$
v_{t}=\alpha_{t} \circ f_{t}-f_{t}^{\prime} \cdot \alpha_{t}
$$

Since the family $\left\{\alpha_{t}\right\}_{|t|<\epsilon}$ is equicontinuous and the solutions are unique, it is easy to see that $(t, x) \rightarrow \alpha_{t}(x)$ is a continuous and bounded function in $(-\epsilon, \epsilon) \times[-1,1]$. Note that $\alpha_{t}(-1)=\alpha_{t}(1)=0$ for every $t$ since $f_{t}( \pm 1)=-1$ for all $t$. For each $x_{0} \in[-1,1]$ and $t_{0} \in(-\epsilon, \epsilon)$, the Peano theorem ensures that the ODE

$$
\left.\partial u_{s}\left(t_{0}, x_{0}\right)\right|_{t=s}=\alpha_{t}\left(u_{t}\left(t_{0}, x_{0}\right)\right), \quad u_{t_{0}}\left(t_{0}, x_{0}\right)=x_{0}
$$

admits a $C^{1}$ solution $u_{t}\left(t_{0}, x_{0}\right)$. It is not difficult to see that this solution is defined for every $t \in(-\epsilon, \epsilon)$. Since $f_{t}$ is a deformation, there exists a unique conjugacy $h_{t}$ such that

$$
f_{t} \circ h_{t}=h_{t} \circ f_{0} .
$$

If $x_{t_{0}}$ is an eventually periodic point for $f_{t_{0}}$, since all periodic points are hyperbolic there exists an analytic continuation $x_{t}$ for $x_{0}$. Then $x_{t}=h_{t}\left(x_{0}\right)$. An easy calculation shows that $u_{t}\left(t_{0}, x_{0}\right)=h_{t}\left(x_{0}\right)$ is a solution of the above ODE. 
Claim: If $w_{t}$ is a solution of the ODE $\partial_{t} w_{t}=\alpha_{t}\left(w_{t}\right)$ and $w_{t_{0}}=h_{t_{0}}\left(x_{0}\right)$ for some $t_{0}$ and eventually periodic point $x_{0}$ then $w_{t}=h_{t}\left(x_{0}\right)$ for every $t$. Indeed, denote $w_{t}^{n}=f_{t}^{n}\left(w_{t}\right)$. Note that the TCE implies that $w_{t}^{n}$ and $f_{t}^{n}\left(h_{t}\left(x_{0}\right)\right)$ are also solutions of the ODE above. Since $\alpha_{t}(x)$ is a bounded function

$$
\left|w_{t}^{n}-f_{t}^{n}\left(h_{t}\left(x_{0}\right)\right)\right| \leq\left|w_{t}^{n}-w_{t_{0}}^{n}\right|+\left|f_{t_{0}}^{n}\left(h_{t_{0}}\left(x_{0}\right)\right)-f_{t}^{n}\left(h_{t}\left(x_{0}\right)\right)\right| \leq 2 \sup _{(t, x)}\left|\alpha_{t}(x)\right|\left|t-t_{0}\right| .
$$

So if $t$ is sufficiently close to $t_{0}$ then

$$
\left|f_{t}^{n}\left(w_{t}\right)-f_{t}^{n}\left(h_{t}\left(x_{0}\right)\right)\right|<\delta
$$

for every $n$. If $\delta$ is small enough, since the orbit of $h_{t}\left(x_{0}\right)$ by $f_{t}$ eventually lands on a repelling periodic point, it follows that $w_{t}=h_{t}\left(x_{0}\right)$ for $t$ close enough to $t_{0}$. This argument implies that

$$
\left\{t: w_{t}=h_{t}\left(x_{0}\right)\right\}
$$

is an open set in $(-\epsilon, \epsilon)$. Since it is obviously a closed set, it follows that $w_{t}=h_{t}\left(x_{0}\right)$ for every $t$. This finishes the proof of the claim.

In particular this claim implies the uniqueness of the solution of the ODE when $x_{0}$ is an eventually periodic point.

Now let $x$ be a point that is not eventually periodic for $f_{0}$. We can find sequences $p_{n}, q_{n}$ of eventually periodic points for $f_{0}$ such that

$$
p_{n}<x<q_{n}
$$

and $\lim _{n} p_{n}=\lim _{n} q_{n}=x$. Let $w_{t}$ be a solution for $\partial_{t} w_{t}=\alpha_{t}\left(w_{t}\right)$ such that $w_{0}=x$. The claim above implies that

$$
h_{t}\left(p_{n}\right)<w_{t}<h_{t}\left(q_{n}\right)
$$

for every $n$. Since $h_{t}$ is continuous we get $\lim _{n} h_{t}\left(p_{n}\right)=\lim _{n} h_{t}\left(q_{n}\right)=h_{t}(x)$, so $h_{t}(x)=w_{t}$, for every $t$.

\section{Appendix B}

\section{The Lasota-Yorke bound [15, Sublemma] for Sobolev norms and probabilistic operators}

We adapt the argument of [15, Sublemma] (see also [59, Lemma 5.5]) to complete the proof of Proposition 4.10, by showing that there exists $C$, and for all $n$ there is $C(n)$, so that

$$
\left\|\left(\widehat{\mathscr{L}}^{n}(\hat{\psi})\right)_{0}^{\prime}\right\|_{L^{1}(I)} \leq C \Theta_{0}^{-n}\|\hat{\psi}\|_{\mathscr{B}}+C(n)\|\hat{\psi}\|_{\mathscr{B}^{L^{1}}} .
$$

Keeping in mind (83) and (84), as well as the properties of the supports of the $\xi_{j}$ and $\psi_{j},(67)$, (66), (80), (81), and the conditions (70) on $\lambda$, one first obtains the following analogue of [15, (4.14)-(4.15)] or [59, (5.25)-(5.26)]: There is a constant $C$ and for each $n$ a constant $C(n)$ so that for each interval $A \subset E_{0}$

$$
\begin{aligned}
\int_{A}\left|\left(\hat{\mathcal{L}}^{n}(\hat{\psi})\right)_{0}^{\prime}(x)\right| d x & \leq C \frac{\Theta^{-n}}{c(\delta)}\left(\sum_{\omega \in \Omega_{0}} \int_{\omega}\left|\hat{\psi}^{\prime}(x)\right| d x+\|\hat{\psi}\|_{\mathcal{B}^{W_{1}^{1}}}\right) \\
& +\frac{C(n)}{c(\delta)}\|\hat{\psi}\|_{\mathcal{B}^{L^{1}}}+C \sum_{\ell=H(\delta)}^{n-1} \frac{\Theta^{-\ell}}{c(\delta)} \sum_{\omega^{\prime} \in \Omega_{\ell}} \int_{\omega^{\prime}}\left|\left(\hat{\mathscr{L}}^{n-\ell}(\hat{\psi})\right)^{\prime}(x)\right| d x,
\end{aligned}
$$


while if the interval $A \subset(-\delta, \delta)$, we get a stronger estimate (by Lemma 3.5) where all factors $c(\delta)$ in the right-hand side above may be replaced by 1 .

In (152), the intervals $\omega$ in $\Omega_{0}$ are either inverse images of $A$ in $E_{0}$ through branches of our probabilistic version of $\hat{f}^{n}$ which always stay in $E_{0}$, or inverse image of $A$ in $E_{k}, k(\omega) \geq 0$, through branches of the probabilistic $\hat{f}^{n}$ which start from $E_{k}$, climb to $E_{k+n-j}$, for $n-j-1$ iterations with $0 \leq j \leq n-1$, then drop to level $E_{0}$ and stay there for the last $j$ iterations. (The detailed analysis is slightly different depending on whether $k(\omega)>N$ or $\leq N$, for some $N$ to be chosen much larger than $n$, in order to avoid dividing by small lengths $|U|$ in (80), see [15] or [59] for details.) The intervals $\omega \in \Omega_{0}$ in $E_{k}$ for $k \geq 0$ which will drop from $E_{k+n-j}$ for $k+n-j \geq H(\delta)$ have intersection multiplicity at most $k+n-j$ by Remark 4.9. Since the corresponding $k+n-j$ factor is killed by an exponential $\rho^{-(k+n-j)}$ factor implicit in (152) (see [15, Sublemma]), we may in fact glue overlapping intervals together up to taking a slightly smaller $\Theta>1$. The intervals $\omega \in \Omega_{0}$ whose orbits never leave $E_{0}$ are disjoint by construction. Each of them meets at most one of the (just grouped) intervals which go through $E_{k+n-j}$, and we can glue them together at the cost of replacing $C$ by $C+C=2 C$.

The intervals $\omega^{\prime} \in \Omega_{\ell}$ in (152) are inverse images of $A$ in $E_{0}$ via the branches of our probabilistic version of $\hat{f}^{\ell}$ which climb the tower up to some level $k=k\left(\omega^{\prime}\right)$ with $H(\delta) \leq k<\ell<n<N$, fall to level $E_{0}$, and then stay in $E_{0}$ for the remaining $\ell-k-1$ iterations. Remark 4.9 about the maximum overlap of fuzzy monotonicity intervals $\tilde{I}_{k}$ ensures that the intersection multiplicity of the intervals in $\Omega_{\ell}$ dropping from level $k \geq H(\delta)$ is at most $k \leq \ell$. Since $\ell \Theta^{-\ell}$ may be replaced by $\Theta^{-\ell}$ for $\ell \geq H(\delta)$, up to taking a slightly smaller $\Theta$, we may regroup overlapping intervals in $\Omega_{\ell}$.

If $\Omega_{\ell}$ is empty for $\ell \geq 1$, we are done. Otherwise, to perform the inductive step, let us rename $\Omega_{\ell}=\Omega_{\ell}^{1}$ for $\ell \geq 0$. Exploiting Lemma 3.5 to see that we get at most one factor $c(\delta)^{-1}$, we can then inductively conclude the argument, just like in [15, Sublemma] (see also [59, Lemma 5.5]). The only difference with respect to the analysis in [15] is that the intervals of $\Omega_{0}^{2}$ (after regrouping, which may be done as above) may overlap with those of $\Omega_{0}^{1}$. More generally, the intervals of $\Omega_{0}^{m+1}$ may intersect those of $\cup_{i=1}^{m} \Omega_{0}^{i}$. Since there are at most $n / H(\delta)$ inductive steps, the overlap factor $n / H(\delta)$ is negligible in front of $C \Theta^{-n}$.

\section{Appendix C}

\section{Proof of Lemma 6.1 on Taylor expansions}

As usual, we consider $f_{+}^{-k}$, the other branch is similar. The assumptions imply that $\left.\partial_{t} f_{t}\right|_{t=s}=X_{s} \circ f_{s}$, where $X_{s} \circ f_{s}$ is $C^{2}$ and horizontal for $f_{s}$.

We prove (134) and (135)-(136) for $s=0$, the general case then follows from Lemma 5.8, using that for all $H_{0} \leq k \leq M$ so that (108) holds, we may take $\xi_{k, t}=\xi_{k}$ by Proposition 5.9.

By horizontality, the estimate (46) in the proof of Proposition 3.9 (using the notation $w_{k}(y)$ introduced there) gives $C>0$ so that for any $k \geq H_{0}$

$$
\sup _{y \in I_{k}} \frac{\left|Y_{k}(y)\right|}{\left|\left(f^{k}\right)^{\prime}(y)\right|}=\left|\sup _{y \in I_{k}} \sum_{j=1}^{k} \frac{X\left(f^{j}(y)\right)}{\left(f^{j}\right)^{\prime}(y)}\right|=\sup _{y \in I_{k}}\left|w_{k}(y)\right| \leq C \frac{e^{2 \gamma k}}{\left|\left(f^{k-1}\right)^{\prime}\left(c_{1}\right)\right|^{1 / 2}},
$$

proving (134). 
For the claim (135) on the derivative, note that

$$
\partial_{x} \frac{Y_{k}\left(f_{+}^{-k}(x)\right)}{\left(f^{k}\right)^{\prime}\left(f_{+}^{-k}(x)\right)}=\frac{1}{\left(f^{k}\right)^{\prime}\left(f_{+}^{-k}(x)\right)} \partial_{y} \frac{Y_{k}(y)}{\left(f^{k}\right)^{\prime}(y)},
$$

with

$$
\begin{aligned}
\partial_{y} \frac{Y_{k}(y)}{\left(f^{k}\right)^{\prime}(y)} & =\sum_{j=1}^{k} \partial_{y} \frac{X\left(f^{j}(y)\right)}{\left(f^{j}\right)^{\prime}(y)} \\
& =\sum_{j=1}^{k} X^{\prime}\left(f^{j}(y)\right)-\sum_{j=1}^{k} X\left(f^{j}(y)\right) \sum_{\ell=0}^{j-1} \frac{f^{\prime \prime}\left(f^{\ell}(y)\right)}{\left(f^{j-\ell}\right)^{\prime}\left(f^{\ell}(y)\right) f^{\prime}\left(f^{\ell}(y)\right)}
\end{aligned}
$$

We shall use the estimates in the proof of Lemma 4.1: For $y \in I_{k}$, the bound (64) says that $\left|\left(f^{k-m}\right)^{\prime}\left(f^{m}(y)\right)\right| \geq C e^{-\gamma m}$, for $1 \leq m \leq k-1$, the bound (65) says that $\left|\left(f^{m}\right)^{\prime}(y)\right| \geq$ $C_{2} e^{-\gamma k}\left|\left(f^{m-1}\right)^{\prime}\left(c_{1}\right)\right|\left|\left(f^{k-1}\right)^{\prime}\left(c_{1}\right)\right|^{-1 / 2}$ for $1 \leq m \leq k$, while (63) gives $\left|f^{\prime}\left(f^{\ell}(y)\right)\right| \geq C e^{-\gamma \ell}$ for $1 \leq \ell \leq k$. (These bounds do not use horizontality.)

The second term in the right-hand side of (155) can be decomposed as

$$
\begin{aligned}
& -\sum_{j=1}^{k} X\left(f^{j}(y)\right) \sum_{\ell=0}^{j-1} \frac{f^{\prime \prime}\left(f^{\ell}(y)\right)}{\left(f^{j-\ell}\right)^{\prime}\left(f^{\ell}(y)\right) f^{\prime}\left(f^{\ell}(y)\right)} \\
& \quad=-\frac{f^{\prime \prime}(y)}{f^{\prime}(y)} \sum_{j=1}^{k} \frac{X\left(f^{j}(y)\right)}{\left(f^{j}\right)^{\prime}(y)}-\sum_{\ell=1}^{k-1} \frac{f^{\prime \prime}\left(f^{\ell}(y)\right)}{f^{\prime}\left(f^{\ell}(y)\right)} \sum_{j=\ell+1}^{k} \frac{X\left(f^{j}(y)\right)}{\left(f^{j-\ell}\right)^{\prime}\left(f^{\ell}(y)\right)} .
\end{aligned}
$$

By (65) for $m=1$, combined with (134) (which holds by horizontality), we find

$$
\left|\frac{f^{\prime \prime}(y)}{f^{\prime}(y)} \sum_{j=1}^{k} \frac{X\left(f^{j}(y)\right)}{\left(f^{j}\right)^{\prime}(y)}\right| \leq C e^{3 \gamma k}
$$

The second term in the right-hand side of (156) does not require horizontality, only (64) and (63), which give

$$
\sum_{\ell=1}^{k-1} \frac{\left|f^{\prime \prime}\left(f^{\ell}(y)\right)\right|}{\left|f^{\prime}\left(f^{\ell}(y)\right)\right|} \sum_{j=\ell+1}^{k} \frac{\left|X\left(f^{j}(y)\right)\right|}{\left|\left(f^{j-\ell}\right)^{\prime}\left(f^{\ell}(y)\right)\right|} \leq C e^{2 \gamma k}
$$

Remembering (154), and using again (65) (for $m=k$ ), we have proved (135).

The proof of (136) is similar. We start by noting that $\partial_{x}^{2} \frac{Y_{k}\left(f_{+}^{-k}(x)\right)}{\left(f^{k}\right)^{\prime}\left(f_{+}^{-k}(x)\right)}=$

$$
\partial_{x} \frac{1}{\left(f^{k}\right)^{\prime}\left(f_{+}^{-k}(x)\right)} \partial_{y} \frac{Y_{k}(y)}{\left(f^{k}\right)^{\prime}(y)}+\frac{1}{\left(\left(f^{k}\right)^{\prime}\left(f_{+}^{-k}(x)\right)^{2}\right.} \partial_{y}^{2} \frac{Y_{k}(y)}{\left(f^{k}\right)^{\prime}(y)}
$$


where $\partial_{y}^{2} \frac{Y_{k}(y)}{\left(f^{k}\right)^{\prime}(y)}=$

$$
\begin{aligned}
\sum_{j=1}^{k} & X^{\prime \prime}\left(f^{j}(y)\right)\left(f^{j}\right)^{\prime}(y)-\sum_{j=1}^{k} X^{\prime}\left(f^{j}(y)\right) \sum_{\ell=0}^{j-1} \frac{f^{\prime \prime}\left(f^{\ell}(y)\right)\left(f^{\ell}\right)^{\prime}(y)}{f^{\prime}\left(f^{\ell}(y)\right)} \\
& -\sum_{j=1}^{k} X\left(f^{j}(y)\right)\left[\sum_{\ell=0}^{j-1} \frac{f^{\prime \prime \prime}\left(f^{\ell}(y)\right)\left(f^{\ell}\right)^{\prime}(y)}{\left(f^{j-\ell}\right)^{\prime}\left(f^{\ell}(y)\right) f^{\prime}\left(f^{\ell}(y)\right)}-\frac{f^{\prime \prime}\left(f^{\ell}(y)\right)^{2}\left(f^{\ell}\right)^{\prime}(y)}{\left(f^{j-\ell}\right)^{\prime}\left(f^{\ell}(y)\right)\left(f^{\prime}\left(f^{\ell}(y)\right)\right)^{2}}\right. \\
& \left.-\frac{f^{\prime \prime}\left(f^{\ell}(y)\right)}{f^{\prime}\left(f^{\ell}(y)\right)} \sum_{i=0}^{j-\ell-1} \frac{f^{\prime \prime}\left(f^{i}(y)\right)}{\left(f^{j-\ell-i}\right)^{\prime}\left(f^{\ell+i}(y)\right) f^{\prime}\left(f^{\ell+i}(y)\right)}\right]
\end{aligned}
$$

The first term in (158) is bounded by $e^{5 \gamma k}\left|\left(f^{k-1}\right)^{\prime}\left(c_{1}\right)\right|^{-1 / 2}$ by the proof of (135) and (59) from Lemma 4.1. The first term in (159) is bounded by $C\left|\left(f^{k-1}\right)^{\prime}\left(c_{1}\right)\right|^{1 / 2}$, in view of Lemma 3.3 and Lemma 3.4. Hence, dividing by $\left(\left(f^{k}\right)^{\prime}\left(f_{+}^{-k}(x)\right)^{2}\right.$, and using Lemma 3.4, the contribution of this term is bounded by $C e^{2 \gamma k}\left|\left(f^{k-1}\right)^{\prime}\left(c_{1}\right)\right|^{-1 / 2}$.

Using again the same observations, we find that the second term in (159) is bounded by $C e^{3 \gamma k}$. Dividing by $\left(\left(f^{k}\right)^{\prime}\left(f_{+}^{-k}(x)\right)^{2}\right.$, we get a contribution bounded by $C e^{5 \gamma k}\left|\left(f^{k-1}\right)^{\prime}\left(c_{1}\right)\right|^{-1}$.

For (160), one must distinguish between the terms for $\ell=0$, for which horizontality gives a bound $C e^{4 \gamma k}\left|\left(f^{k-1}\right)^{\prime}\left(c_{1}\right)\right|^{1 / 2}$ (note the factor $\left(f^{\prime}(y)\right)^{2}$ in the denominator), and the terms where $\ell \geq 1$, for which a straightforward estimate, using the remarks above (and in particular Lemma 3.3), gives an upper bound of the form $C e^{3 \gamma k}$. Dividing by $\left(\left(f^{k}\right)^{\prime}\left(f_{+}^{-k}(x)\right)^{2}\right.$, we get a contribution bounded by $C e^{5 \gamma k}\left|\left(f^{k-1}\right)^{\prime}\left(c_{1}\right)\right|^{-1 / 2}$.

For (161), if $\ell=0$ and $i=0$, horizontality gives a bound $C e^{4 \gamma k}\left|\left(f^{k-1}\right)^{\prime}\left(c_{1}\right)\right|^{1 / 2}$, while if $i+\ell \geq 1$, we get a bound $C e^{4 \gamma k}$. Dividing by $\left(\left(f^{k}\right)^{\prime}\left(f_{+}^{-k}(x)\right)^{2}\right.$, we get a contribution $\leq C e^{6 \gamma k}\left|\left(f^{k-1}\right)^{\prime}\left(c_{1}\right)\right|^{-1 / 2}$. This ends the proof of (136).

In view of the more complicated estimates to follow, we notice the following pattern: The dangerous factors in the above estimates are powers of $f^{\prime}(y)$ in the denominator and factors $\left(f^{\ell}\right)^{\prime}(y)$ for large $\ell$ in the numerator. The "white knight" available to fight them is a power of $\left(f^{k}\right)^{\prime}(y)$ in the denominator. An additional such power appears each time we differentiate with respect to $x$. The terms for which the power of $f^{\prime}(y)$ in the denominator exceeds that of $\left(f^{k}\right)^{\prime}(y)$ in the numerator can be handled by horizontality. The price to be paid for the control is a power of $e^{\gamma k}$.

We turn to (137) and (138). If $(x, t) \mapsto \Phi_{t}(x) \in I$ is a $C^{1}$ map on $I \times[-\epsilon, \epsilon]$ so that $x \mapsto \Phi_{t}(x)$ is invertible, then we have

$$
\left.\partial_{t} \Phi_{t}^{-1}(x)\right|_{t=s}=-\frac{\left(\left.\partial_{t} \Phi_{t}\right|_{t=s}\right) \circ \Phi_{s}^{-1}(x)}{\left(\partial_{x} \Phi_{s}\right) \circ \Phi_{s}^{-1}(x)}
$$


and

(163)

$$
\begin{aligned}
\left.\partial_{t t}^{2} \Phi_{t}^{-1}(x)\right|_{t=s}=\left.\partial_{t} \frac{\left(\partial_{t} \Phi_{t}\right) \circ \Phi_{t}^{-1}(x)}{\left(\partial_{x} \Phi_{t}\right) \circ \Phi_{t}^{-1}(x)}\right|_{t=s} \\
=\frac{1}{\left(\partial_{x} \Phi_{s}\right) \circ \Phi_{s}^{-1}(x)} \cdot\left(\left.\partial_{t t}^{2} \Phi_{t}\right|_{t=s} \circ \Phi_{s}^{-1}(x)+\left.\left.\partial_{x t}^{2} \Phi_{t}\right|_{t=s} \circ \Phi_{s}^{-1}(x) \cdot \partial_{t} \Phi_{t}^{-1}(x)\right|_{t=s}\right) \\
\quad-\frac{\left(\left.\partial_{t} \Phi_{t}\right|_{t=s}\right) \circ \Phi_{s}^{-1}(x)}{\left(\partial_{x} \Phi_{s}\right) \circ \Phi_{s}^{-1}(x)} \frac{\left(\left.\partial_{t x}^{2} \Phi_{s}\right|_{t=s}\right) \circ \Phi_{s}^{-1}(x)+\left.\left(\partial_{x x}^{2} \Phi_{s}\right) \circ \Phi_{s}^{-1}(x) \cdot \partial_{t} \Phi_{t}^{-1}(x)\right|_{t=s}}{\left(\partial_{x} \Phi_{s}\right) \circ \Phi_{s}^{-1}(x)} .
\end{aligned}
$$

Since $t \mapsto f_{t} \in C^{2}(I)$ is $C^{2}$, we may apply the above to $\Phi_{t}(x)=f_{t}^{k}(x)$ restricted to a suitable domain. The right-hand side of (162) is just $Y_{k, s}\left(f_{s,+}^{-k}(x)\right) /\left(f_{s}^{k}\right)^{\prime}\left(f_{s,+}^{-k}(x)\right)$. Then, a Taylor series of order 2 gives

$$
f_{+}^{-k}(x)-f_{t,+}^{-k}(x)=t \frac{Y_{k}\left(f_{+}^{-k}(x)\right)}{\left(f^{k}\right)^{\prime}\left(f_{+}^{-k}(x)\right)}+t^{2} F_{k}(x, s),
$$

where $x$ is as in (137) and $s \in[0, t]$. In order to estimate $F_{k}(x, s)$, we look at the various terms in (163). The (identical) factors $\partial_{t} \Phi_{t}^{-1}$ and $\left(\partial_{t} \Phi_{t} / \partial_{x} \Phi_{t}\right) \circ \Phi_{t}^{-1}$ can be bounded by (134). Since $\left.\partial_{x t}^{2} \Phi_{t}\right|_{t=0}=\partial_{y} Y_{k}$, the two terms containing this expression can be controlled, when divided by $\left(\partial_{x} \Phi_{t}\right) \circ \Phi_{t}^{-1}$, respectively by $e^{-2 \gamma k}\left|\left(f^{k}\right)^{\prime}\left(c_{1}\right)\right|^{1 / 2}$, by using the ideas to bound (155). Analyzing the term containing $\partial_{x x}^{2} \Phi_{t}$ is of the same type as (but simpler than) what we did for $\partial_{y y}^{2} Y_{k}$, and the available factor $\left|\left(f^{k}\right)^{\prime}\left(c_{1}\right)\right|^{-1}$ gives the right control. The only new expression is

$$
\left.\partial_{t t}^{2} \Phi_{t}\right|_{t=s}(x)=Z_{k, s}(x):=\left.\partial_{t} Y_{k, t}(x)\right|_{t=s}=\lim _{t \rightarrow s} \frac{Y_{k, t}(x)-Y_{k, s}(x)}{t-s} .
$$

This involves functions such as $f^{\prime}, f^{\prime \prime}, f^{\prime \prime}, X_{s}$, and $X_{s}^{\prime}$, but also $\partial_{t} X_{t}$. The dominant term contains a factor $\mid\left(f^{k}\right)^{\prime}(y)$, which can be controlled by $\left(\partial_{x} \Phi_{s}\right) \circ \Phi_{s}^{-1}$ in the denominator.

Finally, using

$$
\frac{1}{\left(f_{t}^{k}\right)^{\prime}\left(f_{+}^{-k}(x)\right)}-\frac{1}{\left(f^{k}\right)^{\prime}\left(f_{t,+}^{-k}(x)\right)}=\left(f_{t,+}^{-k}(x)-f_{+}^{-k}(x)\right)^{\prime},
$$

we find

$$
\frac{1}{\left(f_{t}^{k}\right)^{\prime}\left(f_{+}^{-k}(x)\right)}-\frac{1}{\left(f^{k}\right)^{\prime}\left(f_{t,+}^{-k}(x)\right)}=t\left(\frac{Y_{k}\left(f_{+}^{-k}(x)\right)}{\left(f^{k}\right)^{\prime}\left(f_{+}^{-k}(x)\right)}\right)^{\prime}+t^{2} G_{k}(x, s),
$$

for $x$ as in (138) and $s \in[0, t]$. The new derivatives appearing in $G_{k}$ are $\partial_{x x x}^{3} \Phi, \partial_{t x x}^{3} \Phi$ and $\partial_{t t x}^{3} \Phi$ (but not $\partial_{t t t}^{3} \Phi$, which is a priori undefined). The claimed estimates on $\sup \left|G_{k}\right|$ can be obtained by horizontality, similarly to those for $F_{k}$, using now the $x$-derivative of (163) and exploiting in addition to the previous remarks the bound (136). The cancellation pattern described above emerges again. The computations are straightforward and left to the reader.

\section{REFERENCES}

[1] J. F. Alves, M. Carvalho, J. M. Freitas, Statistical stability and continuity of SRB entropy for systems with Gibbs-Markov structures, Comm. Math. Phys. 296 (2010), 739-767. 
[2] J. F. Alves, M. Carvalho, J. M. Freitas, Statistical stability for Hénon maps of the Benedicks-Carleson type, Ann. Inst. H. Poincaré Anal. Non Linéaire 27 (2010), 595637.

[3] A. Avila, Infinitesimal perturbations of rational maps, Nonlinearity 15 (2002), 695704.

[4] A. Avila, M. Lyubich, W. De Melo, Regular or stochastic dynamics in real analytic families of unimodal maps, Invent. Math 154 (2003), 451-550.

[5] A. Avila, C. G. Moreira, Bifurcations of unimodal maps, in Dynamical systems. Part II, Pubbl. Cent. Ric. Mat. Ennio Giorgi, Scuola Norm. Sup., 2003, 1-22.

[6] A. Avila, C. G. Moreira, Phase-parameter relation and sharp statistical properties for general families of unimodal maps, in Geometry and dynamics, Contemp. Math. 389, Amer. Math. Soc., 2005, 1-42.

[7] V. BALAdi, On the susceptibility function of piecewise expanding interval maps, Comm. Math. Phys. 275 (2007), 839-859.

[8] V. BALADI, Linear response despite critical points, Nonlinearity 21 (2008), T81-T90.

[9] V. Baladi, Y. Jiang, H. H. Rugh, Dynamical determinants via dynamical conjugacies for postcritically finite polynomials. Dedicated to David Ruelle and Yasha Sinai on the occasion of their 65th birthdays, J. Statist. Phys. 108 (2002), 973-993.

[10] V. Baladi, D. Smania, Linear response formula for piecewise expanding unimodal maps, Nonlinearity 21 (2008), 677-711.

[11] V. Baladi, D. Smania, Analyticity of the SRB measure for holomorphic families of quadratic-like Collet-Eckmann maps, Proc. Amer. Math. Soc. 137 (2009), 14311437.

[12] V. Baladi, D. Smania, Smooth deformations of piecewise expanding unimodal maps, Discrete Contin. Dyn. Syst. 23 (2009), 685-703.

[13] V. Baladi, D. Smania, Alternative proofs of linear response for piecewise expanding unimodal maps, Ergodic Theory and Dynam. Systems 30 (2010), 1-20.

[14] V. Baladi, D. Smania, Corrigendum to: Linear response formula for piecewise expanding unimodal maps, Nonlinearity 25 (2012), 2203-2205.

[15] V. Baladi, M. Viana, Strong stochastic stability and rate of mixing for unimodal maps, Ann. Sci. École Norm. Sup. 29 (1996), 483-517.

[16] M. Benedicks, L. Carleson, The dynamics of the Hénon map, Ann. of Math. 133 (1991), 73-169.

[17] H. Bruin, S. Luzzatto, S. Van Strien, Decay of correlations in one-dimensional dynamics, Ann. Sci. École Norm. Sup. 36 (2003), 621-646.

[18] H. Bruin, J. Rivera-Letelier, W. Shen, S. Van Strien, Large derivatives, backward contraction and invariant densities for interval maps, Invent. Math. 172 (2008), 509-533.

[19] O. Butterley, C. Liverani, Smooth Anosov flows: correlation spectra and stability, J. Mod. Dyn. 1 (2007), 301-322.

[20] B. CessaC, Does the complex susceptibility of the Hénon map have a pole in the upperhalf plane? A numerical investigation, Nonlinearity 20 (2007), 2883-2895. 
[21] D. Dolgopyat, On differentiability of SRB states for partially hyperbolic systems, Invent. Math. 155 (2004), 389-449.

[22] J. Graczyk, D. Sands, G. Świạtek, La dérivée schwarzienne en dynamique unimodale, C. R. Acad. Sci. Paris Sér. I Math. 332 (2001), 329-332.

[23] M. Hairer, A. J. Majda, A simple framework to justify linear response theory, Nonlinearity 23 (2010), 909-922.

[24] H. Hennion, Sur un théorème spectral et son application aux noyaux lipchitziens, Proc. Amer. Math. Soc. 118 (1993), 627-634.

[25] A. Katok, G. Knieper, M. Pollicott, H. Weiss, Differentiability and analyticity of topological entropy for Anosov and geodesic flows, Invent. Math. 98 (1989), 581597.

[26] G. Keller, Stochastic stability in some dynamical systems, Monatshefte Math. 94 (1982), 313-333.

[27] G. KeLLER, Exponents, attractors and Hopf decompositions for interval maps, Ergodic Theory Dynam. Systems 10 (1990), 717-744.

[28] G. Keller, P. J. Howard, R. Klages, Continuity properties of transport coefficients in simple maps, Nonlinearity 21 (2008), 1719-1743.

[29] G. Keller, C. Liverani, Stability of the spectrum for transfer operators, Ann. Scuola Norm. Sup. Pisa Cl. Sci. 28 (1999), 141-152.

[30] T. G. Keller, Nowicki, Spectral theory, zeta functions and the distribution of periodic points for Collet-Eckmann maps, Comm. Math. Phys. 149 (1992), 633-680.

[31] O. S. Kozlovski, Getting rid of the negative Schwarzian derivative condition, Ann. of Math. 152 (2000), 743-762.

[32] S. Luzzatto, L. WANG, Topological invariance of generic non-uniformly expanding multimodal maps, Math. Res. Lett. 13 (2006), 343-357.

[33] M. Martens, Distortion results and invariant Cantor sets of unimodal maps, Ergodic Theory Dynam. Systems 14 (1994), 331-349.

[34] M. Martens, W. De Melo, The multipliers of periodic points in one-dimensional dynamics, Nonlinearity 12 (1999), 217-227.

[35] M. Mazzolena, Dinamiche espansive unidimensionali: dipendenza della misura invariante da un parametro, Master's Thesis, Roma 2 (2007).

[36] W. De Melo, S. van Strien, One-dimensional dynamics, Ergebnisse Math. Grenzg., Springer, 1993.

[37] T. Nowicki, On some dynamical properties of $S$-unimodal maps on an interval, Fund. Math. 126 (1985), 27-43.

[38] T. Nowicki, Symmetric $S$-unimodal mappings and positive Liapunov exponents, Ergodic Theory Dynam. Systems 5 (1985), 611-616.

[39] T. Nowicki, Some dynamical properties of $S$-unimodal maps, Fund. Math. 142 (1993), 45-57.

[40] T. Nowicki, F. Przytycki, Topological invariance of the Collet-Eckmann property for S-unimodal maps, Fund. Math. 155 (1998), 33-43.

[41] T. Nowicki, D. SANDS, Non-uniform hyperbolicity and universal bounds for $S$-unimodal maps, Invent. Math. 132 (1998), 633-680.

$4^{\mathrm{e}}$ SÉRIE - TOME $45-2012-\mathrm{N}^{\circ} 6$ 
[42] T. Nowicki, S. van Strien, Hyperbolicity properties of $C^{2}$ multi-modal ColletEckmann maps without Schwarzian derivative assumptions, Trans. Amer. Math. Soc. 321 (1990), 793-810.

[43] T. Nowicki, S. van Strien, Invariant measures exist under a summability condition for unimodal maps, Invent. Math. 105 (1991), 123-136.

[44] F. Przytycki, J. Rivera-Letelier, Statistical properties of topological ColletEckmann maps, Ann. Sci. École Norm. Sup. 40 (2007), 135-178.

[45] D. Ruelle, Differentiation of SRB states, Comm. Math. Phys. 187 (1997), 227-241.

[46] D. Ruelle, General linear response formula in statistical mechanics, and the fluctuation-dissipation theorem far from equilibrium, Phys. Lett. A 245 (1998), 220-224.

[47] D. Ruelle, Differentiation of SRB states: Corrections and complements, Comm. Math. Phys. 234 (2003), 185-190.

[48] D. Ruelle, Application of hyperbolic dynamics to physics: some problems and conjectures, Bull. Amer. Math. Soc. 41 (2004), 275-278.

[49] D. Ruelle, Differentiating the absolutely continuous invariant measure of an interval map $f$ with respect to $f$, Comm. Math. Phys. 258 (2005), 445-453.

[50] D. RuElle, A review of linear response theory for general differentiable dynamical systems, Nonlinearity 22 (2009), 855-870.

[51] D. Ruelle, Structure and $f$-dependence of the A.C.I.M. for a unimodal map $f$ of Misiurewicz type, Comm. Math. Phys. 287 (2009), 1039-1070.

[52] D. Ruelle, Private communication by e-mail, 29 November 2009.

[53] M. Rychlik, E. Sorets, Regularity and other properties of absolutely continuous invariant measures for the quadratic family, Comm. Math. Phys. 150 (1992), 217236.

[54] D. SANDs, Topological conditions for positive Lyapunov exponent in unimodal maps, Ph.D. Thesis, St John's College, 1994.

[55] S. van STRIEN, One-parameter families of smooth interval maps, density of hyperbolicity and robust chaos, Proc. Amer. Math. Soc. 138 (2010), 4443-4446.

[56] P. Thieullen, C. Tresser, L.-S. Young, Positive Lyapunov exponent for generic one-parameter families of unimodal maps, J. d'Anal. Math. 64 (1994), 121-172.

[57] M. TsujII, Positive Lyapunov exponents in families of one-dimensional dynamical systems, Invent. Math. 111 (1993), 113-137.

[58] M. TsujII, On continuity of Bowen-Ruelle-Sinai measures in families of onedimensional maps, Comm. Math. Phys. 177 (1996), 1-11.

[59] M. Viana, Stochastic dynamics of deterministic systems, 1997, 21 Colóquio Brasileiro de Matemática, IMPA, Rio de Janeiro (1997), available on http://w3.impa.br/ viana (under Lecture Notes).

[60] L. WANG, Topological and metrical conditions for Collet-Eckmann unimodal maps, Acta Math. Appl. Sinica (English Ser.) 17 (2001), 350-360.

[61] L.-S. Young, Decay of correlations for certain quadratic maps, Comm. Math. Phys. 146 (1992), 123-138. 
[62] L.-S. Young, Statistical properties of dynamical systems with some hyperbolicity, Ann. of Math. 147 (1998), 585-650.

[63] L.-S. Young, What are SRB measures, and which dynamical systems have them? Dedicated to David Ruelle and Yasha Sinai on the occasion of their 65th birthday, J. Statist. Phys. 108 (2002), 733-754.

(Manuscrit reçu le 23 novembre 2010; accepté, après révision, le 26 juin 2012.)

\author{
Viviane BALADI \\ D.M.A., UMR CNRS 8553 \\ École Normale Supérieure \\ 45 rue d'Ulm \\ 75005 Paris, France \\ Department of Mathematical Sciences \\ Copenhagen University \\ Universitetspark 5 \\ 2100 Copenhagen, Denmark \\ E-mail: baladi@math.ku.dk \\ Daniel SMANiA \\ Departamento de Matemática \\ ICMC-USP \\ Caixa Postal 668 \\ São Carlos-SP, CEP 13560-970 \\ São Carlos-SP, Brazil \\ E-mail: smania@icmc.usp.br
}

